\title{
Reactions of Cyclopropanone Acetals with Alkyl Azides: Carbonyl Addition versus Ring-Opening Pathways
}

\section{Scott Grecian, ${ }^{\text {a }}$ Pankaj Desai, ${ }^{\text {a }}$ Craig Mossman, ${ }^{\text {a Jennifer L. Poutsma, }}$ and Jeffrey Aubéa,*}

\section{Supporting Information}

\section{Table of Contents}

\begin{tabular}{rrc}
\hline Experimental Section & Page \\
\hline & $\begin{array}{r}\text { p-lactams and ethyl carbamates } \\
\text { preparation of substituted } \\
\text { cyclopropanones }\end{array}$ & $S 6$ \\
& $\begin{array}{r}\text { products resulting from reactions } \\
\text { of substituted cyclopropanones } \\
\text { and alkyl azides }\end{array}$ & $S 9$ \\
Molecular modeling data & $\begin{array}{r}\text { cartesian coordinates and } \\
\text { energies for oxyallyl cations }\end{array}$ & $S 19$ \\
Depictions of X-ray structures & $\begin{array}{r}\text { ball and stick models of 5c and } \\
\mathbf{9 g}\end{array}$ & $S 24$ \\
References for supporting information & $S 24$ \\
${ }^{1} \mathbf{H}$ and ${ }^{13} \mathbf{C}$ NMR spectra & $S 25$ \\
\hline
\end{tabular}

\section{Corresponding author:}

Professor Jeffrey Aubé

Department of Medicinal Chemistry

1251 Wescoe Hall Drive, Room 4070

Malott Hall

University of Kansas

Lawrence, Kansas 66045-7582

Tel 785.864.4496

Fax 785.864.5326

E-mail: jaube@ku.edu 


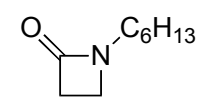

$N$-Hexyl-azetidin-2-one (2b). Clear oil (90 mg, 58\%). $\mathrm{R}_{f}=0.10(20 \%$ EtOAc/hex); IR (neat) 2900, $1740 \mathrm{~cm}^{-1} ;{ }^{1} \mathrm{H}$ NMR (400 MHz, $\left.\mathrm{CDCl}_{3}\right) \delta$ 3.15-3.25 (m, 4H), $2.91(\mathrm{t}, J=6.9 \mathrm{~Hz}, 2 \mathrm{H}), 1.38(\mathrm{~m}, 2 \mathrm{H}), 1.15(\mathrm{br} \mathrm{s}, 6 \mathrm{H}), 0.89$ (br t, $J=6.7 \mathrm{~Hz}, 3 \mathrm{H})$; ${ }^{13} \mathrm{C}$ NMR (400 MHz, $\left.\mathrm{CDCl}_{3}\right) \delta$ 167.2, 41.5, 38.4, 36.1, 31.0, 27.2, 25.3, 22.2, 13.7; CIMS $\mathrm{m} / \mathrm{z}$ (relative intensity) $156\left(\mathrm{MH}^{+}, 100\right), 126,114$; HRMS calcd for $\mathrm{C}_{9} \mathrm{H}_{18} \mathrm{NO}: 156.1388$, found: 156.1395 .

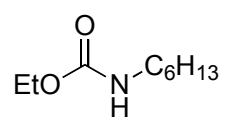

$N$-Hexyl carbamic acid ethyl ester (3b). Clear oil (40\%). Known compound. ${ }^{1}$

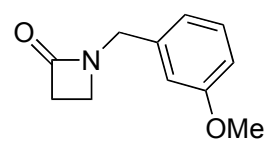

1-(3'-Methoxyphenylmethyl)azetidin-2-one (2c). Pale yellow oil (36\%). $\mathrm{R}_{f}=$ 0.10 (20\% EtOAc/hex); IR (neat) 2961, 1742, $1602 \mathrm{~cm}^{-1} ;{ }^{1} \mathrm{H}$ NMR (400 MHz, $\left.\mathrm{CDCl}_{3}\right) \delta$ $7.26(\mathrm{t}, J=7.9 \mathrm{~Hz}, 1 \mathrm{H}), 6.82(\mathrm{~d}, J=7.9 \mathrm{~Hz}, 2 \mathrm{H}), 6.78(\mathrm{~s}, 1 \mathrm{H}), 4.34(\mathrm{~s}, 2 \mathrm{H}), 3.79(\mathrm{~s}, 3 \mathrm{H})$, $3.14(\mathrm{t}, J=4.1 \mathrm{~Hz}, 2 \mathrm{H}), 2.94(\mathrm{t}, J=4.1 \mathrm{~Hz}, 2 \mathrm{H}) ;{ }^{13} \mathrm{C} \mathrm{NMR}\left(400 \mathrm{MHz}, \mathrm{CDCl}_{3}\right) \delta 167.9$, 160.3, 137.6, 130.2, 120.7, 114.1, 113.4, 55.6, 46.5, 39.0, 37.2; CIMS m/z (relative intensity) $192\left(\mathrm{MH}^{+}, 100\right), 121$ (19); HRMS calcd for $\mathrm{C}_{11} \mathrm{H}_{14} \mathrm{NO}_{2}$ : 192.1024, found: 192.1007. 


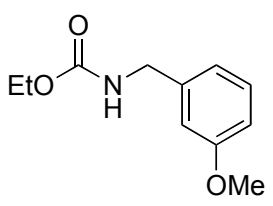

(3-Methoxyphenylmethyl)carbamic acid ethyl ester (3c). Pale yellow oil $(60 \%) . \mathrm{R}_{f}=0.25\left(20 \%\right.$ EtOAc/hex); IR (neat) 3340, 2981, $1709 \mathrm{~cm}^{-1} ;{ }^{1} \mathrm{H}$ NMR (400 $\left.\mathrm{MHz}, \mathrm{CDCl}_{3}\right) \delta 7.22(\mathrm{t}, J=7.8 \mathrm{~Hz}, 1 \mathrm{H}), 6.81(\mathrm{~m}, 3 \mathrm{H}), 5.25$ (br s, 1H), 4.30 (br d, $J=$ $5.8 \mathrm{~Hz}, 2 \mathrm{H}), 4.11(\mathrm{q}, J=6.9 \mathrm{~Hz}, 2 \mathrm{H}), 3.77(\mathrm{~s}, 3 \mathrm{H}), 1.23(\mathrm{t}, J=7.1 \mathrm{~Hz}, 3 \mathrm{H}) ;{ }^{13} \mathrm{C} \mathrm{NMR}$ $\left(400 \mathrm{MHz}, \mathrm{CDCl}_{3}\right) \delta 160.2,157.2,140.8,130.0,120.1,113.4,113.2,61.3,55.6,45.3$, 15.0; CIMS m/z (relative intensity) $210\left(\mathrm{M}^{+}, 100\right), 163$ (16), 136 (23), 121 (10); HRMS calcd for $\mathrm{C}_{11} \mathrm{H}_{16} \mathrm{NO}_{3}: 210.1130$, found: 210.1135 .

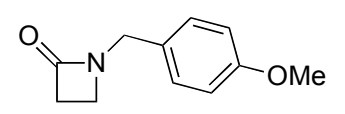

1-(4'-Methoxyphenylmethyl)azetidin-2-one (2d). Clear oil (27\%). $\mathrm{R}_{f}=0.10$ (20\% EtOAc/hex); IR (neat) 2960, 1746, $\left.1510 \mathrm{~cm}^{-1} ;{ }^{1} \mathrm{H} \mathrm{NMR} \mathrm{(400} \mathrm{MHz,} \mathrm{CDCl}_{3}\right) \delta 7.17$ $(\mathrm{d}, J=8.6 \mathrm{~Hz}, 2 \mathrm{H}), 6.87(\mathrm{~d}, J=8.6 \mathrm{~Hz}, 2 \mathrm{H}), 4.31(\mathrm{~s}, 2 \mathrm{H}), 3.80(\mathrm{~s}, 3 \mathrm{H}), 3.11(\mathrm{t}, J=4.1$ $\mathrm{Hz}, 2 \mathrm{H}), 2.92(\mathrm{t}, J=4.1 \mathrm{~Hz}, 2 \mathrm{H}) ;{ }^{13} \mathrm{C} \mathrm{NMR}\left(400 \mathrm{MHz}, \mathrm{CDCl}_{3}\right) \delta 167.9,159.5,129.9$, 128.1, 114.5, 55.7, 46.0, 38.7, 37.1; CIMS m/z (relative intensity) $192\left(\mathrm{MH}^{+}, 100\right), 121$ (40); HRMS calcd for $\mathrm{C}_{11} \mathrm{H}_{14} \mathrm{NO}_{2}$ : 192.1024, found: 192.1016 .

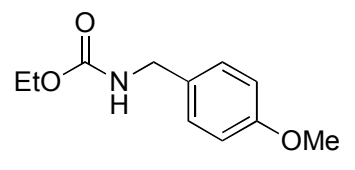

(4-Methoxyphenylmethyl)carbamic acid ethyl ester (3d). Off-white solid $(35 \%) . \mathrm{R}_{f}=0.20(20 \%$ EtOAc/hex $) ; \operatorname{mp~} 37-39^{\circ} \mathrm{C}$; IR $\left(\mathrm{CH}_{2} \mathrm{Cl}_{2}\right) 3340,2985,1706, \mathrm{~cm}^{-1}$; 
${ }^{1} \mathrm{H}$ NMR (400 MHz, $\left.\mathrm{CDCl}_{3}\right) \delta 7.19(\mathrm{~d}, J=8.4 \mathrm{~Hz}, 2 \mathrm{H}), 6.84(\mathrm{~d}, J=8.4 \mathrm{~Hz}, 2 \mathrm{H}), 5.11$ (br s, $1 \mathrm{H}), 4.26(\mathrm{~d}, J=5.7 \mathrm{~Hz}, 2 \mathrm{H}), 4.11(\mathrm{q}, J=7.0 \mathrm{~Hz}, 2 \mathrm{H}), 3.77(\mathrm{~s}, 3 \mathrm{H}) ;{ }^{13} \mathrm{C} \mathrm{NMR}$ (400 MHz, $\left.\mathrm{CDCl}_{3}\right) \delta 159.3,157.1,131.2,129.3,114.4,61.2,55.7,44.8,15.0 ; \mathrm{CIMS} \mathrm{m} / \mathrm{z}$ (relative intensity) $210\left(\mathrm{MH}^{+}, 81\right), 136$ (43), 121 (100); HRMS calcd for $\mathrm{C}_{11} \mathrm{H}_{16} \mathrm{NO}_{3}$ : 210.1135, found: 210.1120 .

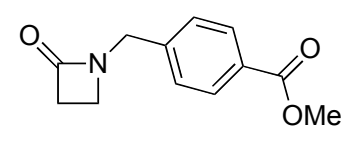

1-(4'-Carbomethoxyphenylmethyl)azetidin-2-one (2e). Off-white solid (38\%). $\mathrm{R}_{f}=0.10(20 \%$ EtOAc/hex $) ; \mathrm{mp} 52-53{ }^{\circ} \mathrm{C} ; \mathrm{IR}\left(\mathrm{CH}_{2} \mathrm{Cl}_{2}\right) 2956,1757,1721 \mathrm{~cm}^{-1} ;{ }^{1} \mathrm{H}$ NMR $\left(400 \mathrm{MHz}, \mathrm{CDCl}_{3}\right) \delta 8.02(\mathrm{~d}, J=8.2 \mathrm{~Hz}, 2 \mathrm{H}), 7.32(\mathrm{~d}, J=8.2 \mathrm{~Hz}, 2 \mathrm{H}), 4.44(\mathrm{~s}, 2 \mathrm{H})$, $3.92(\mathrm{~s}, 3 \mathrm{H}), 3.18(\mathrm{t}, J=4.1 \mathrm{~Hz}, 2 \mathrm{H}), 3.00(\mathrm{t}, J=4.1 \mathrm{~Hz}, 2 \mathrm{H}) ;{ }^{13} \mathrm{C}$ NMR $(400 \mathrm{MHz}$, $\left.\mathrm{CDCl}_{3}\right) \delta 168.0,167.0,141.3,130.5,130.0,128.4,52.5,46.3,39.2,37.5 ; \mathrm{CIMS} \mathrm{m} / \mathrm{z}$ (relative intensity) $220\left(\mathrm{MH}^{+}, 100\right), 149$ (12); HRMS calcd for $\mathrm{C}_{12} \mathrm{H}_{14} \mathrm{NO}_{3}: 220.0973$, found: 220.0967 .

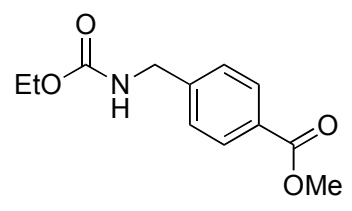

(4'-Carbomethoxyphenylmethyl)carbamic acid ethyl ester (3e). Off-white solid (43\%). $\mathrm{R}_{f}=0.25$ (20\% EtOAc/hex); mp 61-62 ${ }^{\circ} \mathrm{C}$; IR $\left(\mathrm{CH}_{2} \mathrm{Cl}_{2}\right) 3354,2985,1739$, $1706 \mathrm{~cm}^{-1} ;{ }^{1} \mathrm{H}$ NMR $\left(400 \mathrm{MHz}, \mathrm{CDCl}_{3}\right) \delta 7.95(\mathrm{~d}, J=7.4 \mathrm{~Hz}, 2 \mathrm{H}), 7.32(\mathrm{~d}, J=5.2 \mathrm{~Hz}$, 2H), $5.63($ br s, 1H), $4.37(\mathrm{~d}, J=5.2 \mathrm{~Hz}, 2 \mathrm{H}), 4.11(\mathrm{q}, J=6.9 \mathrm{~Hz}, 2 \mathrm{H}), 3.88(\mathrm{~s}, 3 \mathrm{H})$, $1.22(\mathrm{t}, J=6.8 \mathrm{~Hz}, 3 \mathrm{H}) ;{ }^{13} \mathrm{C} \mathrm{NMR}\left(400 \mathrm{MHz}, \mathrm{CDCl}_{3}\right) \delta 167.2,157.3,144.6,130.2$, 
129.4, 127.5, 61.4, 52.4, 44.9, 15.0; CIMS m/z (relative intensity) $238\left(\mathrm{MH}^{+}, 100\right), 164$ (29), 132 (26); HRMS calcd for $\mathrm{C}_{12} \mathrm{H}_{16} \mathrm{NO}_{4}$ : 238.0179, found: 238.1083.

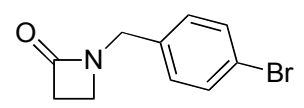

1-(4'-Bromophenylmethyl)azetidin-2-one (2f). Off-white solid (45\%). $\mathrm{R}_{f}=0.10$ (20\% EtOAc/hex); mp 63-64 ${ }^{\circ} \mathrm{C}$; IR $\left(\mathrm{CH}_{2} \mathrm{Cl}_{2}\right)$ 2913, 1733, $1405 \mathrm{~cm}^{-1} ;{ }^{1} \mathrm{H}$ NMR (400 $\left.\mathrm{MHz}, \mathrm{CDCl}_{3}\right) \delta 7.46(\mathrm{~d}, J=8.1 \mathrm{~Hz}, 2 \mathrm{H}), 7.13(\mathrm{~d}, J=8.1 \mathrm{~Hz}, 2 \mathrm{H}), 4.33(\mathrm{~s}, 2 \mathrm{H}), 3.15(\mathrm{t}$, $J=4.1 \mathrm{~Hz}, 2 \mathrm{H}), 2.96(\mathrm{t}, J=4.1 \mathrm{~Hz}, 2 \mathrm{H}) ;{ }^{13} \mathrm{C} \mathrm{NMR}\left(400 \mathrm{MHz}, \mathrm{CDCl}_{3}\right) \delta 168.0,135.2$, 132.3, 130.2, 122.0, 46.0, 39.0, 37.4; CIMS m/z (relative intensity) $240\left(\mathrm{MH}^{+}, 100\right), 171$ (15), 160 (15), 132 (22), 89 (16); HRMS calcd for $\mathrm{C}_{10} \mathrm{H}_{11} \mathrm{NOBr}$ : 240.0024, found: 240.0026 .

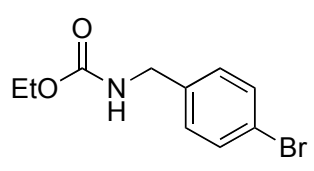

(4-Bromophenyl methyl)-carbamic acid ethyl ester (3f). Off-white solid (34\%). $\mathrm{R}_{f}=0.30$ (20\% EtOAc/hex); mp 70-71 ${ }^{\circ} \mathrm{C} ; \mathrm{IR}\left(\mathrm{CH}_{2} \mathrm{Cl}_{2}\right) 3320,2985,1704,1539 \mathrm{~cm}^{-1} ;{ }^{1} \mathrm{H}$ NMR (400 MHz, $\left.\mathrm{CDCl}_{3}\right) \delta 7.39(\mathrm{~d}, J=8.3 \mathrm{~Hz}, 2 \mathrm{H}), 7.11(\mathrm{~d}, J=8.2 \mathrm{~Hz}, 2 \mathrm{H}), 5.50(\mathrm{br}$ s, $1 \mathrm{H}), 4.24(\mathrm{~d}, J=6.1 \mathrm{~Hz}, 2 \mathrm{H}), 4.08(\mathrm{q}, J=7.1 \mathrm{~Hz}, 2 \mathrm{H}), 1.21(\mathrm{t}, J=7.1 \mathrm{~Hz}, 3 \mathrm{H}) ;{ }^{13} \mathrm{C}$ NMR (400 MHz, $\left.\mathrm{CDCl}_{3}\right) \delta 157.2,138.3,132.0,129.5,121.5,61.4,44.6,15.0 ; \mathrm{CIMS} \mathrm{m} / \mathrm{z}$ (relative intensity) $258\left(\mathrm{MH}^{+}, 100\right), 184$ (51); HRMS calcd for $\mathrm{C}_{10} \mathrm{H}_{13} \mathrm{NO}_{2} \mathrm{Br}$ : 258.0129, found: 258.0109. 
Preparation of substituted cyclopropanone silyl acetals. Silyl ketene acetals were prepared according to the method developed by Ainsworth, except triethylsilyl chloride was used instead of trimethylsilyl chloride as a trapping agent. ${ }^{2}$ The crude silyl ketene acetals were directly submitted to Simmons-Smith cyclopropanation conditions developed by Slougui ${ }^{3}$ followed by chromatography $(5 \% /$ EtOAc/hex) to give the indicated triethylsiloxy cyclopropanone acetals.

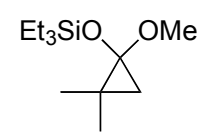

( \pm )-Triethyl(1-methoxy-2,2-dimethylcyclopropoxy)silane (4). Triethyl-(1-

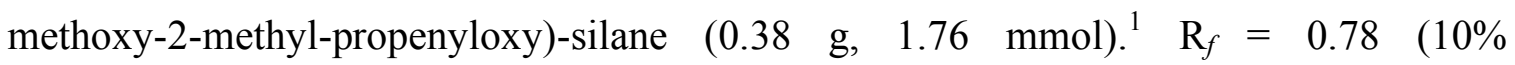
EtOAc/hex); ${ }^{1} \mathrm{H}$ NMR (400 MHz, $\mathrm{CDCl}_{3} \delta 3.52$ (s, 3H), 1.56 (s, 3H), 1.54 (s, 3H), 1.00 $(\mathrm{m}, 9 \mathrm{H}), 0.70(\mathrm{~m}, 6 \mathrm{H})$. The crude material was then subjected to Simmons-Smith cyclopropanation conditions ${ }^{2}$ to give the title compound as a clear oil (180 mg, 44\%). $\mathrm{R}_{f}$ $=0.34$ (hex); IR (neat) 2950, 1470, $\mathrm{cm}^{-1} ;{ }^{1} \mathrm{H}$ NMR $\left(400 \mathrm{MHz}, \mathrm{CDCl}_{3}\right) \delta 3.36(\mathrm{~s}, 3 \mathrm{H})$, $1.15(\mathrm{~s}, 3 \mathrm{H}), 1.14(\mathrm{~s}, 3 \mathrm{H}), 1.00$ (t, $J=7.9 \mathrm{~Hz}, 9 \mathrm{H}), 0.70$ (q, $J=7.9 \mathrm{~Hz}, 6 \mathrm{H}) 0.56$ (dd, $J=$ $5.3 \mathrm{~Hz}, 2 \mathrm{H}) ;{ }^{13} \mathrm{C} \mathrm{NMR}\left(100 \mathrm{MHz}, \mathrm{CDCl}_{3}\right) \delta 92.9,54.3,26.3,24.2,21.5,21.0,7.2,5.6$; MS (CI) m/z $\left(M^{+}\right)$, 230; HRMS calcd for $\mathrm{C}_{12} \mathrm{H}_{30} \mathrm{NO}_{2} \mathrm{Si}\left(\mathrm{M}^{+}+\mathrm{NH}_{4}\right)$ : 248.2046, found 248.2057. 


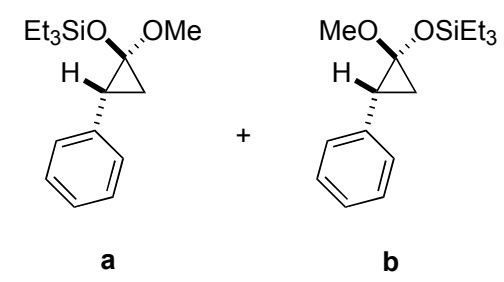

$\left(1 S^{*}, 2 R^{*}\right)$-Triethyl-(1-methoxy-2-phenylcyclopropoxy)silane (7a) and $\left(1 R^{*}, 2 R^{*}\right)$-Triethyl-(1-methoxy-2-phenylcyclopropoxy)silane (7b). A crude mixture of 2.8:1 E- and Z-triethyl-(1-methoxy-2-phenyl-vinyloxy)silane was prepared according to the protocol described by Ainsworth $(4.3 \mathrm{~mL}, 30.0 \mathrm{mmol}) . \mathrm{R}_{f}=0.66$ and $0.70(20 \%$ EtOAc/hex). $\boldsymbol{E}$-isomer: ${ }^{1} \mathrm{H} \mathrm{NMR}\left(500 \mathrm{MHz}, \mathrm{CDCl}_{3}\right) \delta 7.48(\mathrm{~d}, J=7.5 \mathrm{~Hz}, 2 \mathrm{H}), 7.27$ (t, $J$ $=7.8 \mathrm{~Hz}, 3 \mathrm{H}), 4.72(\mathrm{~s}, 1 \mathrm{H}), 3.77(\mathrm{~s}, 3 \mathrm{H}), 1.08(\mathrm{~m}, 9 \mathrm{H}), 0.96(\mathrm{~m}, 6 \mathrm{H}) . \boldsymbol{Z}$-isomer: ${ }^{1} \mathrm{H}$ NMR $\left(500 \mathrm{MHz}, \mathrm{CDCl}_{3}\right) \delta 7.48(\mathrm{~d}, J=7.5 \mathrm{~Hz}, 2 \mathrm{H}), 7.05(\mathrm{t}, J=7.4 \mathrm{~Hz}, 3 \mathrm{H}), 4.61(\mathrm{~s}, 1 \mathrm{H}), 3.71$ $(\mathrm{s}, 3 \mathrm{H}), 0.93(\mathrm{~m}, 9 \mathrm{H}), 0.63(\mathrm{~m}, 6 \mathrm{H})$. This mixture was directly subjected to cyclopropanation and then chromatography (5\% EtOAc/hex) to give the title compound as a 3:2 diastereomeric mixture (clear oil, $7.4 \mathrm{~g}, 89 \%$ over two steps). $\mathrm{R}_{f}=0.70(20 \%$ EtOAc/hex); IR (neat) 2953, $1614 \mathrm{~cm}^{-1}$; MS (FAB) m/z $278\left(\mathrm{M}^{+}\right)$; HRMS calcd for $\mathrm{C}_{16} \mathrm{H}_{26} \mathrm{O}_{2} \mathrm{Si}: 278.1702$, found 278.1709. (1S*,2R*)-a: ${ }^{1} \mathrm{H}$ NMR (400 MHz, $\left.\mathrm{CDCl}_{3}\right) \delta 7.30$ $(\mathrm{m}, 5 \mathrm{H}), 3.30(\mathrm{~s}, 3 \mathrm{H}), 2.41(\mathrm{~m}, 1 \mathrm{H}), 1.48(\mathrm{~m}, 2 \mathrm{H}), 1.12(\mathrm{t}, J=8.0 \mathrm{~Hz}, 9 \mathrm{H}), 0.85(\mathrm{q}, J=$ $7.7 \mathrm{~Hz}, 6 \mathrm{H}) ;{ }^{13} \mathrm{C} \mathrm{NMR}\left(100 \mathrm{MHz}, \mathrm{CDCl}_{3}\right) \delta 138.3,128.5,127.8,126.1,89.9,54.3,32.1$, 21.5, 7.2, 5.6. $\left(\mathbf{1} \boldsymbol{R}^{*}, \mathbf{2} \boldsymbol{R}^{*}\right)-\mathbf{b}:{ }^{1} \mathrm{H} \mathrm{NMR}\left(400 \mathrm{MHz}, \mathrm{CDCl}_{3}\right.$, diagnostic peaks only) $\delta 3.55$ $(\mathrm{s}, 3 \mathrm{H}), 0.99(\mathrm{t}, J=8.0 \mathrm{~Hz}, 9 \mathrm{H}), 0.67(\mathrm{q}, J=7.7 \mathrm{~Hz}, 6 \mathrm{H}) ;{ }^{13} \mathrm{C} \mathrm{NMR}\left(100 \mathrm{MHz}, \mathrm{CDCl}_{3}\right) \delta$ $138.4,128.6,128.2,126.2,90.2,54.1,30.6,21.6,7.1,5.6$ 


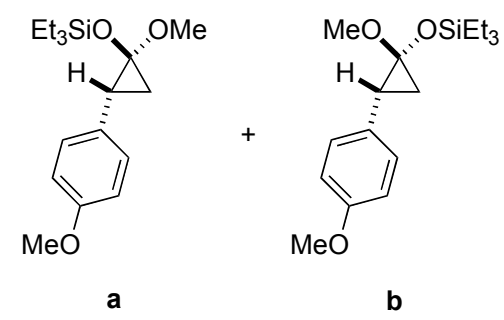

$\left(1 S^{*}, 2 R^{*}\right)$-Triethyl-(1-methoxy-2-(4-methoxyphenyl)cyclopropoxy) silane

(8a) and $\left(1 R^{*}, 2 R^{*}\right)$-Triethyl-(1-methoxy-2-(4-methoxyphenyl) cyclopropoxy)silane

(8b). A mixture of 3:2 E- and Z-triethyl-(1-methoxy-2-(4-methoxy-phenyl)vinyloxy)silane was prepared $(5.2 \mathrm{~mL}, 20.0 \mathrm{mmol}) . \mathrm{R}_{f}=0.29$ and 0.37 (5\% EtOAc/hex).

E-isomer: ${ }^{1} \mathrm{H}$ NMR $\left(400 \mathrm{MHz}, \mathrm{CDCl}_{3}\right) \delta 7.36(\mathrm{~d}, J=8.8 \mathrm{~Hz}, 2 \mathrm{H}), 6.84(\mathrm{~d}, J=8.8 \mathrm{~Hz}$, 2H), 4.69 (s, 1H), $3.82(\mathrm{~s}, 3 \mathrm{H}), 3.74(\mathrm{~s}, 3 \mathrm{H}), 1.01(\mathrm{~m}, 9 \mathrm{H}), 0.97(\mathrm{~m}, 6 \mathrm{H}) . \boldsymbol{Z}$-isomer: ${ }^{1} \mathrm{H}$ NMR (400 MHz, $\left.\mathrm{CDCl}_{3}\right) \delta 7.36(\mathrm{~d}, J=7.5 \mathrm{~Hz}, 2 \mathrm{H}), 6.85(\mathrm{~d}, J=8.8 \mathrm{~Hz}, 2 \mathrm{H}), 3.84(\mathrm{~s}$, $3 \mathrm{H}), 3.74(\mathrm{~s}, 3 \mathrm{H}), 3.69(\mathrm{~s}, 1 \mathrm{H}), 1.01(\mathrm{~m}, 9 \mathrm{H}), 0.64(\mathrm{~m}, 6 \mathrm{H})$. This material was subjected to cyclopropanation and chromatography (5\% EtOAc/hex) to give the title compound as a $3: 2$ diastereomeric mixture (clear oil, $5.03 \mathrm{~g}, 82 \%$ over two steps). $\mathrm{R}_{f}=0.60(20 \%$ EtOAc/hex); IR (neat) 2946, 1614, 1520, cm ${ }^{-1}$; MS (FAB) m/z $308\left(\mathrm{M}^{+}\right)$; HRMS calcd for $\mathrm{C}_{17} \mathrm{H}_{28} \mathrm{O}_{2} \mathrm{Si}: 308.1808$, found 308.1805. (1S*,2R*)-8a: ${ }^{1} \mathrm{H}$ NMR (500 MHz, $\left.\mathrm{CDCl}_{3}\right) \delta$ $7.17(\mathrm{~d}, J=2.8 \mathrm{~Hz}, 2 \mathrm{H}), 6.90(\mathrm{~d}, J=2.3 \mathrm{~Hz}, 2 \mathrm{H}), 3.826(\mathrm{~s}, 3 \mathrm{H}), 3.28(\mathrm{~s}, 3 \mathrm{H}), 2.36$ (dd, $J$ $=7.0,5.7 \mathrm{~Hz}, 1 \mathrm{H}), 1.44(\mathrm{dd}, J=6.5,4.8 \mathrm{~Hz}, 1 \mathrm{H}), 1.33(\mathrm{t}, J=5.4 \mathrm{~Hz}, 1 \mathrm{H}), 1.09(\mathrm{t}, J=6.4$ $\mathrm{Hz}, 9 \mathrm{H}), 0.80(\mathrm{q}, J=6.4 \mathrm{~Hz}, 6 \mathrm{H}) ;{ }^{13} \mathrm{C} \mathrm{NMR}\left(125 \mathrm{MHz}, \mathrm{CDCl}_{3}\right) \delta 157.8,129.7,128.2$, $113.5,89.1,55.0,53.7,30.8,20.6,6.7,5.1 .\left(1 \boldsymbol{R}^{*}, 2 \boldsymbol{R} *\right)-8 \mathbf{b}:{ }^{1} \mathrm{H}$ NMR $\left(500 \mathrm{MHz}, \mathrm{CDCl}_{3}\right) \delta$ $7.19(\mathrm{~d}, J=2.8 \mathrm{~Hz}, 2 \mathrm{H}), 6.88(\mathrm{~d}, J=2.3 \mathrm{~Hz}, 2 \mathrm{H}), 3.830(\mathrm{~s}, 3 \mathrm{H}), 3.51(\mathrm{~s}, 3 \mathrm{H}), 2.30$ (dd, $J$ $=7.1,5.6 \mathrm{~Hz}, 1 \mathrm{H}), 1.54(\mathrm{dd}, J=6.6,4.7 \mathrm{~Hz}, 1 \mathrm{H}), 1.21(\mathrm{t}, J=5.2 \mathrm{~Hz}, 1 \mathrm{H}), 0.97(\mathrm{t}, J=6.4$ $\mathrm{Hz}, 9 \mathrm{H}), 0.64(\mathrm{q}, J=6.4 \mathrm{~Hz}, 6 \mathrm{H}) ;{ }^{13} \mathrm{C} \mathrm{NMR}\left(125 \mathrm{MHz}, \mathrm{CDCl}_{3}\right) \delta 157.8,129.7,128.0$, $113.2,89.6,55.1,53.5,29.1,21.0,6.6,5.1$. 


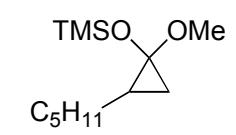

( \pm )-Trimethyl(1-methoxy-2-pentylcyclopropoxy)silane (11). Known

compound. $^{3}$

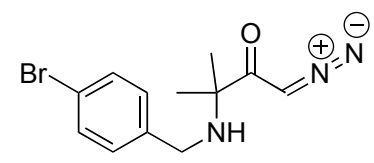

3-(4'-Bromophenyl)amino-1-diazo-3-methyl-butan-2-one (5b). Yellow oil (41\%): $\mathrm{R}_{f}=0.05\left(20 \%\right.$ EtOAc/hex); IR (neat) $3323,3123,2099,1633, \mathrm{~cm}^{-1} ;{ }^{1} \mathrm{H}$ NMR (400 MHz, $\left.\mathrm{CDCl}_{3}\right) \delta 7.44$ (d, $\left.J=8.3 \mathrm{~Hz}, 2 \mathrm{H}\right), 7.23$ (d, $\left.J=8.3 \mathrm{~Hz}, 2 \mathrm{H}\right), 5.88$ (br s, $\left.1 \mathrm{H}\right)$, $3.60(\mathrm{~s}, 2 \mathrm{H}), 1.64$ (br s, 1H), $1.33(\mathrm{~s}, 6 \mathrm{H}) ;{ }^{13} \mathrm{C}$ NMR $\left(400 \mathrm{MHz}, \mathrm{CDCl}_{3}\right) \delta 200.9,139.8$, 131.9, 130.1, 121.2, 62.6, 52.4, 48.0, 25.8; CIMS m/z (relative intensity) 298 (M+2, 55), 296 (58), 270 (97), 268 (100); HRMS calcd for $\mathrm{C}_{12} \mathrm{H}_{14} \mathrm{BrN}_{3} \mathrm{O}$ : 296.0398, found: 296.0370.

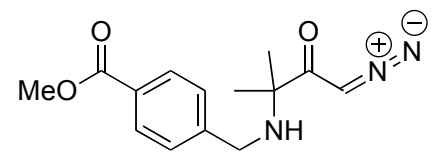

4-(3'-Diazo-1',1'-dimethyl-2'-oxo-propylamino)benzoic acid methyl ester (5c).

Yellow solid (46\%): $\mathrm{R}_{f}=0.05$ (20\% EtOAc/hex); mp 88-89 ${ }^{\circ} \mathrm{C}$; IR (neat) 2099, 1707, $1633 \mathrm{~cm}^{-1} ;{ }^{1} \mathrm{H}$ NMR $\left(400 \mathrm{MHz}, \mathrm{CDCl}_{3}\right) \delta 8.00(\mathrm{~d}, J=8.1 \mathrm{~Hz}, 2 \mathrm{H}), 7.43(\mathrm{~d}, J=8.1 \mathrm{~Hz}$, 2H), 5.90 (br s, 1H), $3.92(\mathrm{~s}, 3 \mathrm{H}), 3.72(\mathrm{~s}, 2 \mathrm{H}), 1.66$ (br s, $1 \mathrm{H}), 1.35$ (s, 6H); ${ }^{13} \mathrm{C}$ NMR $\left(400 \mathrm{MHz}, \mathrm{CDCl}_{3}\right) \delta \quad 200.8,167.4,146.1,130.2,129.3,128.2,62.7,52.5,52.4,48.3$, 25.8; CIMS m/z (relative intensity) $276\left(\mathrm{MH}^{+}, 27\right), 248$ (100); HRMS calcd for 
$\mathrm{C}_{14} \mathrm{H}_{18} \mathrm{~N}_{3} \mathrm{O}_{3}$ : 276.1348, found: 276.1344. This compound was submitted to X-ray crystallography; these data have appeared previously. ${ }^{4}$

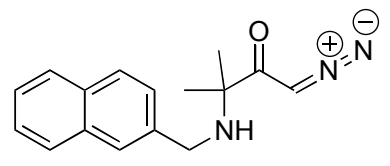

1-Diazo-3-methyl-3-(naphthalen-2'-yl)amino-butan-2-one (5d). Yellow oil (38\%): $\mathrm{R}_{f}=0.07$ (20\% EtOAc/hex); IR (neat) 3323, 3119, 2099, $1633 \mathrm{~cm}^{-1} ;{ }^{1} \mathrm{H}$ NMR (400 MHz, $\left.\mathrm{CDCl}_{3}\right) \delta$ 7.80-7.83 (m, 4H), 7.45-7.48 (m, 3H), 5.99 (br s, 1H), $3.82(\mathrm{~s}, 2 \mathrm{H})$, 1.66 (br s, $1 \mathrm{H}), 1.38(\mathrm{~s}, 6 \mathrm{H}) ;{ }^{13} \mathrm{C} \mathrm{NMR}\left(400 \mathrm{MHz}, \mathrm{CDCl}_{3}\right) \delta$ 201.2, 138.2, 133.9, 133.1, $128.5,128.1,126.9,126.6,126.5,126.1,62.7,52.3,48.8,25.9 ;$ CIMS m/z (relative intensity) $268\left(\mathrm{MH}^{+}, 74\right), 198$ (100); HRMS calcd for $\mathrm{C}_{16} \mathrm{H}_{17} \mathrm{~N}_{3} \mathrm{O}: 268.1450$, found: 268.1445 .

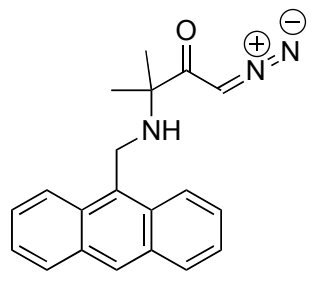

3-(Anthracen-9'-yl)methyl-amino-1-diazo-3-methyl-butan-2-one (5e). Brown oil (54\%): $\mathrm{R}_{f}=0.07$ (20\% EtOAc/hex); IR (neat) 3323, 3057, 2099, $1628 \mathrm{~cm}^{-1} ;{ }^{1} \mathrm{H}$ NMR $\left(400 \mathrm{MHz}, \mathrm{CDCl}_{3}\right) \delta 8.41(\mathrm{~s}, 1 \mathrm{H}), 8.33(\mathrm{~d}, J=8.9 \mathrm{~Hz}, 2 \mathrm{H}), 8.00(\mathrm{~d}, J=8.3 \mathrm{~Hz}, 2 \mathrm{H})$, 7.54-7.58 (m, 2H), 7.45-7.49 (m, 2H), 5.91 (br s, 1H), 4.59 (s, 2H), 1.70 (br s, 1H), 1.50 (s, 6H); ${ }^{13} \mathrm{C} \mathrm{NMR}\left(400 \mathrm{MHz}, \mathrm{CDCl}_{3}\right) \delta$ 201.0, 132.0, 131.5, 130.6, 129.6, 127.8, 126.6, 125.4, 124.4, 62.8, 52.6, 40.3, 25.7; CIMS m/z (relative intensity) 191 (100); HRMS calcd for $\mathrm{C}_{20} \mathrm{H}_{20} \mathrm{~N}_{3} \mathrm{O}: 318.1606$, found: 318.1577 . 


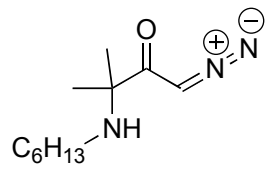

1-Diazo-3-hexylamino-3-methyl-butan-2-one (5f). Yellow oil (44\%): $\mathrm{R}_{f}=0.05$ (20\% EtOAc/hex); IR (neat) 3326, 3122, 2099, $1633 \mathrm{~cm}^{-1} ;{ }^{1} \mathrm{H}$ NMR (400 MHz, $\left.\mathrm{CDCl}_{3}\right) \delta$ $5.86($ br s, $1 \mathrm{H}), 2.44(\mathrm{t}, J=7.0 \mathrm{~Hz}, 2 \mathrm{H}), 1.29-1.43(\mathrm{~m}, 9 \mathrm{H}), 1.25(\mathrm{~s}, 6 \mathrm{H}), 0.89(\mathrm{t}, J=6.7$ $\mathrm{Hz}, 3 \mathrm{H}) ;{ }^{13} \mathrm{C} \mathrm{NMR}\left(400 \mathrm{MHz}, \mathrm{CDCl}_{3}\right) \delta 201.8,62.3,52.0,44.3,32.2,31.3,27.4,25.7$, 23.0, 14.4; CIMS m/z (relative intensity) $212\left(\mathrm{MH}^{+}, 100\right), 184$ (75), 142 (72); HRMS calcd for $\mathrm{C}_{11} \mathrm{H}_{22} \mathrm{~N}_{3} \mathrm{O}: 212.1763$, found: 212.1742 .

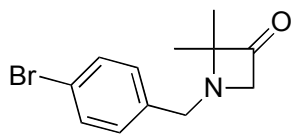

1-(4'-Bromobenzyl)-2,2-dimethyl-azetidin-3-one (6b). Yellow oil (87\%): $\mathrm{R}_{f}=$ 0.15 (20\% EtOAc/hex); IR (neat) 2971, $1803 \mathrm{~cm}^{-1} ;{ }^{1} \mathrm{H}$ NMR (400 MHz, $\left.\mathrm{CDCl}_{3}\right) \delta 7.44$ $(\mathrm{d}, J=8.3 \mathrm{~Hz}, 2 \mathrm{H}), 7.26(\mathrm{~d}, J=8.3 \mathrm{~Hz}, 2 \mathrm{H}), 4.02(\mathrm{~s}, 2 \mathrm{H}), 3.79(\mathrm{~s}, 2 \mathrm{H}), 1.28(\mathrm{~s}, 6 \mathrm{H}) ;{ }^{13} \mathrm{C}$ NMR (400 MHz, $\left.\mathrm{CDCl}_{3}\right) \delta$ 208.6, 138.3, 131.9, 130.4, 121.3, 84.0, 70.9, 54.7, 20.4; CIMS m/z (relative intensity) $270(\mathrm{M}+2,27), 268$ (29), 70 (100); HRMS calcd for $\mathrm{C}_{12} \mathrm{H}_{14} \mathrm{BrNO}$ : 267.0259, found: 267.0238.

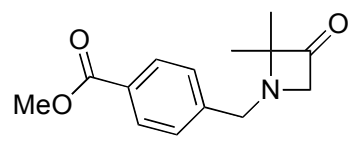

4-(2',2'-Dimethyl-3'-oxo-azetidin-1'-yl)methylbenzoic acid methyl ester (6c). Yellow oil (77\%): $\mathrm{R}_{f}=0.16\left(20 \%\right.$ EtOAc/hex); IR (neat) $2973,1804,1721 \mathrm{~cm}^{-1} ;{ }^{1} \mathrm{H}$ NMR (400 MHz, $\left.\mathrm{CDCl}_{3}\right) \delta 8.00(\mathrm{~d}, J=8.3 \mathrm{~Hz}, 2 \mathrm{H}), 7.47(\mathrm{~d}, J=8.1 \mathrm{~Hz}, 2 \mathrm{H}), 4.05(\mathrm{~s}$, 
2H), $3.91(\mathrm{~s}, 3 \mathrm{H}), 3.90(\mathrm{~s}, 2 \mathrm{H}), 1.30(\mathrm{~s}, 6 \mathrm{H}) ;{ }^{13} \mathrm{C} \mathrm{NMR}\left(400 \mathrm{MHz}, \mathrm{CDCl}_{3}\right) \delta$ 208.5, $167.3,144.7,130.1,129.5,128.6,84.2,71.0,55.1,52.5,20.4 ;$ CIMS m/z (relative intensity) $248\left(\mathrm{MH}^{+}, 100\right), 70$ (66); HRMS calcd for $\mathrm{C}_{14} \mathrm{H}_{18} \mathrm{NO}_{3}$ : 248.1287, found: 248.1276.

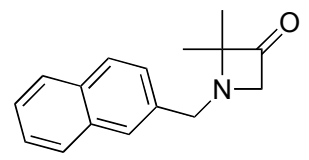

2,2-Dimethyl-1-(naphthalen-2'-yl)methyl-azetidin-3-one (6d). Off-white solid (95\%): $\mathrm{R}_{f}=0.16(20 \% \mathrm{EtOAc} / \mathrm{hex}) ; \mathrm{mp} 81-82{ }^{\circ} \mathrm{C}$; IR (neat) 2970, $1804 \mathrm{~cm}^{-1} ;{ }^{1} \mathrm{H} \mathrm{NMR}$ $\left(400 \mathrm{MHz}, \mathrm{CDCl}_{3}\right) \delta$ 7.80-7.84 (m, 4H), 7.53-7.56 (dd, $\left.J=1.5,8.4 \mathrm{~Hz}, 1 \mathrm{H}\right), 7.45-7.48(\mathrm{~m}$, 2H), 4.08 (s, 2H), $4.00(\mathrm{~s}, 2 \mathrm{H}), 1.33$ (s, 6H); $\left.{ }^{13} \mathrm{C} \mathrm{NMR} \mathrm{(400} \mathrm{MHz,} \mathrm{CDCl}_{3}\right) \delta$ 209.1, 136.8, $133.8,133.2,128.5,128.1,127.2,127.1,126.5,126.1 ;$ CIMS m/z (relative intensity) 240 $\left(\mathrm{MH}^{+}, 100\right), 141$ (25), 70 (72); HRMS calcd for $\mathrm{C}_{16} \mathrm{H}_{18} \mathrm{NO}$ : 240.1388, found: 240.1407 .

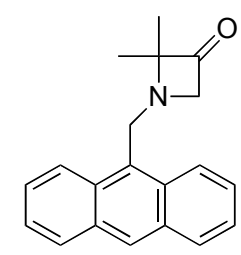

1-(Anthracen-9'-yl)methyl-2,2-dimethyl-azetidin-3-one (6e). Yellow oil (100\%): $\mathrm{R}_{f}=0.18(20 \% \mathrm{EtOAc} / \mathrm{hex}) ; \mathrm{IR}$ (neat) 2966, $1800 \mathrm{~cm}^{-1} ;{ }^{1} \mathrm{H}$ NMR (400 MHz, $\left.\mathrm{CDCl}_{3}\right) \delta 8.49(\mathrm{~d}, J=8.8 \mathrm{~Hz}, 2 \mathrm{H}), 8.42(\mathrm{~s}, 1 \mathrm{H}), 8.00(\mathrm{~d}, J=8.3 \mathrm{~Hz}, 2 \mathrm{H}), 7.51-7.55(\mathrm{~m}$, 2H), 7.44-7.48 (m, 2H), $4.80(\mathrm{~s}, 2 \mathrm{H}), 3.96(\mathrm{~s}, 2 \mathrm{H}), 1.48(\mathrm{~s}, 6 \mathrm{H}) ;{ }^{13} \mathrm{C}$ NMR (400 MHz, $\left.\mathrm{CDCl}_{3}\right) \delta \quad 208.8,134.5,132.0,131.1,129.7,129.6,129.4,128.2,127.6,126.3,126.1$, 
125.3, 124.7, 84.1, 70.2, 45.4, 20.4; CIMS m/z (relative intensity) $290\left(\mathrm{MH}^{+}, 7\right), 209$ (40), $191(100)$.

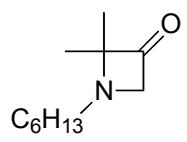

1-Hexyl-2,2-dimethyl-azetidin-3-one (6f). Yellow oil (72\%): $\mathrm{R}_{f}=0.14(20 \%$ EtOAc/hex); IR (neat) 2927, $1806 \mathrm{~cm}^{-1} ;{ }^{1} \mathrm{H}$ NMR (400 MHz, $\left.\mathrm{CDCl}_{3}\right) \delta 4.01(\mathrm{~s}, 2 \mathrm{H}), 2.62$ (t, $J=7.1 \mathrm{~Hz}, 2 \mathrm{H}), 1.31-1.38(\mathrm{~m}, 8 \mathrm{H}), 1.26(\mathrm{~s}, 6 \mathrm{H}), 0.90(\mathrm{t}, J=6.7 \mathrm{~Hz}, 3 \mathrm{H}) ;{ }^{13} \mathrm{C} \mathrm{NMR}$ $\left(400 \mathrm{MHz}, \mathrm{CDCl}_{3}\right) \delta$ 209.3, 83.3, 70.9, 51.4, 32.1, 29.8, 27.5, 23.0, 20.1, 14.4; CIMS m/z (relative intensity) $184\left(\mathrm{MH}^{+}, 100\right), 142(57), 91$ (21); HRMS calcd for $\mathrm{C}_{11} \mathrm{H}_{22} \mathrm{NO}$ : 184.1701, found: 184.1692 .

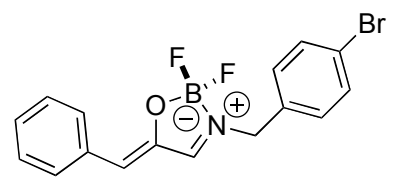

\section{3-(4'-Bromobenzyl)-5-benzylidene-2,2-difluoro-2,5-dihydro-}

[1,2,3]oxaborazole (9b). Off-white solid (57 mg, 40\%). $\mathrm{R}_{f}=0.18$ (20\% EtOAc/hex); mp 161-163 ${ }^{\circ} \mathrm{C}$; IR (KBr pellet) 3440, 1614, $1382 \mathrm{~cm}^{-1} ;{ }^{1} \mathrm{H}$ NMR $\left(500 \mathrm{MHz}, \mathrm{CDCl}_{3} \delta 7.86\right.$ (d, $J=7.1 \mathrm{~Hz}, 2 \mathrm{H}), 7.64(\mathrm{br} \mathrm{s}, 1 \mathrm{H}), 7.61(\mathrm{~d}, J=8.3 \mathrm{~Hz}, 2 \mathrm{H}), 7.37$ (m, 3H), 7.25 (d, $J=$ $8.3 \mathrm{~Hz}, 2 \mathrm{H}, 6.02(\mathrm{~s}, 1 \mathrm{H}), 4.81(\mathrm{~s}, 2 \mathrm{H}) ;{ }^{13} \mathrm{C}$ NMR $\left(125 \mathrm{MHz}, \mathrm{CDCl}_{3}\right) \delta$ 164.0, 149.0, $133.5,132.7,131.4,131.2,130.7,129.6,128.6,123.6,119.6,51.4 ; \mathrm{MS}(\mathrm{FAB}) \mathrm{m} / \mathrm{z}\left(\mathrm{M}^{+}\right)$ 363; HRMS calcd for $\mathrm{C}_{17} \mathrm{H}_{15} \mathrm{BrBF}_{2} \mathrm{NO}_{2}$ : 363.0241 , found 363.0242 . 


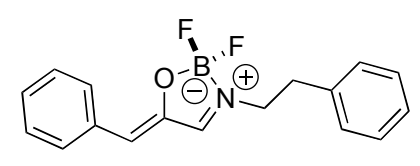

\section{3-Phenylethyl-5-benzylidene-2,2-difluoro-2,5-dihydro-[1,2,3]oxaborazole}

(9c). Light yellow solid (44 mg, 44\%). $\mathrm{R}_{f}=0.11\left(20 \%\right.$ EtOAc/hex); mp 153-156 ${ }^{\circ} \mathrm{C}$; IR (KBr pellet) 3062, $1600 \mathrm{~cm}^{-1} ;{ }^{1} \mathrm{H}$ NMR $\left(500 \mathrm{MHz}, \mathrm{CDCl}_{3} \delta 7.88(\mathrm{~d}, J=8.0 \mathrm{~Hz}, 2 \mathrm{H})\right.$, $7.36(\mathrm{~m}, 5 \mathrm{H}), 7.22(\mathrm{~d}, J=7.8 \mathrm{~Hz}, 2 \mathrm{H}), 5.90(\mathrm{~s}, 1 \mathrm{H}), 4.00(\mathrm{t}, J=6.7 \mathrm{~Hz}, 2 \mathrm{H}), 3.17$ (t, $J=$ $6.7 \mathrm{~Hz}) ;{ }^{13} \mathrm{C}$ NMR $\left(125 \mathrm{MHz}, \mathrm{CDCl}_{3}\right) \delta 164.4,148.7,136.7,133.6,130.6,129.4,129.0$, 128.9, 128.5, 121.2, 118.5, 51.2, 34.8; MS (FAB) m/z $299\left(\mathrm{M}^{+}\right)$, 280; HRMS calcd for $\mathrm{C}_{17} \mathrm{H}_{16} \mathrm{BF}_{2} \mathrm{NO}: 299.1293$, found 299.1287.

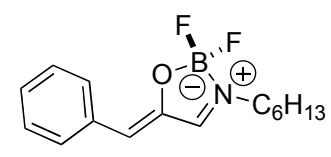

\section{3-Hexyl-5-benzylidene-2,2-difluoro-2,5-dihydro-[1,2,3]oxaborazole}

(9d). White crystals (48 mg, 48\%). $\mathrm{R}_{f}=0.20(20 \% \mathrm{EtOAc} / \mathrm{hex}) ; \mathrm{mp} 108-109{ }^{\circ} \mathrm{C}$; IR (neat) 2917, 1644, 1607, $1076 \mathrm{~cm}^{-1} ;{ }^{1} \mathrm{H}$ NMR $\left(500 \mathrm{MHz}, \mathrm{CDCl}_{3} \delta 7.94(\mathrm{~s}, 1 \mathrm{H}), 7.91\right.$ (d, $J=7.5$ $\mathrm{Hz}, 2 \mathrm{H}), 7.37$ (m, 3H), 6.07 (s, 1H), 1.87 (sextet, $J=7.6 \mathrm{~Hz}, 2 \mathrm{H}), 1.32(\mathrm{~m}, 6 \mathrm{H}), 0.92(\mathrm{t}, J$ $=6.7 \mathrm{~Hz}) ;{ }^{13} \mathrm{C} \mathrm{NMR}\left(125 \mathrm{MHz}, \mathrm{CDCl}_{3}\right) \delta 163.8,148.9,133.6,130.5,129.3,128.6$, 118.3, 49.7, 31.1, 28.3, 26.3, 22.3, 13.9; MS (FAB) m/z $279\left(\mathrm{M}^{+}\right)$, 260; HRMS calcd for $\mathrm{C}_{18} \mathrm{H}_{18} \mathrm{BF}_{2} \mathrm{NO}_{2}: 280.1684$, found 280.1679 


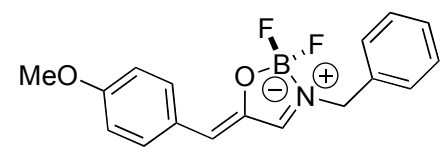

\section{3-Benzyl-5-(4'-methoxybenzylidene)-2,2-difluoro-2,5-dihydro-}

$[1,2,3]$ oxaborazole (9e). Light yellow solid (90 mg, 40\%). $\mathrm{R}_{f}=0.10$ (20\% EtOAc/hex); mp 159-161 ${ }^{\circ} \mathrm{C}$; IR (KBr pellet) 3454, $1578 \mathrm{~cm}^{-1} ;{ }^{1} \mathrm{H}$ NMR (500 MHz, Acetone-d $\left.{ }_{6}\right) \delta$ 7.89 (d, $J=8.8 \mathrm{~Hz}, 2 \mathrm{H}), 7.63(\mathrm{~d}, J=8.4 \mathrm{~Hz}, 2 \mathrm{H}), 7.47$ (d, $J=8.3 \mathrm{~Hz}, 2 \mathrm{H}), 7.00$ (d, $J=$ $8.9 \mathrm{~Hz}, 2 \mathrm{H}), 6.31(\mathrm{~s}, 1 \mathrm{H}), 4.95(\mathrm{~s}, 2 \mathrm{H}), 3.83(\mathrm{~s}, 3 \mathrm{H}) ;{ }^{13} \mathrm{C}$ NMR $\left(125 \mathrm{MHz}\right.$, acetone- $\left.\mathrm{d}_{6}\right) \delta$ $166.0,160.6,133.8,132.0,131.9,127.0,122.0,118.2,114.1,114.0,54.7,51.1$; MS (CIMS) m/z $316\left(\mathrm{M}^{+}+\mathrm{H}\right), 296$; HRMS calcd for $\mathrm{C}_{17} \mathrm{H}_{16} \mathrm{BF}_{2} \mathrm{NO}_{2}$ : 316.1310, found 316.1320.

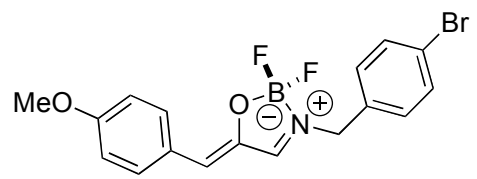

\section{3-(4'-Bromobenzyl)-5-(4'-methoxybenzylidene)-2,2-difluoro-2,5-dihydro-}

[1,2,3]oxaborazole (9f). Light yellow solid (90 mg, 32\%). $\mathrm{R}_{f}=0.07(20 \% \mathrm{EtOAc} / \mathrm{hex})$; mp 196-197 ${ }^{\circ} \mathrm{C}$ (dec); IR (KBr pellet) 3462, $1585 \mathrm{~cm}^{-1} ;{ }^{1} \mathrm{H}$ NMR (400 MHz, DMSO-d 6 $7.83(\mathrm{~d}, J=8.7 \mathrm{~Hz}, 2 \mathrm{H}), 7.62(\mathrm{~d}, J=8.4 \mathrm{~Hz}, 2 \mathrm{H}), 7.37$ (d, $J=8.4 \mathrm{~Hz}) 7.00(\mathrm{~d}, J=8.9 \mathrm{~Hz}$, 2H), $6.38(\mathrm{~s}, 1 \mathrm{H}), 4.87(\mathrm{~s}, 2 \mathrm{H}), 3.83(\mathrm{~s}, 3 \mathrm{H}) ;{ }^{13} \mathrm{C}$ NMR (100 MHz, DMSO-d 6 ) $\delta$ 167.9, $161.0,148.4,134.8,132.8,132.5,132.1,127.4,122.5,119.1,115.2,56.1,51.7$; MS (FAB) $\mathrm{m} / \mathrm{z} 393\left(\mathrm{M}^{+}\right)$, 374; HRMS calcd for $\mathrm{C}_{17} \mathrm{H}_{15} \mathrm{BrBF}_{2} \mathrm{NO}_{2}$ : 393.0347, found 393.0352. 


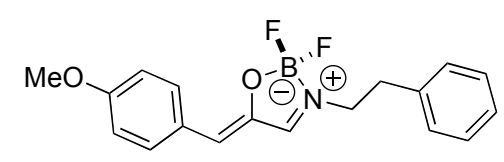

\section{3-Phenylethyl-5-(4'-methoxybenzylidene)-2,2-difluoro-2,5-dihydro-}

$[1,2,3]$ oxaborazole $(9 \mathrm{~g})$. Light yellow crystals $(35 \mathrm{mg}, 30 \%)$ after recrystallization in $\mathrm{CH}_{2} \mathrm{Cl}_{2} / \mathrm{Et}_{2} \mathrm{O} . \mathrm{R}_{f}=0.13$ (20\% EtOAc/hex); mp 153-154 ${ }^{\circ} \mathrm{C}$; IR (KBr pellet) 3480, 1593 $\mathrm{cm}^{-1} ;{ }^{1} \mathrm{H}$ NMR $\left(400 \mathrm{MHz}, \mathrm{CDCl}_{3} \delta 7.84(\mathrm{~d}, J=6.9 \mathrm{~Hz}, 2 \mathrm{H}), 7.29(\mathrm{~m}, 5 \mathrm{H}), 6.90(\mathrm{~d}, J=\right.$ $7.0 \mathrm{~Hz}, 2 \mathrm{H}), 5.88(\mathrm{~s}, 1 \mathrm{H}), 3.96(\mathrm{t}, J=6.8 \mathrm{~Hz}, 2 \mathrm{H}), 3.85(\mathrm{~s}, 3 \mathrm{H}), 3.15(\mathrm{t}, J=6.8 \mathrm{~Hz}) ;{ }^{13} \mathrm{C}$ NMR (100 MHz, $\left.\mathrm{CDCl}_{3}\right) \delta 164.4,161.1,148.0,137.4,133.0,129.5,129.3,127.6,127.0$, 119.4, 114.6, 55.8, 51.5, 35.4; ${ }^{19} \mathrm{~F}$ NMR (471 $\left.\mathrm{MHz}, \mathrm{CDCl}_{3}\right) \delta-150.18,-150.25$ (d); MS (FAB) m/z $329\left(\mathrm{M}^{+}\right), 310$; HRMS calcd for $\mathrm{C}_{18} \mathrm{H}_{18} \mathrm{BF}_{2} \mathrm{NO}_{2}$ : 329.1399, found 329.1409.

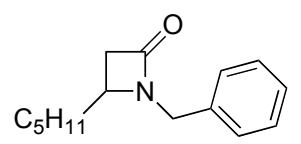

( \pm )-1-Benzyl-4-pentylazetidin-2-one (12). Red oil (11 mg, 12\%). $\mathrm{R}_{f}=0.11(20 \%$ EtOAc/hex); IR (neat) 2924, 2364, $1745 \mathrm{~cm}^{-1} ;{ }^{1} \mathrm{H}$ NMR (500 MHz, $\mathrm{CDCl}_{3} \delta 7.33$ (m, 5H), $4.64(\mathrm{~d}, J=15.3 \mathrm{~Hz}, 1 \mathrm{H}), 4.14(\mathrm{~d}, J=15.2 \mathrm{~Hz}, 1 \mathrm{H}), 3.47(\mathrm{~m}, 1 \mathrm{H}), 3.03(1 \mathrm{H}), 2.60$ $(1 \mathrm{H}), 1.67(\mathrm{~m}, 2 \mathrm{H}) ;{ }^{13} \mathrm{C} \mathrm{NMR}\left(125 \mathrm{MHz}, \mathrm{CDCl}_{3}\right) \delta 167.2,136.1,128.7,127.5,129.3$, 51.4, 44.5, 42.3, 32.7, 31.6, 25.0, 22.4, 13.8; MS (FAB) m/z (M+ ${ }^{+}+$H), 232; HRMS calcd for $\mathrm{C}_{15} \mathrm{H}_{22} \mathrm{NO}$ : 232.1691, found 232.1701. 


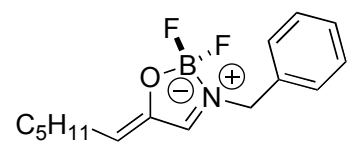

3-Benzyl-5-hexylidene-2,2-difluoro-2,5-dihydro-[1,2,3]oxaborazole (13). Red oil (14 mg, 5\%). $\mathrm{R}_{f}=0.22\left(20 \%\right.$ EtOAc/hex); IR (neat) $2924,1658,1608,1113 \mathrm{~cm}^{-1} ;{ }^{1} \mathrm{H}$ NMR (500 MHz, $\mathrm{CDCl}_{3} \delta 7.47(\mathrm{~m}, 3 \mathrm{H}), 7.41(\mathrm{~s}, 1 \mathrm{H}), 5.29(\mathrm{t}, J=7.7 \mathrm{~Hz}, 1 \mathrm{H}), 4.82(\mathrm{~s}$, 2H), $1.45(\mathrm{p}, J=7.6,6.7 \mathrm{~Hz}, 2 \mathrm{H}), 1.32(\mathrm{~m}, 6 \mathrm{H}), 0.89(\mathrm{t}, J=6.8 \mathrm{~Hz}) ;{ }^{13} \mathrm{C} \mathrm{NMR}(125$ $\left.\mathrm{MHz}, \mathrm{CDCl}_{3}\right) \delta 162.7,150.0,132.1,129.7,129.5,129.3,124.0,51.8,31.4,28.2,26.1$, 22.3, 13.9; MS (FAB) m/z $\left(\mathrm{M}^{+}\right), 279,260$; HRMS calcd for $\mathrm{C}_{15} \mathrm{H}_{21} \mathrm{BF}_{2} \mathrm{NO}$ : 280.1684, found 280.1660 .

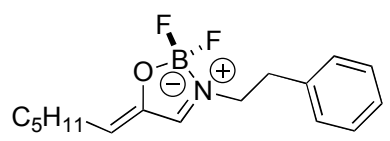

\section{3-Phenylethyl-5-hexylidene-2,2-difluoro-2,5-dihydro-[1,2,3]oxaborazole}

(14a). Isolated as a ca. 4:1, Z:E mixture (red oil, $6 \mathrm{mg}, 6 \%) . \mathrm{R}_{f}=0.18(20 \% \mathrm{EtOAc} / \mathrm{hex})$; IR (neat) 2924, $1083 \mathrm{~cm}^{-1}$; MS (FAB) m/z $294\left(\mathrm{M}^{+}\right), 274 ;$ HRMS calcd for $\mathrm{C}_{16} \mathrm{H}_{23} \mathrm{BF}_{2} \mathrm{NO}: 294.1841$, found 294.1856. $Z$-isomer: ${ }^{1} \mathrm{H} \mathrm{NMR}\left(400 \mathrm{MHz}, \mathrm{CDCl}_{3}\right) \delta 7.25$ $(\mathrm{m}, 5 \mathrm{H}), 5.22(\mathrm{t}, J=7.7 \mathrm{~Hz}, 1 \mathrm{H}), 3.94(\mathrm{t}, J=6.7,2 \mathrm{H}), 3.14(\mathrm{t}, J=6.8 \mathrm{~Hz}, 2 \mathrm{H}), 2.37(\mathrm{q}, J$ $=7.4 \mathrm{~Hz}, 2 \mathrm{H}), 1.38(\mathrm{~m}, 6 \mathrm{H}) 0.90(\mathrm{t}, J=6.7 \mathrm{~Hz}, 3 \mathrm{H}){ }^{13} \mathrm{C} \mathrm{NMR}\left(100 \mathrm{MHz}, \mathrm{CDCl}_{3}\right) \delta$ $164.0,149.8,137.3,129.5,129.3,127.6,124.0,51.6,35.2,31.9,28.7,26.6,22.8,14.4$. E-isomer: ${ }^{1} \mathrm{H}$ NMR $\left(400 \mathrm{MHz}, \mathrm{CDCl}_{3}\right) \delta 5.32(\mathrm{~m}, 1 \mathrm{H}), 3.84(\mathrm{t}, J=8.0,2 \mathrm{H}), 2.19(\mathrm{~d}, J=$ $0.6 \mathrm{~Hz})$. 


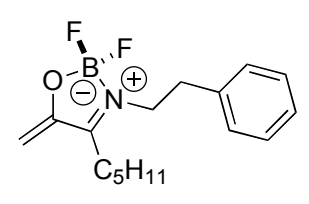

\section{3-Phenylethyl-4-hexylidene-5-methylene-2,2-difluoro-2,5-dihydro-}

$[1,2,3]$ oxaborazole (14b). Yellow oil $7.0 \mathrm{mg}(8 \%) . \mathrm{R}_{f}=0.20(20 \%$ EtOAc/hex); IR (neat) $2924,1774,1644,1098 \mathrm{~cm}^{-1} ;{ }^{1} \mathrm{H}$ NMR $\left(500 \mathrm{MHz}, \mathrm{CDCl}_{3} \delta 7.28(\mathrm{~m}, 5 \mathrm{H}), 5.1\right.$ (complex, 1H), $4.84(\mathrm{~d}, J=2.3 \mathrm{~Hz}, 1 \mathrm{H}), 3.88(\mathrm{t}, J=7.0 \mathrm{~Hz}, 2 \mathrm{H}), 3.18(\mathrm{t}, J=7.0 \mathrm{~Hz}, 2 \mathrm{H})$, $2.11(\mathrm{t}, J=7.5 \mathrm{~Hz}, 2 \mathrm{H}), 1.28(\mathrm{~m}, 8 \mathrm{H}), 0.90(\mathrm{t}, J=6.8 \mathrm{~Hz}, 2 \mathrm{H}) ;{ }^{13} \mathrm{C} \mathrm{NMR}(125 \mathrm{MHz}$, $\left.\mathrm{CDCl}_{3}\right) \delta 177.0,154.6,137.4,129.0,128.8,127.1,97.0,48.0,34.4,31.7,27.7,25.1$, 21.9, 13.6; MS (FAB) m/z $295\left(\mathrm{M}^{+}+\mathrm{H}\right), 274$; HRMS calcd for $\mathrm{C}_{16} \mathrm{H}_{23} \mathrm{BF}_{2} \mathrm{NO}$ : 294.1841, found 294.1856.

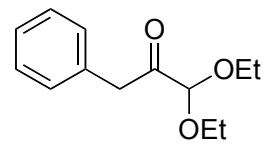

Acidic ethanolysis of a 1,2,3[oxaborazole]. To a solution of $20 \mathrm{mg}$ of $9 \mathrm{a}$ in 10 $\mathrm{mL}$ anhydrous $50 \% \mathrm{CH}_{2} \mathrm{Cl}_{2} / \mathrm{EtOH}$ was added 2-3 drops of concentrated $\mathrm{HCl}$ at room temperature. The reaction mixture was stirred for 12 hours, then quenched with $10 \mathrm{~mL}$ saturated $\mathrm{NaHCO}_{3}$ and extracted with $\mathrm{CH}_{2} \mathrm{Cl}_{2}(3 \times 10 \mathrm{~mL})$. The organic extracts were then dried with $\mathrm{MgSO}_{4}$, filtered and chromatographed (10\%EtOAc/hex) to provide a clear oil (15.6 mg, ca. 100\%). The spectra recorded for this compound match those reported previously. ${ }^{4}$ 


\section{Molecular modeling data}

Table 1S. B3LYP/6-31+G(d) cartesian coordinates and energy for methyl azide.

\begin{tabular}{|l|c|c|c|}
\hline Atom & $\mathrm{X}$ & $\mathrm{Y}$ & $\mathrm{Z}$ \\
\hline $\mathrm{N}$ & 0.68184 & -0.30538 & 0.00000 \\
\hline $\mathrm{C}$ & -0.07373 & -1.57279 & 0.00000 \\
\hline $\mathrm{H}$ & -0.70139 & -1.66713 & 0.89526 \\
\hline $\mathrm{H}$ & -0.70139 & -1.66713 & -0.89526 \\
\hline $\mathrm{H}$ & 0.66682 & -2.37291 & 0.00000 \\
\hline $\mathrm{N}$ & 0.00000 & 0.72358 & 0.00000 \\
\hline $\mathrm{N}$ & -0.51350 & 1.74521 & 0.00000 \\
\hline Energy (HF) & -204.1016588 \\
\hline
\end{tabular}

Table 2S. B3LYP/6-31+G(d) cartesian coordinates and energy for the methyl substituted oxyallyl cation (a in Figure 3).

\begin{tabular}{|l|c|c|c|}
\hline Atom & $\mathrm{X}$ & $\mathrm{Y}$ & $\mathrm{Z}$ \\
\hline $\mathrm{C}$ & 0.96364 & 0.50201 & -0.00182 \\
\hline $\mathrm{O}$ & 0.13622 & -0.50204 & -0.00158 \\
\hline $\mathrm{C}$ & 2.35226 & 0.23190 & -0.00170 \\
\hline $\mathrm{H}$ & 3.03805 & 1.07513 & -0.00420 \\
\hline $\mathrm{C}$ & 0.64157 & 1.86220 & 0.00136 \\
\hline $\mathrm{H}$ & -0.38775 & 2.20228 & 0.00690 \\
\hline $\mathrm{H}$ & 1.43409 & 2.60294 & -0.00130 \\
\hline $\mathrm{C}$ & 2.86827 & -1.14068 & 0.00169 \\
\hline $\mathrm{H}$ & 3.95718 & -1.19909 & 0.00749 \\
\hline $\mathrm{H}$ & 2.44387 & -1.68370 & 0.86246 \\
\hline $\mathrm{H}$ & 2.45446 & -1.68268 & -0.86512 \\
\hline $\mathrm{B}$ & -1.39425 & -0.26088 & -0.00007 \\
\hline $\mathrm{F}$ & -1.68128 & 0.48787 & 1.15070 \\
\hline $\mathrm{F}$ & -1.97066 & -1.50506 & -0.00188 \\
\hline $\mathrm{F}$ & -1.68283 & 0.49199 & -1.14775 \\
\hline Energy (HF) & -555.7900017 \\
\hline
\end{tabular}


Table 3S. B3LYP $/ 6-31+\mathrm{G}(\mathrm{d})$ cartesian coordinates and energy for the dimethyl substituted oxyallyl cation (b in Figure 3).

\begin{tabular}{|l|c|c|c|}
\hline Atom & $\mathrm{X}$ & $\mathrm{Y}$ & \multicolumn{1}{c|}{$\mathrm{Z}$} \\
\hline $\mathrm{C}$ & -0.67578 & 0.57930 & -0.38660 \\
\hline $\mathrm{O}$ & 0.40460 & -0.02688 & -0.84066 \\
\hline $\mathrm{C}$ & -1.80930 & -0.25101 & -0.08342 \\
\hline $\mathrm{C}$ & -0.73265 & 1.93390 & -0.13291 \\
\hline $\mathrm{H}$ & 0.16681 & 2.52848 & -0.25129 \\
\hline $\mathrm{H}$ & -1.62799 & 2.42571 & 0.22574 \\
\hline $\mathrm{C}$ & -1.58282 & -1.70765 & -0.11952 \\
\hline $\mathrm{H}$ & -2.46744 & -2.29112 & 0.14235 \\
\hline $\mathrm{H}$ & -1.20677 & -1.98969 & -1.11513 \\
\hline $\mathrm{H}$ & -0.73396 & -1.94372 & 0.54351 \\
\hline $\mathrm{C}$ & -3.17467 & 0.23215 & 0.24512 \\
\hline $\mathrm{H}$ & -3.87908 & -0.19985 & -0.48178 \\
\hline $\mathrm{H}$ & -3.47538 & -0.17564 & 1.22093 \\
\hline $\mathrm{H}$ & -3.30046 & 1.31398 & 0.24981 \\
\hline $\mathrm{B}$ & 1.64800 & -0.11037 & 0.06269 \\
\hline F & 2.60972 & -0.77470 & -0.66016 \\
\hline F & 2.02229 & 1.19393 & 0.41624 \\
\hline F & 1.24563 & -0.82160 & 1.21522 \\
\hline Energy & -595.1109659 \\
\hline
\end{tabular}

Table 4S. B3LYP/6-31+G(d) cartesian coordinates and energy for the phenyl substituted oxyallyl cation (c in Figure 3).

\begin{tabular}{|l|c|c|c|}
\hline Atom & $\mathrm{X}$ & $\mathrm{Y}$ & $\mathrm{Z}$ \\
\hline $\mathrm{C}$ & -0.81296 & 1.07935 & -0.00012 \\
\hline $\mathrm{O}$ & -1.17699 & -0.17480 & -0.00021 \\
\hline $\mathrm{C}$ & 0.57755 & 1.41315 & 0.00000 \\
\hline $\mathrm{C}$ & -1.66623 & 2.17707 & -0.00023 \\
\hline $\mathrm{H}$ & -2.74255 & 2.05363 & -0.00047 \\
\hline $\mathrm{H}$ & -1.26012 & 3.18233 & -0.00005 \\
\hline $\mathrm{H}$ & 0.79969 & 2.47714 & 0.00011 \\
\hline $\mathrm{C}$ & 1.69854 & 0.54507 & -0.00004 \\
\hline $\mathrm{C}$ & 1.59287 & -0.87810 & -0.00021 \\
\hline $\mathrm{C}$ & 2.74473 & -1.65402 & -0.00019 \\
\hline $\mathrm{C}$ & 4.00838 & -1.04881 & 0.00002 \\
\hline $\mathrm{C}$ & 4.13476 & 0.35047 & 0.00018 \\
\hline $\mathrm{C}$ & 2.99656 & 1.13790 & 0.00014 \\
\hline $\mathrm{H}$ & 0.60915 & -1.33029 & -0.00033 \\
\hline $\mathrm{H}$ & 2.66167 & -2.73675 & -0.00032 \\
\hline $\mathrm{H}$ & 4.90187 & -1.66783 & 0.00005 \\
\hline $\mathrm{H}$ & 5.11902 & 0.80922 & 0.00034 \\
\hline
\end{tabular}


Grecian et al.

\begin{tabular}{|l|c|c|c|}
\hline $\mathrm{H}$ & 3.08255 & 2.22181 & 0.00024 \\
\hline $\mathrm{B}$ & -2.65769 & -0.61122 & 0.00005 \\
\hline $\mathrm{F}$ & -3.25058 & -0.07680 & -1.15018 \\
\hline $\mathrm{F}$ & -3.25021 & -0.07646 & 1.15041 \\
\hline $\mathrm{F}$ & -2.62278 & -1.98978 & 0.00027 \\
\hline Energy & -747.5532049 \\
\hline
\end{tabular}

Table 5S. Optimized cartesian coordinates and energy for methyl azide at the B3LYP/6$31+\mathrm{G}(\mathrm{d})$, IEFPCM (solvent $=$ dichloromethane) level of theory.

\begin{tabular}{|l|c|c|c|}
\hline Atom & $\mathrm{X}$ & $\mathrm{Y}$ & $\mathrm{Z}$ \\
\hline $\mathrm{N}$ & 0.67892 & -0.30255 & 0.00000 \\
\hline $\mathrm{C}$ & -0.07207 & -1.57839 & 0.00000 \\
\hline $\mathrm{H}$ & -0.69746 & -1.66878 & 0.89626 \\
\hline $\mathrm{H}$ & -0.69746 & -1.66878 & -0.89626 \\
\hline $\mathrm{H}$ & 0.67362 & -2.37379 & 0.00000 \\
\hline $\mathrm{N}$ & 0.00000 & 0.72505 & 0.00000 \\
\hline $\mathrm{N}$ & -0.51410 & 1.74631 & 0.00000 \\
\hline \multicolumn{2}{|l|}{ Energy } & -204.105851 \\
\hline
\end{tabular}

Table 6S. Optimized cartesian coordinates and energy for the methyl substituted oxyallyl cation at the B3LYP/6-31+G(d), IEFPCM (solvent = dichloromethane) level of theory.

\begin{tabular}{|l|c|c|c|}
\hline Atom & X & Y & Z \\
\hline C & -0.97935 & 0.51872 & -0.00018 \\
\hline O & -0.10239 & -0.47155 & -0.00069 \\
\hline C & -2.35737 & 0.20073 & -0.00002 \\
\hline H & -3.06366 & 1.03392 & 0.00047 \\
\hline C & -0.70208 & 1.87760 & -0.00005 \\
\hline H & 0.31255 & 2.26323 & -0.00041 \\
\hline H & -1.51967 & 2.59292 & 0.00016 \\
\hline C & -2.89664 & -1.15760 & 0.00006 \\
\hline H & -2.12621 & -1.92877 & 0.00164 \\
\hline H & -3.56694 & -1.27363 & 0.86936 \\
\hline H & -3.56369 & -1.27455 & -0.87170 \\
\hline B & 1.37378 & -0.24966 & 0.00003 \\
\hline F & 1.74535 & 0.47804 & -1.15052 \\
\hline F & 1.96513 & -1.51451 & 0.00006 \\
\hline F & 1.74401 & 0.47767 & 1.15123 \\
\hline Energy & -555.8204906 \\
\hline
\end{tabular}


Table 7S. Optimized cartesian coordinates and energy for the dimethyl substituted oxyallyl cation at the B3LYP/6-31+G(d), IEFPCM (solvent = dichloromethane) level of theory.

\begin{tabular}{|l|c|c|c|}
\hline Atom & X & Y & Z \\
\hline C & -0.69556 & 0.58156 & -0.35394 \\
\hline O & 0.40126 & -0.02154 & -0.80727 \\
\hline C & -1.82975 & -0.25221 & -0.07335 \\
\hline C & -0.75773 & 1.92486 & -0.07680 \\
\hline H & 0.14117 & 2.52610 & -0.15825 \\
\hline H & -1.66423 & 2.41419 & 0.25841 \\
\hline C & -1.59400 & -1.70919 & -0.06620 \\
\hline H & -2.45032 & -2.28013 & 0.29636 \\
\hline H & -1.34831 & -2.02167 & -1.09486 \\
\hline H & -0.68174 & -1.92299 & 0.50655 \\
\hline C & -3.20422 & 0.23074 & 0.18539 \\
\hline H & -3.87552 & -0.25684 & -0.53885 \\
\hline H & -3.53342 & -0.13270 & 1.17037 \\
\hline H & -3.34441 & 1.30886 & 0.12303 \\
\hline B & 1.64056 & -0.10235 & 0.04615 \\
\hline F & 2.64448 & -0.59749 & -0.77011 \\
\hline F & 1.96008 & 1.16919 & 0.53852 \\
\hline F & 1.37670 & -0.97229 & 1.11760 \\
\hline Energy (HF) & -595.0950366 \\
\hline
\end{tabular}

Table 8S. Optimized cartesian coordinates and energy for the phenyl substituted oxyallyl cation at the B3LYP/6-31+G(d), IEFPCM (solvent = dichloromethane) level of theory.

\begin{tabular}{|l|c|c|c|}
\hline Atom & $\mathrm{X}$ & $\mathrm{Y}$ & $\mathrm{Z}$ \\
\hline $\mathrm{C}$ & -0.81082 & 1.11482 & 0.00430 \\
\hline $\mathrm{O}$ & -1.20563 & -0.15405 & 0.00453 \\
\hline $\mathrm{C}$ & 0.59227 & 1.40598 & 0.00319 \\
\hline $\mathrm{C}$ & -1.63566 & 2.21729 & 0.00209 \\
\hline $\mathrm{H}$ & -2.71632 & 2.12870 & 0.00088 \\
\hline $\mathrm{H}$ & -1.20745 & 3.21445 & 0.00209 \\
\hline H & 0.83202 & 2.46987 & 0.00096 \\
\hline $\mathrm{C}$ & 1.69951 & 0.53165 & 0.00281 \\
\hline $\mathrm{C}$ & 1.60131 & -0.89462 & 0.00655 \\
\hline $\mathrm{C}$ & 2.75328 & -1.66475 & 0.00406 \\
\hline $\mathrm{C}$ & 4.01634 & -1.04938 & -0.00196 \\
\hline $\mathrm{C}$ & 4.13860 & 0.35031 & -0.00565 \\
\hline $\mathrm{C}$ & 2.99742 & 1.13336 & -0.00324 \\
\hline $\mathrm{H}$ & 0.62377 & -1.35795 & 0.01156 \\
\hline $\mathrm{H}$ & 2.67922 & -2.75091 & 0.00695 \\
\hline $\mathrm{H}$ & 4.91436 & -1.66708 & -0.00384 \\
\hline
\end{tabular}




\begin{tabular}{|l|l|l|l|}
\hline $\mathrm{H}$ & 5.12370 & 0.81333 & -0.01069 \\
\hline $\mathrm{H}$ & 3.07570 & 2.22050 & -0.00685 \\
\hline $\mathrm{B}$ & -2.62808 & -0.58704 & -0.00169 \\
\hline $\mathrm{F}$ & -3.28593 & -0.09070 & -1.14800 \\
\hline $\mathrm{F}$ & -3.28672 & -0.11788 & 1.15546 \\
\hline $\mathrm{F}$ & -2.61103 & -1.98822 & -0.01875 \\
\hline Energy (HF) & -747.5868788 \\
\hline
\end{tabular}

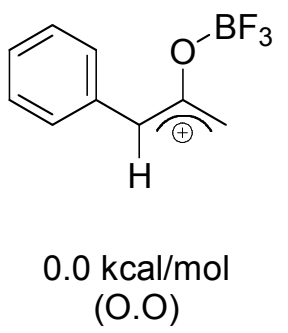

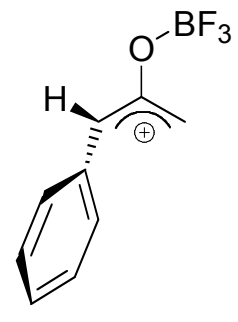

$9.1 \mathrm{kcal} / \mathrm{mol}$ (6.0)

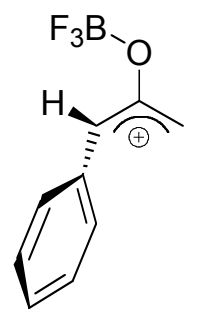

$5.9 \mathrm{kcal} / \mathrm{mol}$

(6.1)

Figure S1. B3LYP/6-31+G(d) relative energies of the phenyl substituted oxyallyl cation conformers. Numbers in parentheses are from optimized structures with the IEFPCM solvent model (solvent $=$ dichloromethane). 


\section{Depictions of X-ray structures}
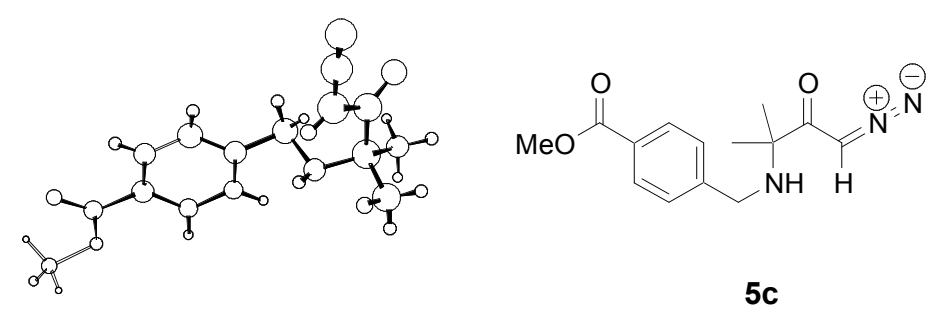

Figure S2. Ball-and-stick depiction of the X-ray crystallographic analysis of $\alpha$-amino- $\alpha^{\prime}-$ diazomethyl ketone 5c. The details of this structure have been previously published. ${ }^{4}$
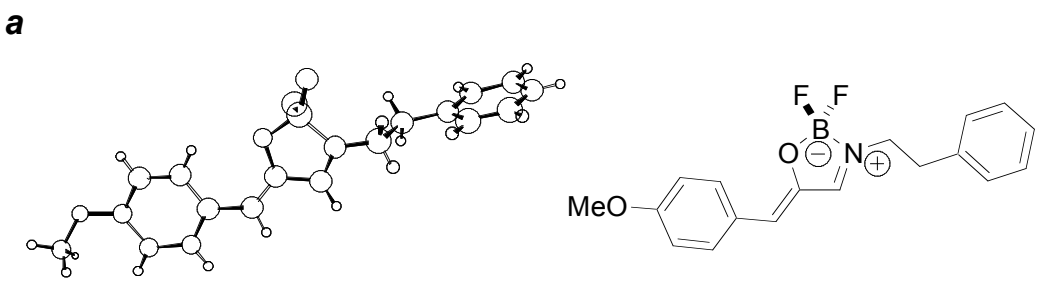

$9 \mathrm{~g}$

Figure S3. Ball-and-stick depiction of X-ray determination of 9g. Details of this structure are provided in the CIF file accompanying this paper.

\section{References for Supporting Information}

(1) Angeles, E.; Santillan, A.; Martinez, I.; Ramirez, A.; Moreno, E.; Salmon, M.; Martinez, R. Synth. Commun. 1994, 24, 2441-2447.

(2) Ainsworth, C.; Chen, F.; Kuo, Y.-N. J. Organomet. Chem. 1972, 46, 59-71.

(3) Rousseau, G.; Slougui, N. Tetrahedron Lett. 1983, 24, 1251-1254.

(4) Desai, P.; Aubé, J. Org. Lett. 2000, 2, 1657-1659.

(5) Adamczyk, M.; Johnson, D. D.; Mattingly, P. G.; Pan, Y.; Reddy, R. E. Synth. Commun. 2002, 32, 3199-3205. 


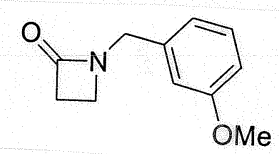

$2 c$
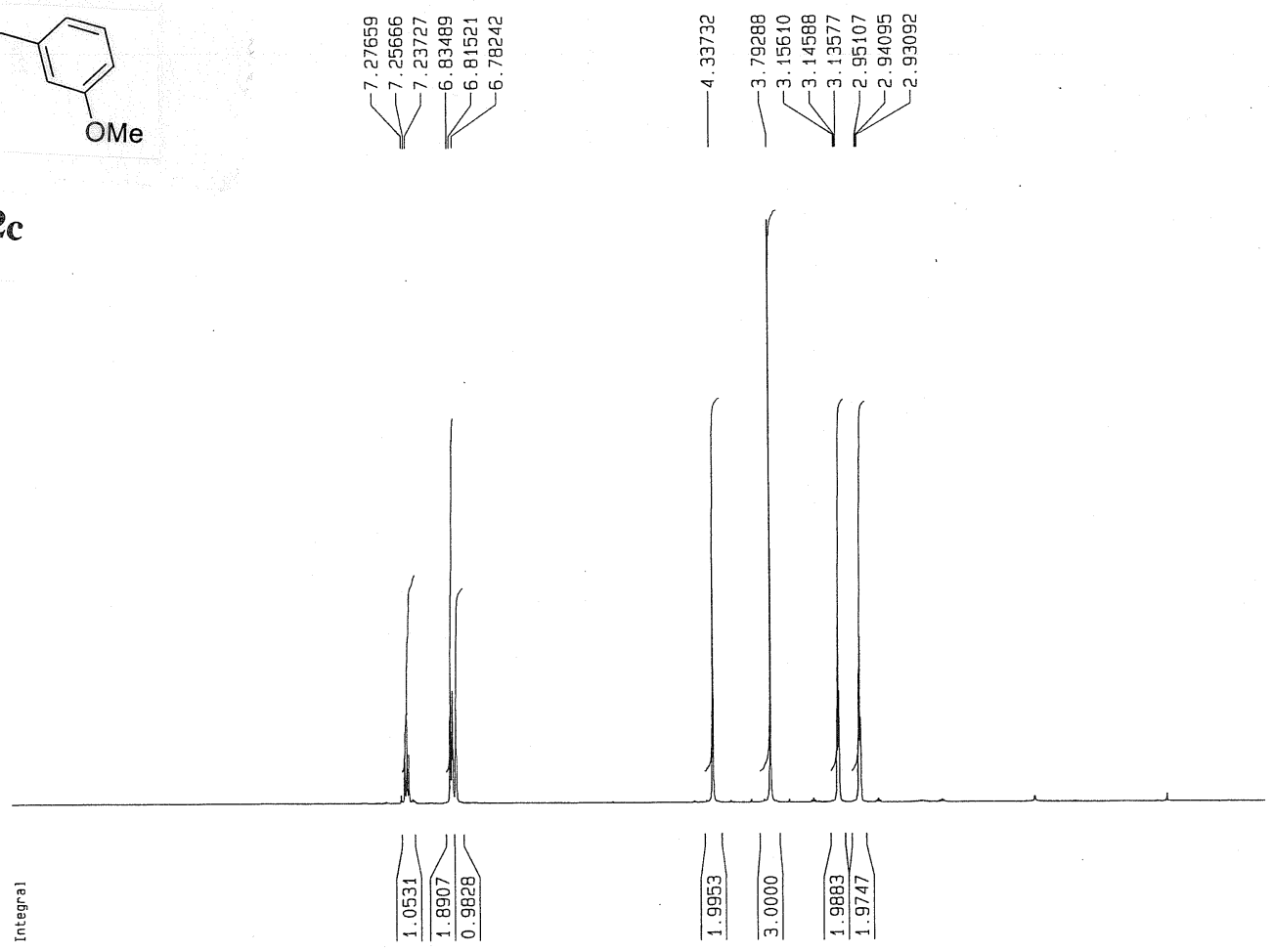

$\begin{array}{lll} & \\ \mathrm{ppm} & 10 & \end{array}$

言
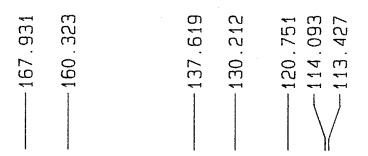

高品啹

iñ

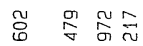

V
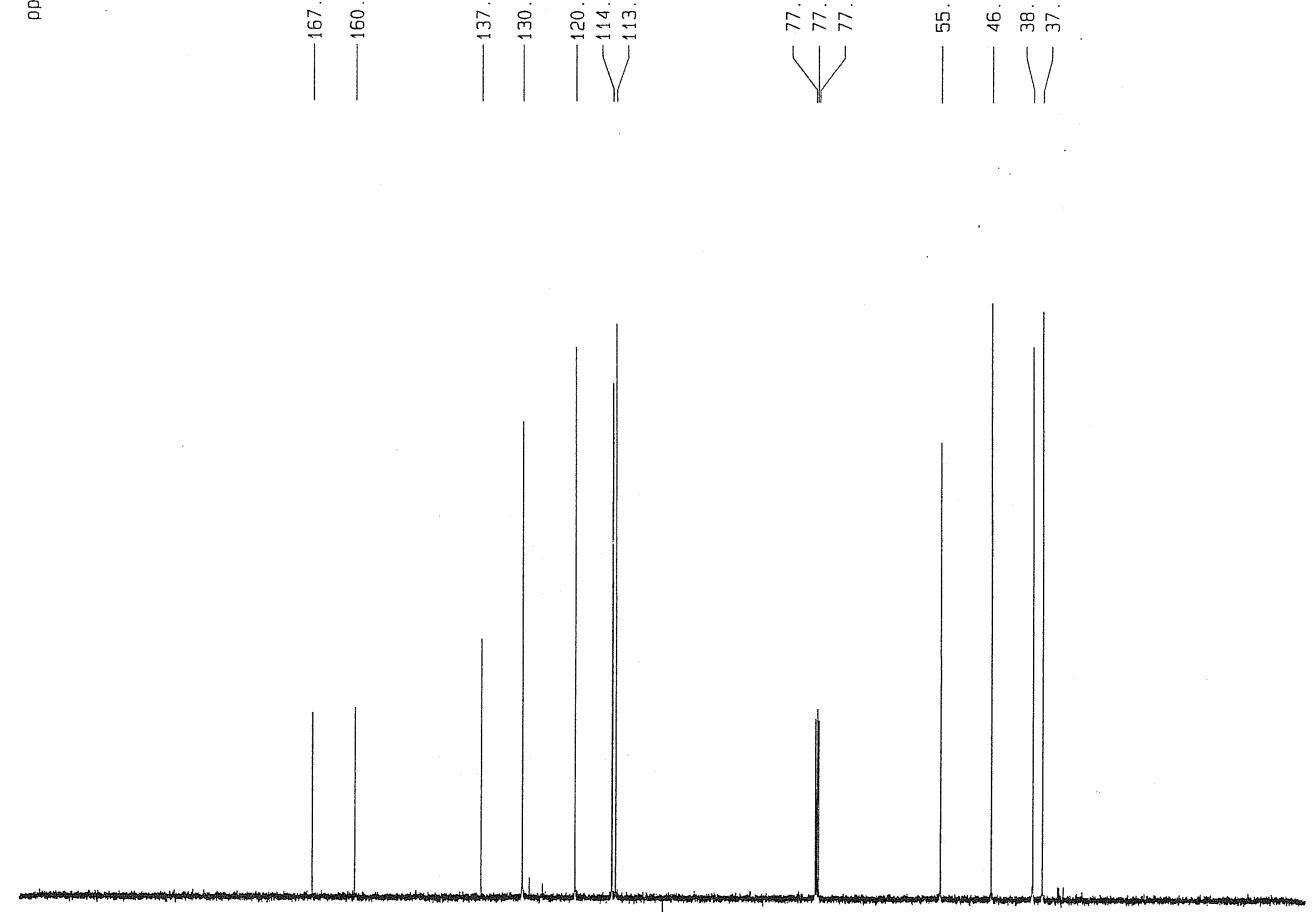

ppm
Current Data Parameters

NAME

PROCNO 1

F2 - Acquisition Parameters

Date_ $\quad 990616$

INSTRUM 17.21

PROBHD $5 \mathrm{~mm}$ Multinu

PULPROG $\quad 2930$

TD 32768

$\begin{array}{lr}\text { SOLVENT } & \text { CDC13 } \\ \text { NS } & 8 \\ \text { DS } & 2\end{array}$

$\begin{array}{ll}\text { SWH } & 4789.272 \mathrm{~Hz} \\ \text { FIDRES } & 0.146157 \mathrm{~Hz}\end{array}$

3. $4210291 \mathrm{sec}$

104. 400 usec

4.50 use

$1.300 .0 \mathrm{~K}$

7.70 usec

400. $1320007 \mathrm{MHz}$

$\begin{array}{lr}\text { NUC1 } & 1 \mathrm{H} \\ \text { PL.1 } & -6.00 \mathrm{~dB}\end{array}$

F2 - Processing parameters

SI 16384

SF $\quad 400.1299840$

$\begin{array}{lc}\text { SSB } & 0 \\ L B & 0.30 \mathrm{~Hz}\end{array}$

$\begin{array}{lr}\text { GB } & 0 \\ \text { PC } & 1.00\end{array}$

10 NMF plot parameters

$\begin{array}{lc}\text { CX } & 20.00 \mathrm{~cm} \\ \text { FIP } & 11.025 \mathrm{ppm}\end{array}$

F1 $\quad 4411.31 \mathrm{~Hz}$

$-0.945 \mathrm{ppm}$
$-377.96 \mathrm{~Hz}$
$0.5946 \mathrm{pm}$

$0.59846 \mathrm{ppm} / \mathrm{c}$

$239.46358 \mathrm{~Hz} / \mathrm{cm}$
Current Data Parameters NAME pnd-ij-4ia

EXPND

F2 - ACqujsition Parameters

Date $\quad 990616$

17.26
INSTRUM

PROBHD $5 \mathrm{~mm}$ Multinu

$\begin{array}{ll}\text { PULPAOG } & \text { zgpg30 } \\ & 65535\end{array}$

SOLVENT

NS
DS

23148.148 Hz

$0.353213 \mathrm{~Hz}$

1. $4156276 \mathrm{sec}$

2048

4.50 usec

$300.0 \mathrm{~K}$

$0.0300000 \mathrm{sec}$

$0.0000200 \mathrm{sec}$

$18.00 \mathrm{~dB}$

$.05000000 \mathrm{sec}$

wa1tz16

100.00 usec

1.

$1 \mathrm{H}$
$-6.00 \mathrm{~dB}$

$18.00 \mathrm{~dB}$

6.90 usec

$\begin{array}{lr}\text { DEF } & 100.6232993 \mathrm{MHZ} \\ \text { NUC1 } & 13 \mathrm{C} \\ \text { PL1 } & -6.00 \mathrm{~dB}\end{array}$

F2 - Processing parameters

$\begin{array}{lc}\text { F2 } & \text { Processing parameters } \\ \text { SI } & 32768 \\ \text { SF } & 100.6127290 \mathrm{MHz}\end{array}$

WDW $100.612729 \mathrm{EM}$

$\begin{array}{lc}\mathrm{LB} & 0 \\ \mathrm{CB} & 1.00 \mathrm{~Hz}\end{array}$

PC $\quad 1.40$

10 NMA plot parameters

$\begin{array}{ll}\text { CX } & 20.00 \mathrm{~cm} \\ \text { FIP } & \text { Fi }\end{array}$

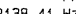

$-10.036 \mathrm{pp}$

$-1009.74 \mathrm{~Hz}$

$11.50359 \mathrm{ppm} / \mathrm{cm}$ $1157.40747 \mathrm{~Hz} / \mathrm{cm}$ 

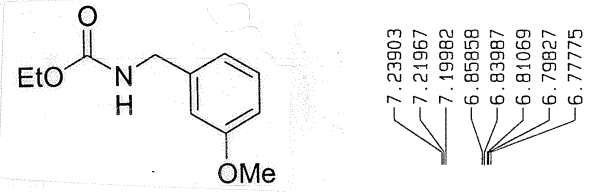

$3 c$

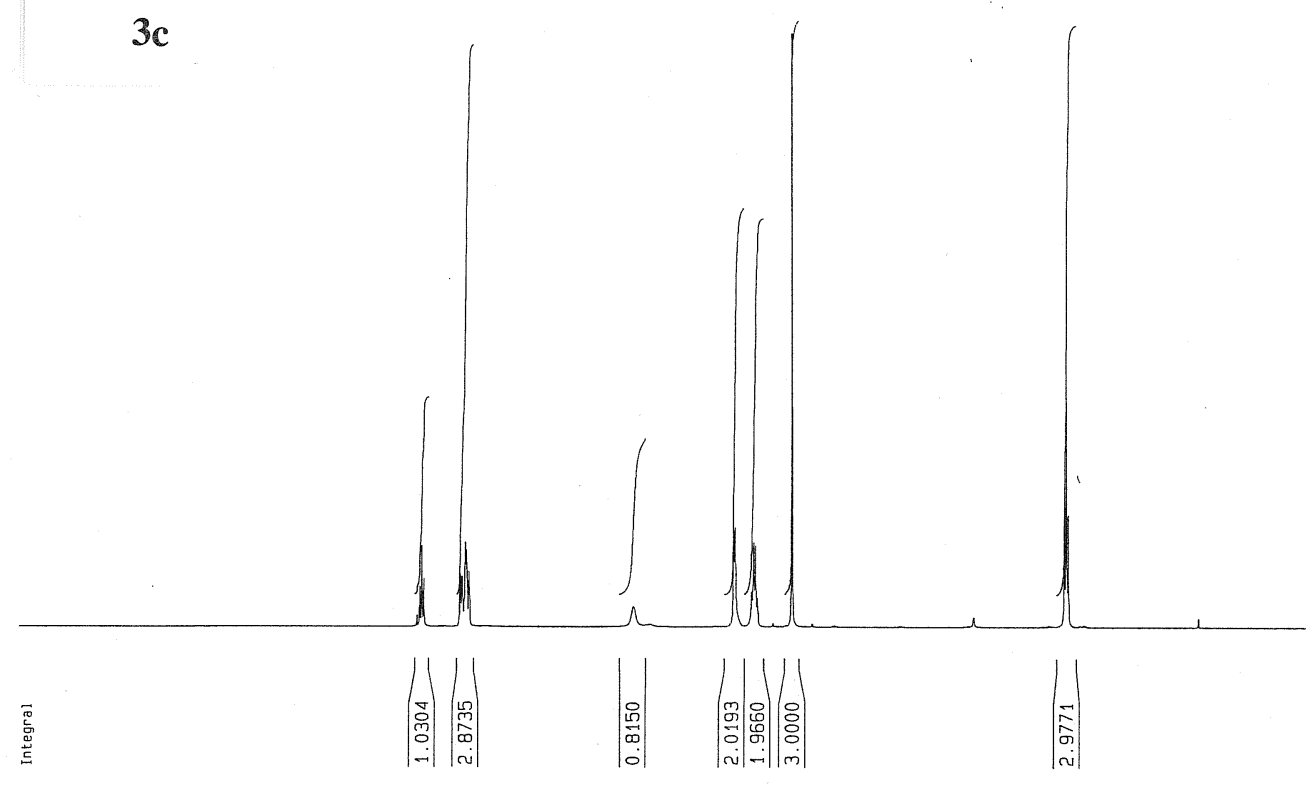

ppm

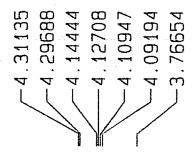

员罣余

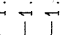

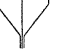

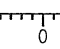

言

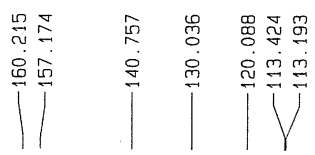

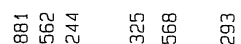

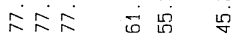

V

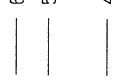

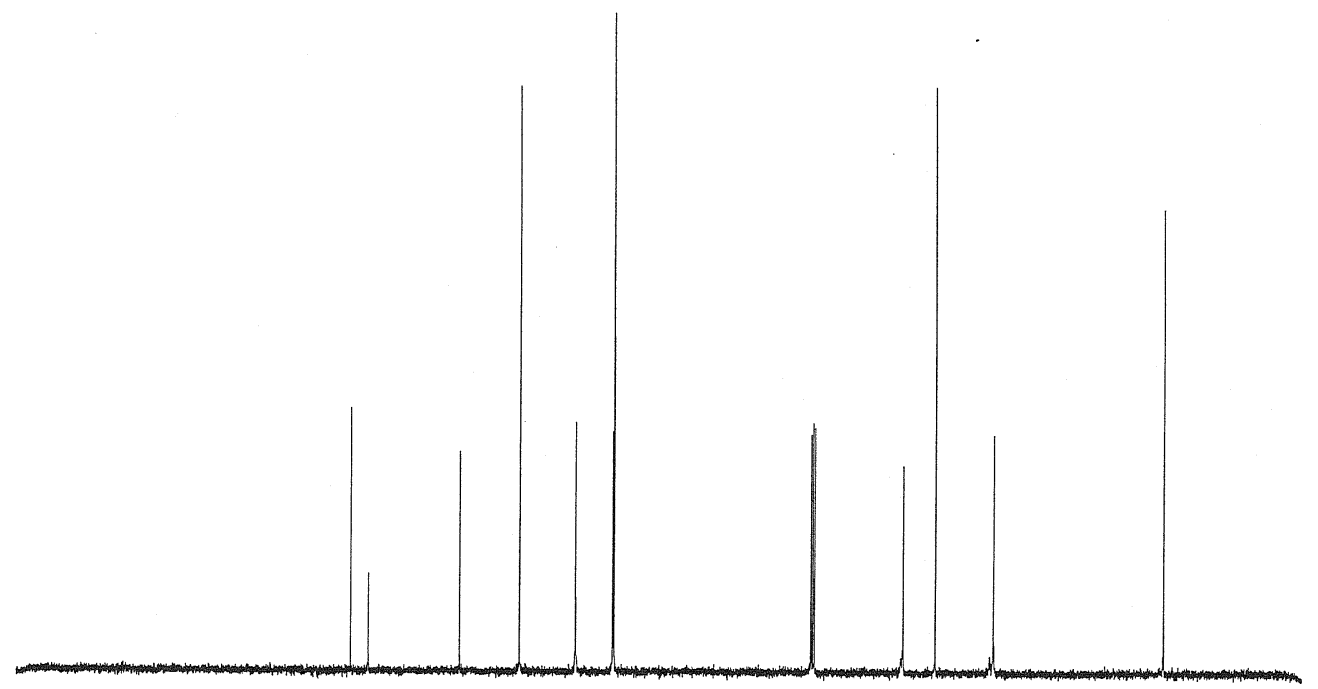

ppm

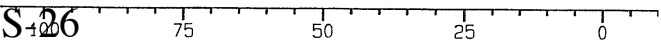

Current Data Parameters

NAME
EXPNO

PROCNO

Fa - Acquisition Parameters

Date__ 990617

$\begin{array}{lr}\text { Time } & 17.00 \\ \text { INSTRUM } & \text { d } \times 400\end{array}$

PROBHD $5 \mathrm{~mm}$ Multinu

$\begin{array}{lr}\text { PULPROG } & 2930 \\ & 32768\end{array}$

SOLVENT CDC13

DS

SWH $\quad 4789.272 \mathrm{~Hz}$

IDRES $\quad 0.146157 \mathrm{~Hz}$

$\begin{array}{lc}A G & 3.4210291 \mathrm{sec} \\ \mathrm{AG} & 32\end{array}$

$\begin{array}{lr}\mathrm{DW} & 104.400 \text { usec } \\ \mathrm{DE} & 4.50 \text { usec }\end{array}$

$4.50 \mathrm{usec}$
$300.0 \mathrm{~K}$

$00000000 \mathrm{sec}$

7.70 usec

400.1320007 MHz

$-6.00 \mathrm{~dB}$

F2 - Processing parameters

$\begin{array}{cc}\text { SI } & 16384 \\ \text { SF } & 400.1300074\end{array}$

WOW $\quad 400.1300074$ EM

$\begin{array}{cc} & 0 \\ \mathrm{~B} & 0.30 \mathrm{~Hz}\end{array}$

1.00

10 NMR plot parameters

CX $\quad 20.00 \mathrm{~cm}$

$\begin{array}{lr}F_{1} & 10.966 \mathrm{pp} \\ \mathrm{F} & 4387.93 \mathrm{~Hz}\end{array}$

$\begin{array}{ll}F 2 P & 4387.93 \mathrm{~Hz} \\ F & -1.003 \mathrm{ppm}\end{array}$

РPMCM $\quad 0.59846 \mathrm{pom} / \mathrm{cm}$

HZCM $239.4635 \mathrm{~B} \mathrm{~Hz} / \mathrm{cm}$

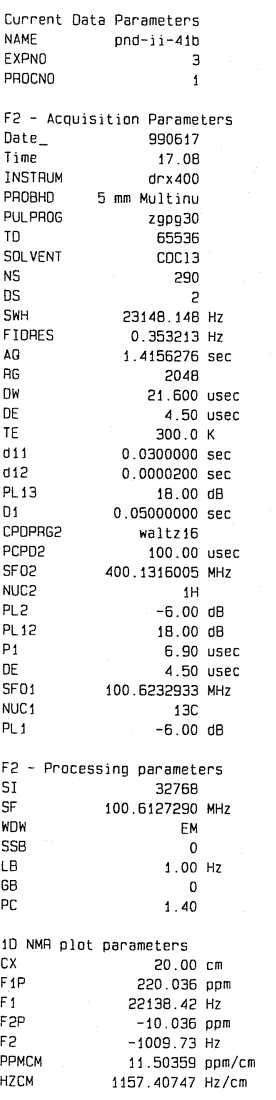



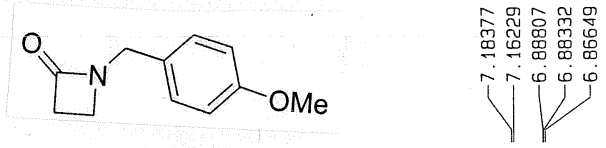

$2 d$
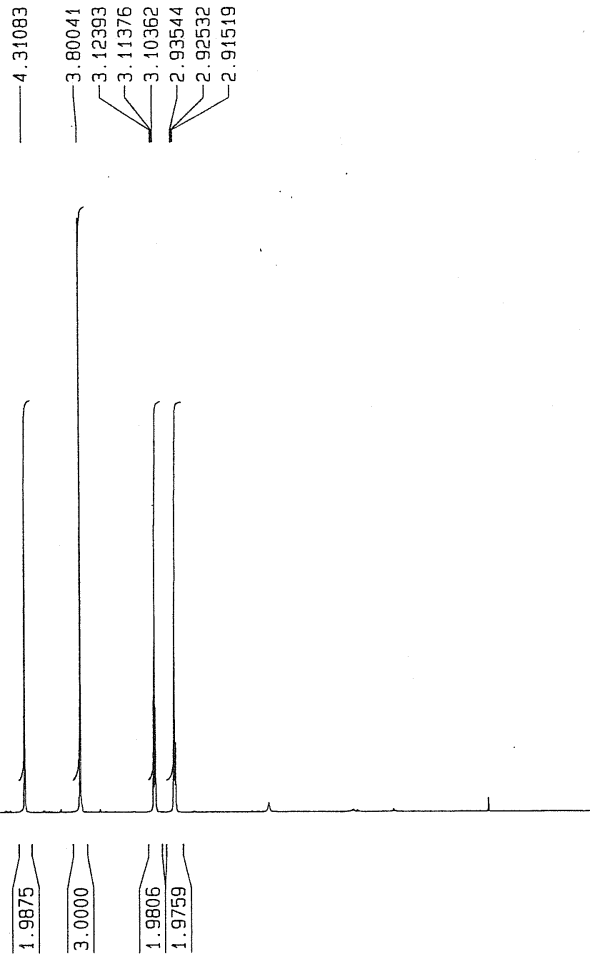

ppm

틈
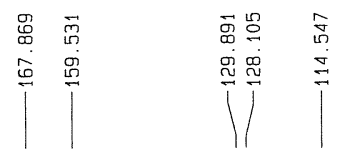

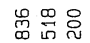

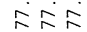

V

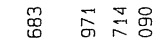

员皆离向

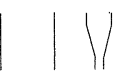

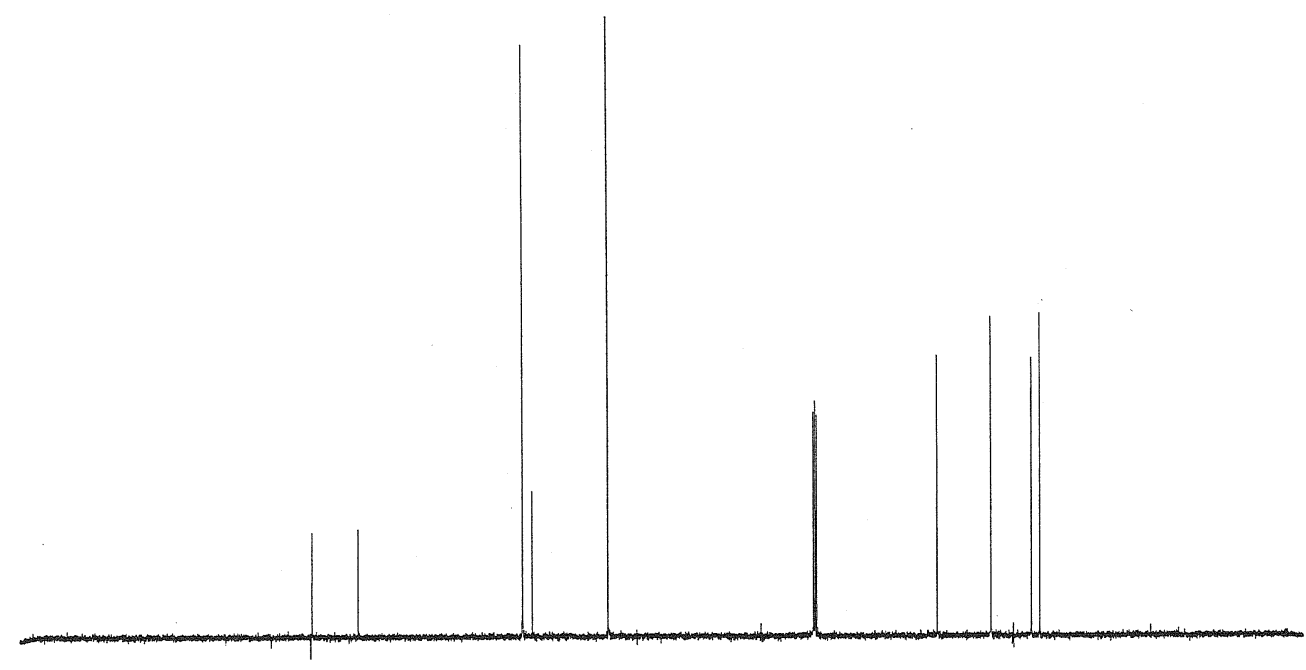

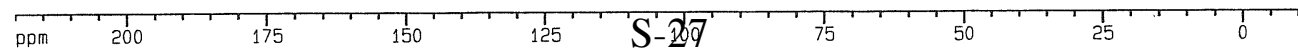

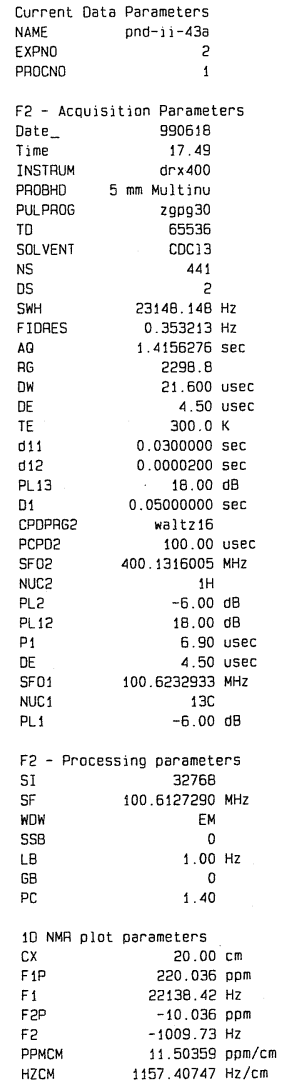



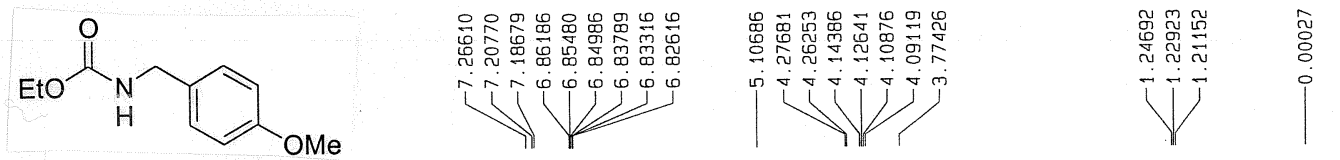

3d

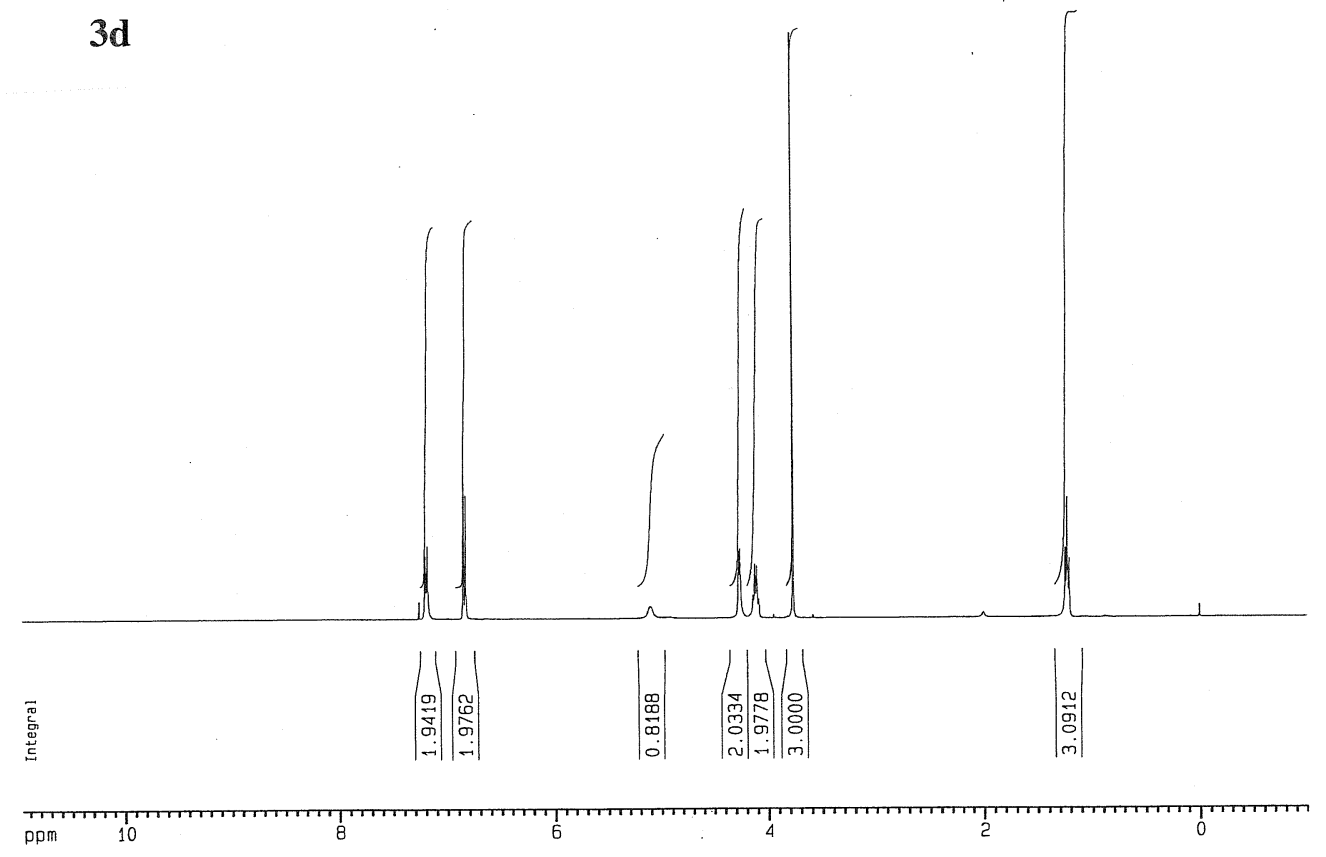

言

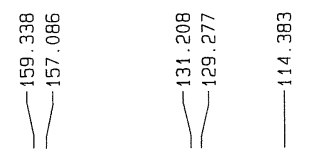

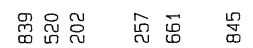

in

ำ 낸

V

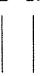

Current Data Parameters

$\begin{array}{lr}\text { NAME } & \text { pnd }-\mathrm{j} \mathrm{j}-43 \mathrm{~b} \\ \text { EXPNO } & 1\end{array}$

PROCN

F2 - Acquisition Parameters

$\begin{array}{lr}\text { Date_ } & 990618 \\ \text { Time } & 18.00\end{array}$

INSTAUM $\quad \mathrm{d} \Gamma \times 400$

5 mm Multinu

$\begin{array}{lr}\text { PULPROG } & 2930 \\ \text { TD } & 32768\end{array}$

$\begin{array}{lr}\text { SOLVENT } & \text { COC13 } \\ \text { NS } & 8\end{array}$

OS $\quad 4789.272 \mathrm{~Hz}$

$4789.146157 \mathrm{~Hz}$
$3.4210291 \mathrm{sec}$

$\begin{array}{lr}A Q & 0.44657 \mathrm{Hec} \\ \mathrm{RG} & 64\end{array}$

104.400 usec

$300.0 \mathrm{~K}$

$1.00000000 \mathrm{sec}$

7.70 usec

4.50 usec
400. $1320007 \mathrm{MHz}$

$1 \mathrm{H}$
$-6.00 \mathrm{~dB}$

PL1 $\quad-6.00 \mathrm{~d}$

F2 - Processing parameters

SI 16384

EM

$E M$
0
0

0

$\begin{array}{lr}\mathrm{GB} & 1.00 \\ \mathrm{PC} & \end{array}$

10 NMB plot parameters

$\begin{array}{lr}\text { CX } & 20.00 \mathrm{~cm} \\ \text { F1P } & 10.968 \mathrm{pD}\end{array}$

F1 $4388.51 \mathrm{~Hz}$

$\begin{array}{ll}F 2 & -1.002 \mathrm{pp} \\ \mathrm{F2} & -400.76 \mathrm{~Hz}\end{array}$
Current Data Parameters

NAME PND-ii-43D

PROCNO ?

F2 - Acquisition Parameters

$\begin{array}{lr} & 990618 \\ \text { Date } & 18.07\end{array}$

$\begin{array}{ll}\text { Time } & 18.07 \\ \text { INSTAUM } & d r \times 400\end{array}$

$5 \mathrm{~mm}$ Multinu

290930

CDC13
SOLVENT

2
W

FIDAES $\quad 0.353213 \mathrm{~Hz}$

1. $4156276 \mathrm{sec}$

21.600 use

4.50 usec

$300.0 \mathrm{~K}$

$0.030000 \mathrm{sec}$

10.00 di

$.05000000 \mathrm{sec}$

waltz 16

100.00 use

$400.1316005 \mathrm{MHz}$

$1 \mathrm{H}$
$-6.00 \mathrm{~dB}$

$18.00 \mathrm{~dB}$

6.90 usec

100. $6232933 \mathrm{MHz}$

$13 \mathrm{C}$
$-6.00 \mathrm{~dB}$

2 - Processing parameters

$\begin{array}{lc}\mathrm{SI} & 32768 \\ \mathrm{SF} & 100.6127290 \mathrm{MHz}\end{array}$

WOW

$\begin{array}{lc}\text { SSB } & 0 \\ \text { LB } & 1.00 \mathrm{HZ} \\ \text { GB } & 0\end{array}$

$\begin{array}{ll}\mathrm{GB} & 1.40 \\ \mathrm{PC} & \end{array}$

10 NMR plot parameters

$\begin{array}{lr}\text { 10 NMA plat parameters } \\ \text { CX } & 20.00 \mathrm{~cm} \\ \text { Fip } & 220.036 \mathrm{ppm}\end{array}$

$\begin{array}{lr}\text { F1P } & 220.036 \mathrm{ppm} \\ \text { F2p } & 22130.42 \mathrm{~Hz} \\ \text { F2P } & -10.036 \mathrm{ppm}\end{array}$

F2 $\quad-1009.73 \mathrm{~Hz}$

$\begin{array}{lr}\text { PPMCM } & 11.50359 \mathrm{ppm} / \mathrm{cm}\end{array}$ 

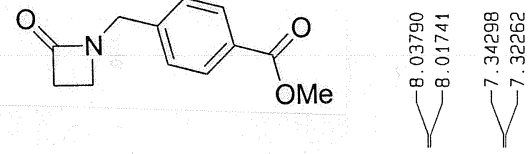

$2 e$

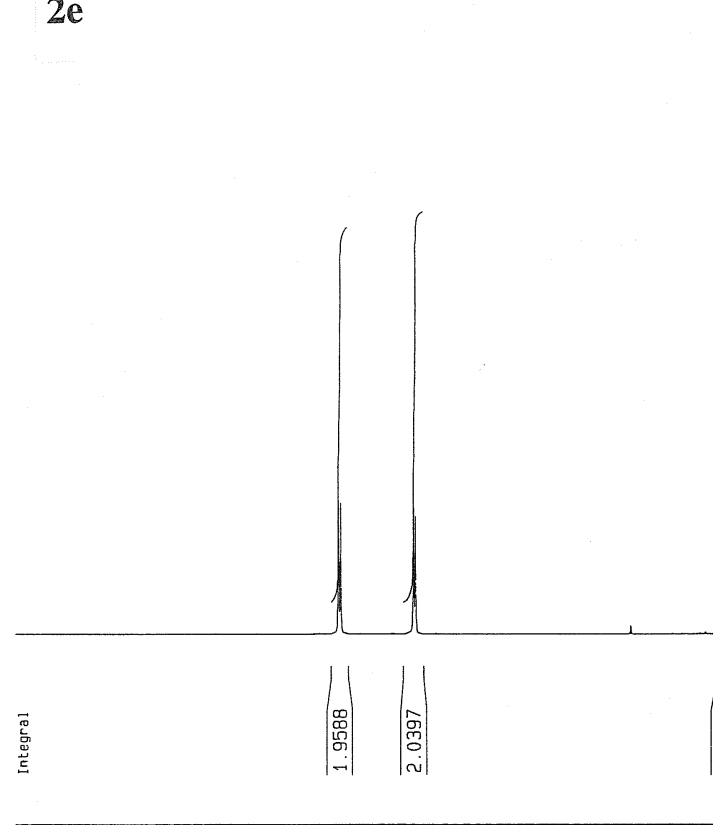

ppm 10

言

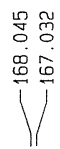

兽 尊䒺兽

$\Xi$ 임요

ن

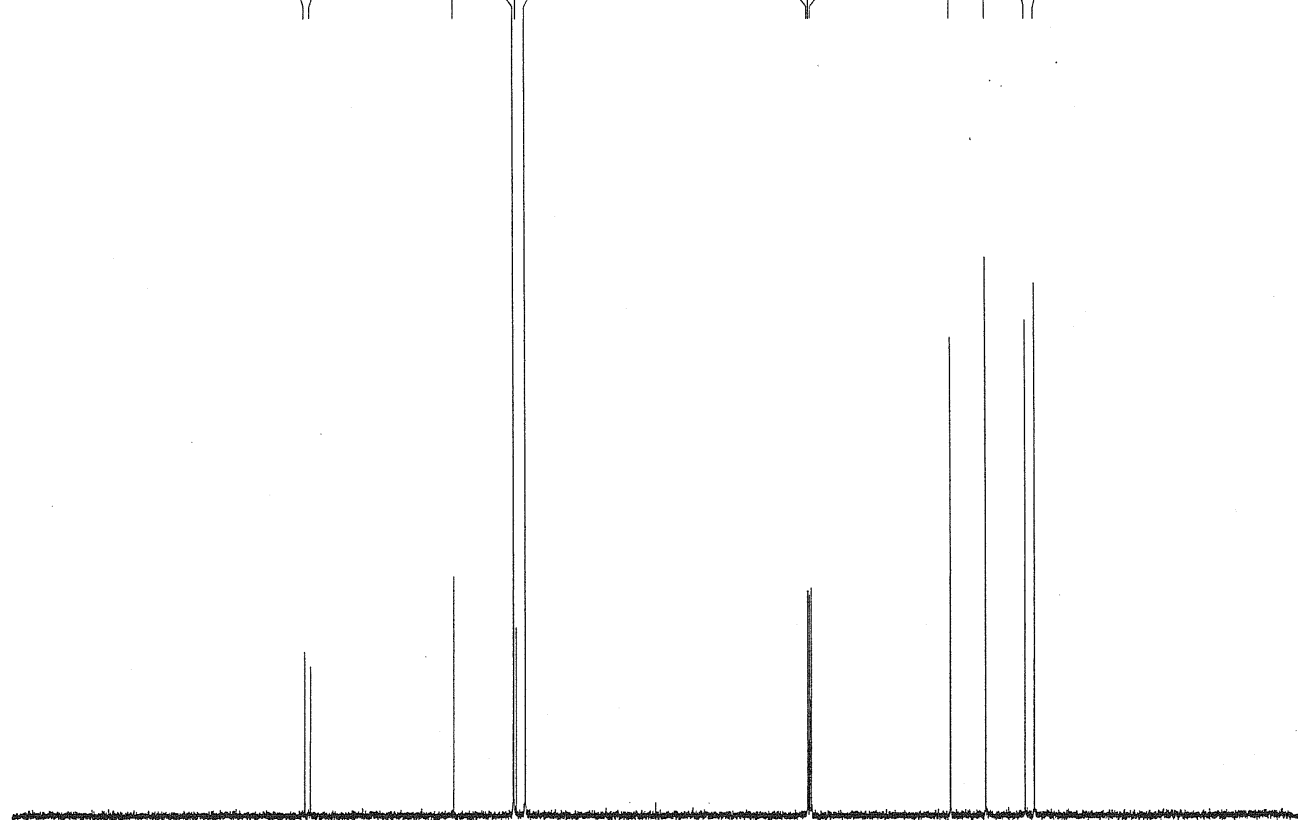

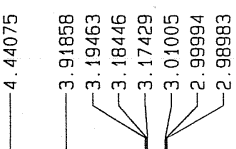

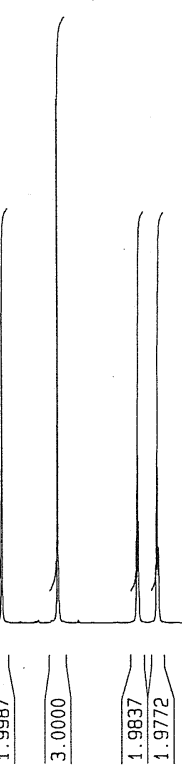

Current Data Parameter

PAOCNO

22 - Acquisition Paraneters

Date-

Time $\quad 15.05$

$\int d r \times 400$

PULPAOG

TO

NS

DS

2930
32768

$\mathrm{CDC} 13$

4789, $272 \mathrm{~Hz}$

$0.146157 \mathrm{~Hz}$

3.4210291 $\mathrm{sec}$

104. 400 use

4.50 use

$300.0 \mathrm{~K}$

$7.70 \mathrm{usec}$
$4.50 \mathrm{sec}$

400.1320007 MHz

$1 \mathrm{H}$
$-6.00 \mathrm{~dB}$

F2 - Processing parameters

$\begin{array}{lr}\text { SI } & 16384 \\ \text { SF } & 400.1299811 \mathrm{MHz}\end{array}$

$E M$
0

$0.30 \mathrm{~Hz}$

1.00

10 NMR plot parameters

$\begin{array}{ll}\mathrm{CX} & 20.00 \mathrm{~cm}\end{array}$

F1 $\quad 4414.23 \mathrm{~Hz}$

$-0.937 \mathrm{pp}$

$59846 \mathrm{pom} / \mathrm{cm}$

$239.46358 \mathrm{~Hz} / \mathrm{cm}$
Current Data Parameters

NAME

PAOCNO

pnd-ii-42a

F2 - Acquisition Parameters

Date_ $\quad 990616$

Time

PROBHD 5 mI MU X $\times 400$

PULPROG

To 29pg30

SOLVENT

DS

SWH

IDRES $\quad 23148.148 \mathrm{~Hz}$

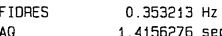

$0.4156276 \mathrm{sec}$
4096

4096
21.600 usec

4.50 usec

$0.0300000 \mathrm{sec}$

0.0000200 sec

$0.05000000 \mathrm{sec}$

100.00 usec
$1315005 \mathrm{MHz}$

$1 \mathrm{H}$

$-6.00 \mathrm{~d}$

5.90 use

100.6232933 use

$13 \mathrm{C}$
$-6.00 \mathrm{~dB}$

PL

F2 - Processing parameter

SI $3276 \mathrm{~B}$

SF

SSB

LB

10 NMR plot parameters

$\begin{array}{ll}\text { CX } & 20.00 \mathrm{~cm} \\ \text { Fip } & 20.036\end{array}$ $22138.42 \mathrm{~Hz}$ $-10.036 \mathrm{ppm}$

$11.50359 \mathrm{ppm} / \mathrm{cm}$ $1157.40747 \mathrm{~Hz} / \mathrm{cm}$ 


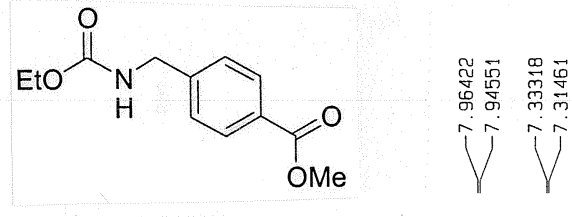

$3 e$
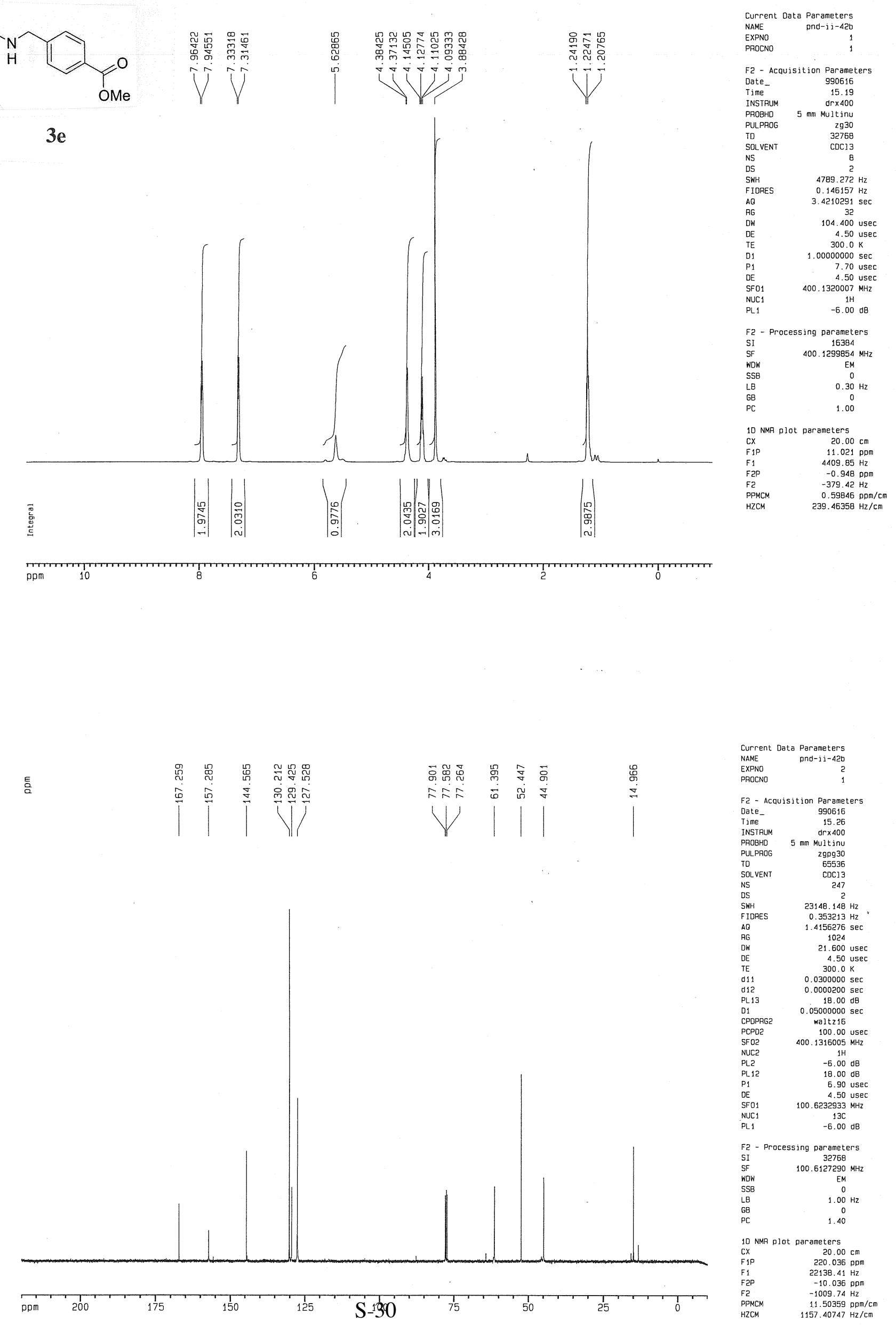


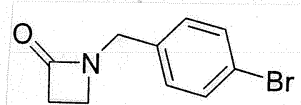

$2 f$
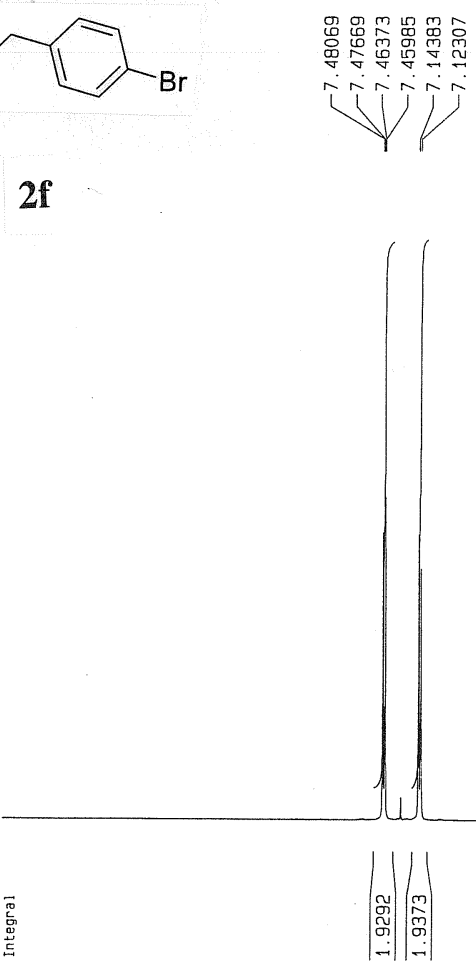
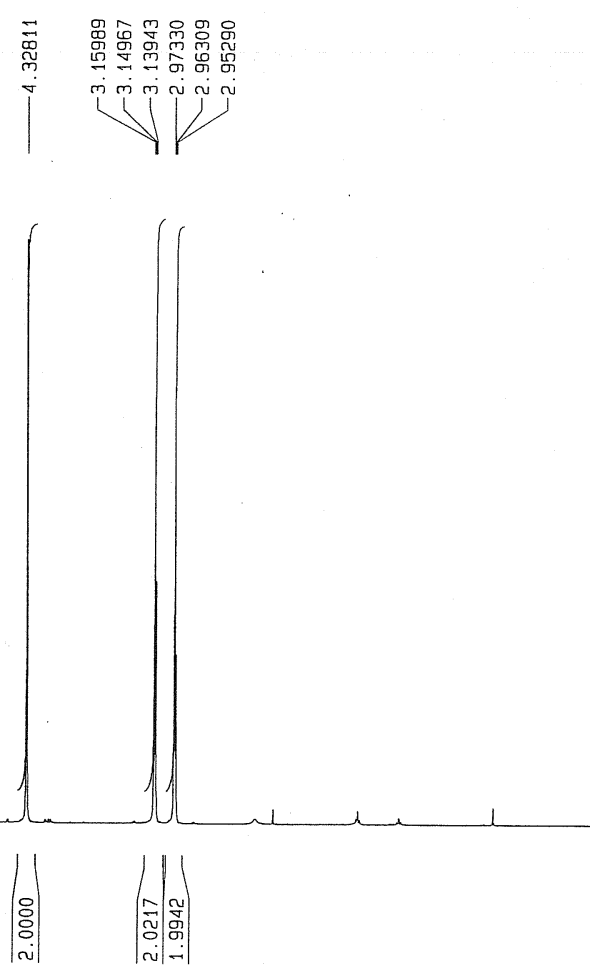

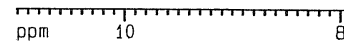

言

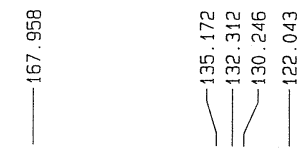

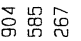

六N

U

惫岸兽

皆界命

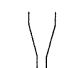

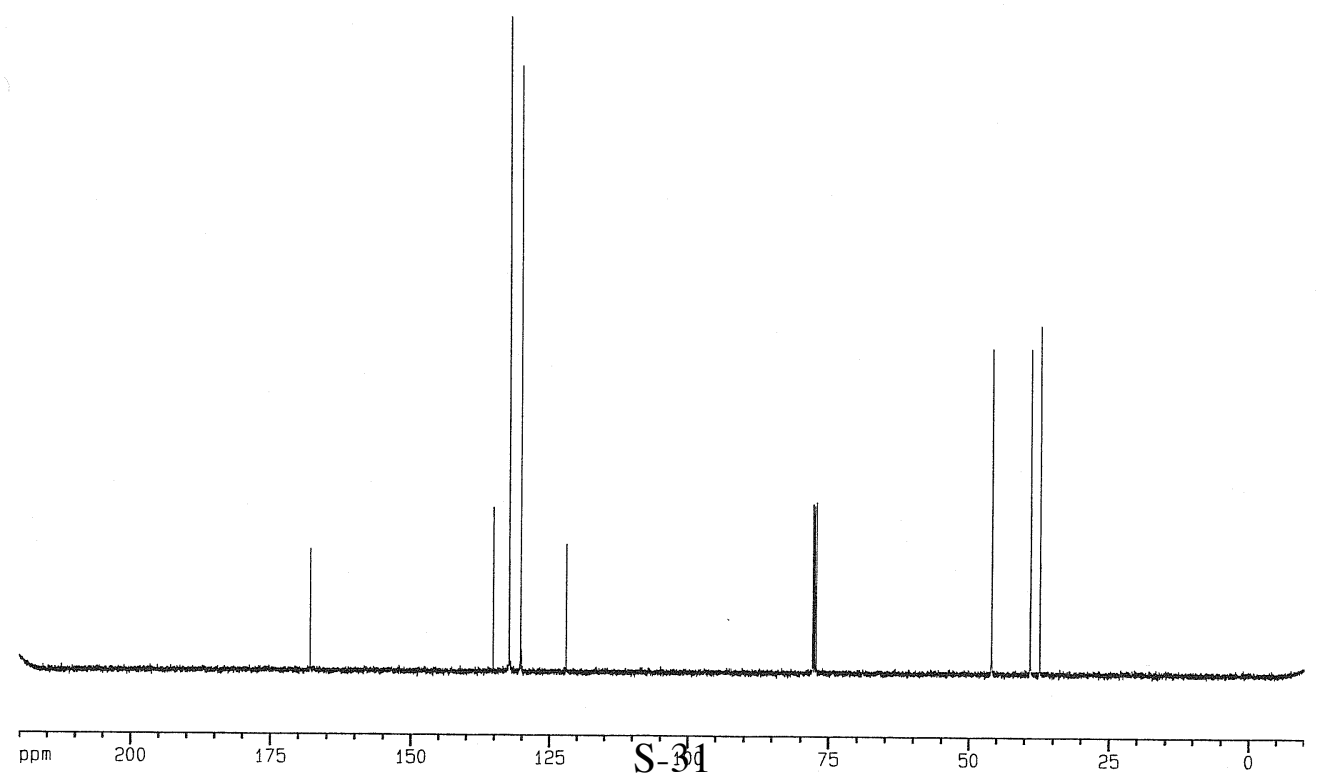

Current Data Parameters

NAME

PAOCNO

F2 - Acquisition Parameters

$\begin{array}{lr}\text { Date } & 990621 \\ \text { Time } & 10.45\end{array}$

10.45
INSTRUM

PAOBHD $5 \mathrm{~mm}$ Multinu

$\begin{array}{lr}\text { PULPROG } & 2930 \\ \text { TD } & 32768\end{array}$

SOLVENT CDC13

$\begin{array}{lr}\text { DS } & 8 \\ \text { SWH } & 2 \\ & 4789.272 \\ \mathrm{FL}\end{array}$

$\begin{array}{ll}\text { SWH } & 4789.272 \mathrm{~Hz} \\ \text { FIDRES } & 0.146157 \mathrm{~Hz}\end{array}$

AB $\quad 3.4210291 \mathrm{sec}$

$\begin{array}{lr}\text { RG } & 64 \\ \text { OW } & 104.400 \text { usec }\end{array}$

4.50 usec

$.00000000 \mathrm{sec}$

7.70 usec

4.50 usec
$400.1320007 \mathrm{MHz}$

$\begin{array}{lr}\text { NUC1 } & 1 \mathrm{H} \\ \text { PL1 } & -6.00 \mathrm{~dB}\end{array}$

F2 - Processing parameters

SI 16384

WOW $\quad 400.1299887$

LB $\quad 0.30 \mathrm{~Hz}$

$\begin{array}{lr}\text { GB } & 0 \\ \mathrm{PC} & 1.00\end{array}$

10 NMR plot parameters

CX $20.00 \mathrm{~cm}$

$\begin{array}{lr}\text { F1P } & 11.013 \mathrm{ppm} \\ \mathrm{F} 1 & 4406.63 \mathrm{~Hz}\end{array}$

$\begin{array}{ll}\text { F2P } & -0.956 \mathrm{ppm} \\ \text { F2 } & -382.64 \mathrm{~Hz}\end{array}$

$\begin{array}{lr}\mathrm{PPMCM} & 0.59846 \mathrm{ppm} / \mathrm{cm} \\ \mathrm{HZCM} & 239.46359 \mathrm{~Hz} / \mathrm{cm}\end{array}$ 

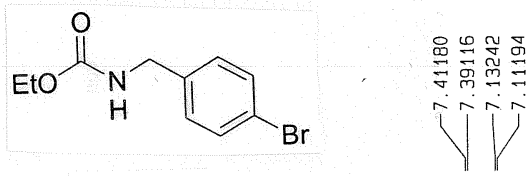

$3 f$
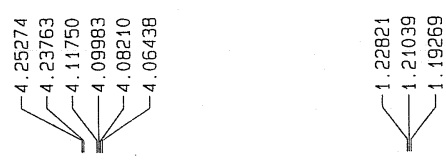

iै।

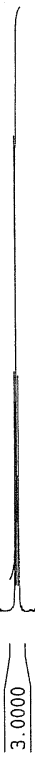

Current Data Parameters

NAME

PROCNO

2 - Acquisition Parameter

Date $\quad 990623$

Time $\quad 11.50$

PROBHD $5 \mathrm{~mm}$ Multinu

PULPAOG $\quad 2930$

$\begin{array}{ll}\text { TD } & 32768 \\ \text { SOLVENT } & \text { CDC13 }\end{array}$

$\begin{array}{lr}\text { NS } & 8 \\ \text { DS } & 2\end{array}$

WHH $4789.272 \mathrm{~Hz}$

3. $4210291 \mathrm{sec}$

104. 400 usec

4.50 usec

$300.0 \mathrm{~K}$

1.00000000 sec

7.70 usec

4.50 usec $0.1320007 \mathrm{MHZ}$ $1 \mathrm{H}$
$-6.00 \mathrm{~dB}$

NUC

PL1

F2 - Processing parameters

$\begin{array}{cc}\text { SI } & 16384 \\ \text { SF } & 400.1299995\end{array}$

WDW

SSB

GB

10 NMR plot parameters

CX $\quad 20.00 \mathrm{~cm}$

F1P $\quad 10.986 \mathrm{ppm}$

$\begin{array}{ll}\text { F1 } & 4395.82 \mathrm{~Hz} \\ \text { F2P } & -0.983 \mathrm{ppm}\end{array}$

$\begin{array}{ll}-0.983 \mathrm{ppm} \\ 2 & -393.46 \mathrm{~Hz}\end{array}$

PPMCM $\quad 0.59846 \mathrm{ppm} / \mathrm{Cm}$

$39.46359 \mathrm{~Hz} / \mathrm{cm}$
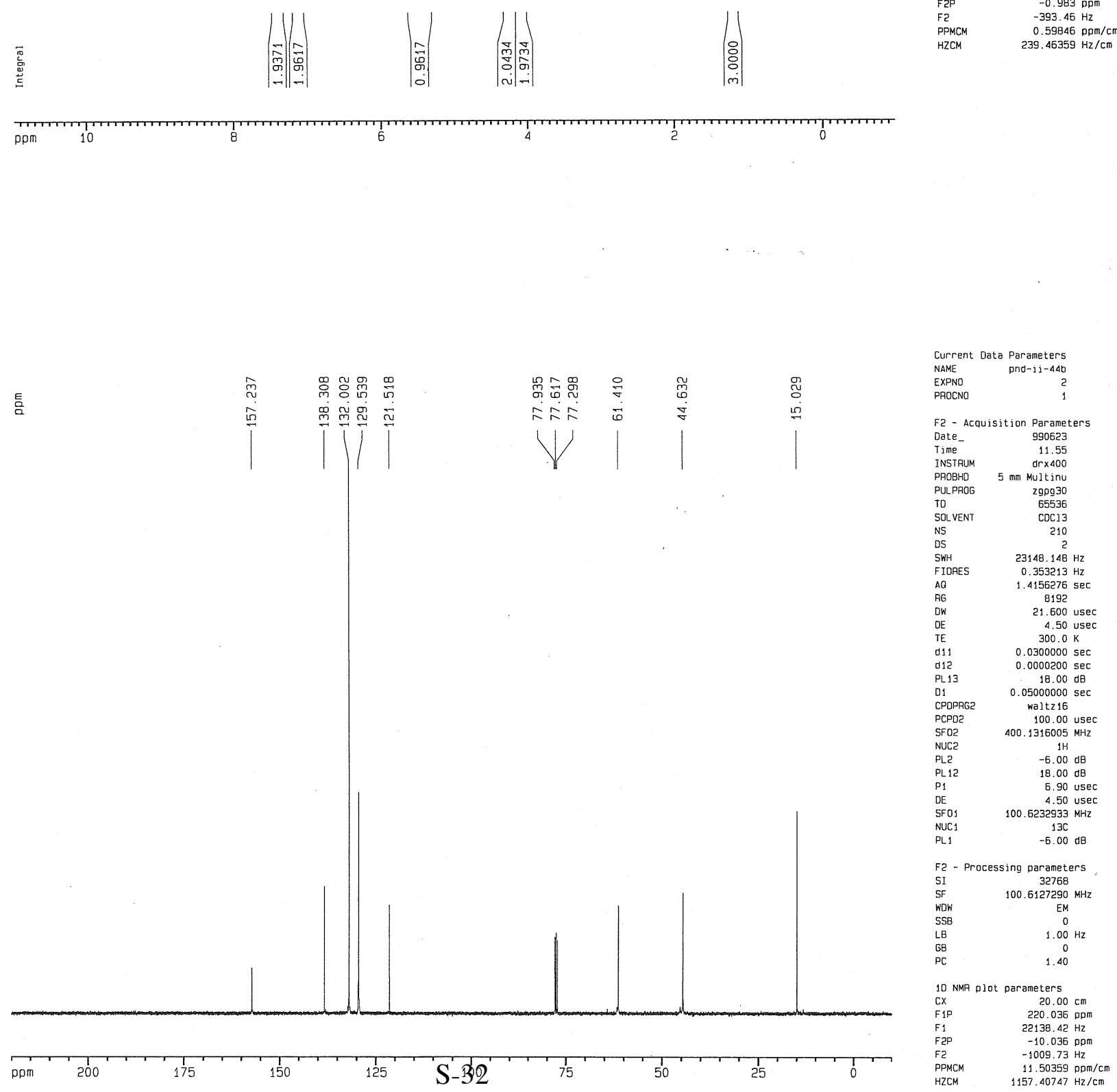

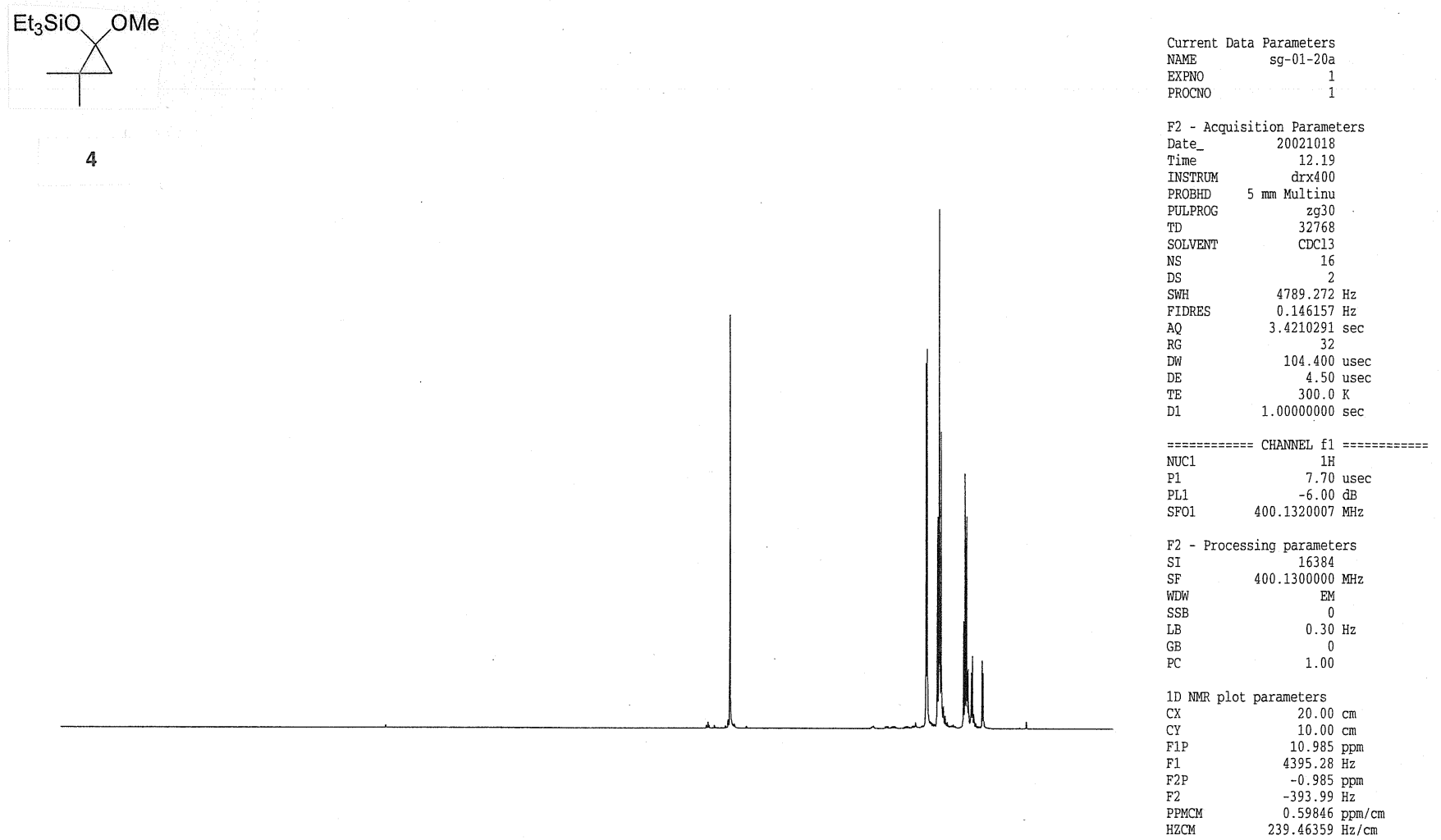

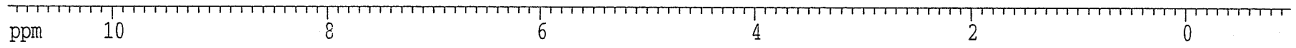

言

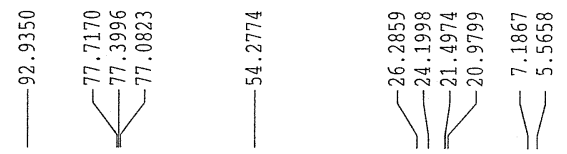

Current Data Parameters

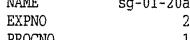

F2 - Acquisition Paraneters

Date_ 20021018

Time-

drx400

inu

$\begin{array}{lr}\text { PULPROG } & \text { 2gpg30 } \\ \text { TD } & 65536\end{array}$

$\begin{array}{lr}\text { TD } & 65536 \\ \text { SOLVENT } & \text { CDC13 } \\ \text { NS } & 501 \\ \text { DS } & 2\end{array}$

$\begin{array}{lr}\text { DS } & 2 \\ \text { SWH } & 23148.148 \mathrm{~Hz} \\ \text { PTDES } & 0.353213 \mathrm{~Hz}\end{array}$

FIDRES
AQ

21.600 usec
4.50 usec

$300.0 \mathrm{~K}$

$0.05000000 \mathrm{sec}$

$0.03000000 \mathrm{sec}$

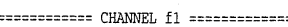

NUC1 $13 \mathrm{C}$

PL1 $\quad 2.00 \mathrm{~dB}$

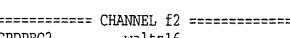

$\begin{array}{ll}\text { CDPPRG2 } & \text { Waltz16 } \\ \text { NUC2 } & \text { wall }\end{array}$

$\begin{array}{lc}\text { NUC2 } & 1 \mathrm{H} \\ \text { PCPD2 } & 100.00 \text { us }\end{array}$

$\begin{array}{lr}\text { PCPD2 } & 100.00 \mathrm{us} \\ \text { PL2 } & 0.00 \mathrm{~dB} \\ \text { PL12 } & 18.00 \mathrm{~dB}\end{array}$

$\begin{array}{lr}\text { PL13 } & 18.00 \mathrm{~dB} \\ \text { SF02 } & 400.1316005 \mathrm{MHz}\end{array}$

$\begin{array}{lr}\text { F2 } & \text { - Processing parameters } \\ \text { SI } & 32768 \\ \text { SF } & 100.6127290 \mathrm{MHz}\end{array}$

$\begin{array}{lc}\text { WDW } & 100.6127 \\ \text { WDW } & \text { EM } \\ \text { SSB } & 0 \\ \text { LB } & 1.00 \mathrm{~Hz}\end{array}$

$\begin{array}{lr}\text { LB } & 1.00 \\ \text { GB } & 0\end{array}$

1D NMR plot parameters

$\begin{array}{lr}\text { 1D NMR plot parameters } \\ \text { CX } & 20.00 \mathrm{~cm} \\ \text { CY } & 12.00 \mathrm{~cm} \\ \text { F1P } & 215.000 \mathrm{ppm} \\ \text { F1 } & 21631.74 \mathrm{~Hz} \\ \text { F2P } & -5.000 \mathrm{ppm} \\ \text { F2 } & -503.06 \mathrm{~Hz} \\ \text { PPMCM } & 11.00000 \mathrm{ppm} / \mathrm{cm} \\ \text { HZCM } & 1106.73999 \mathrm{~Hz} / \mathrm{cm}\end{array}$



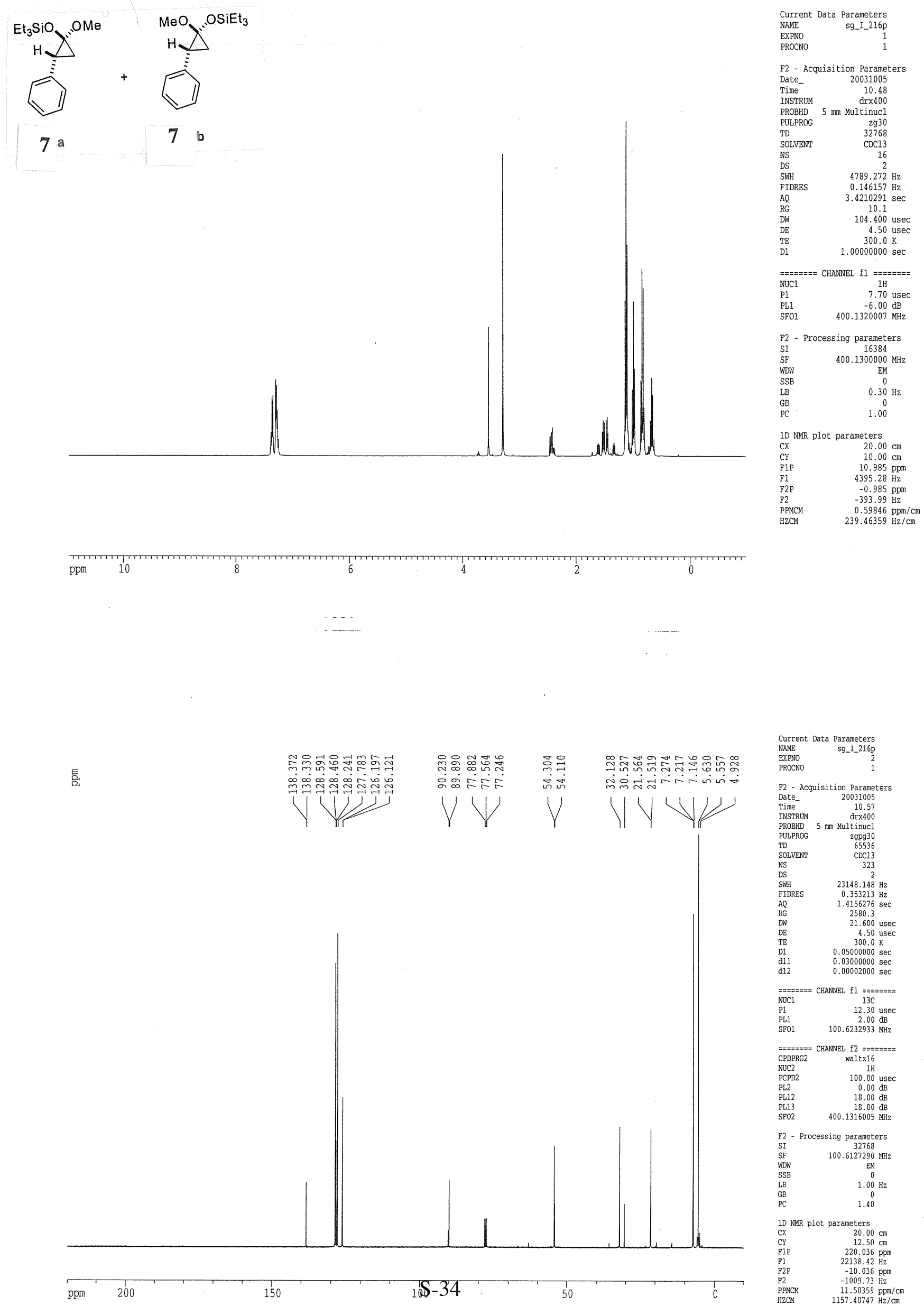


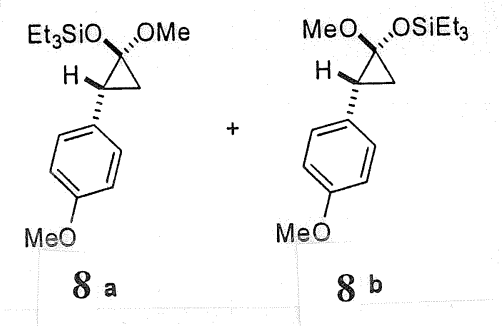

ppm

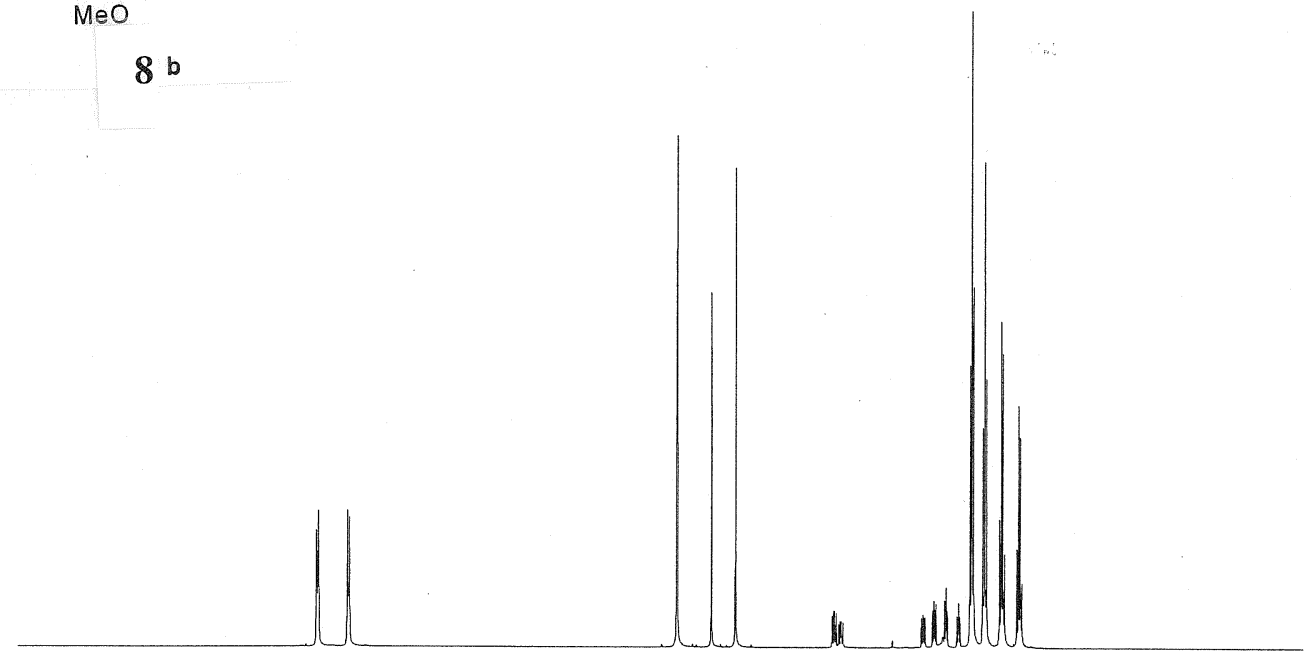

${ }_{8}$

Tा1'
T11
爱恋

ํํำ

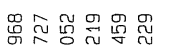

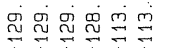

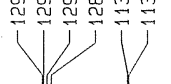

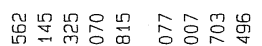

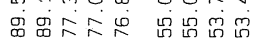

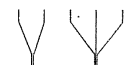

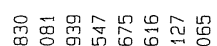

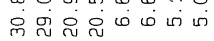

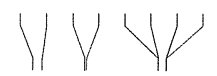

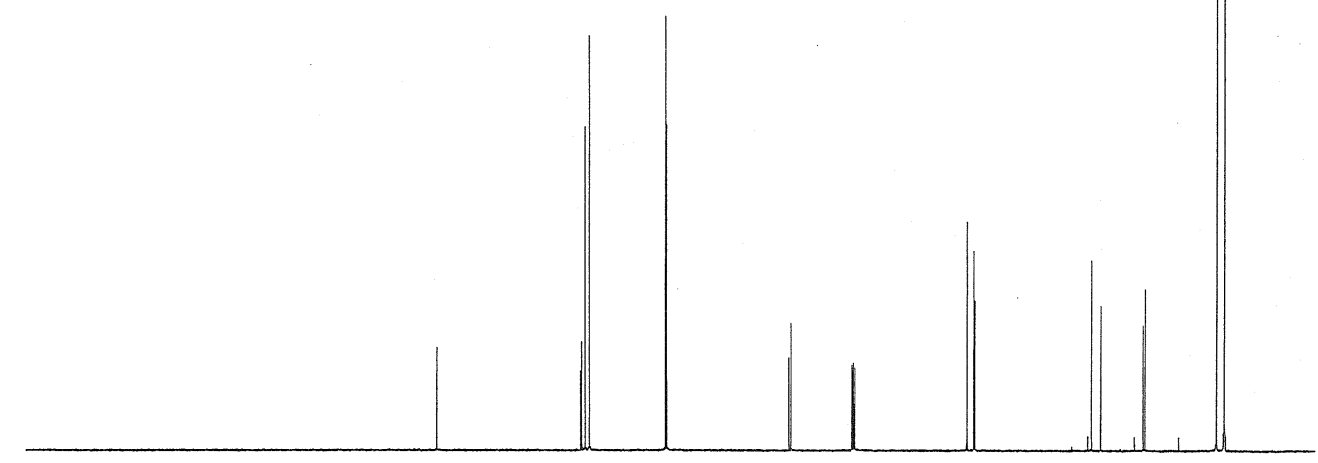

ppm S $-35^{\circ}$
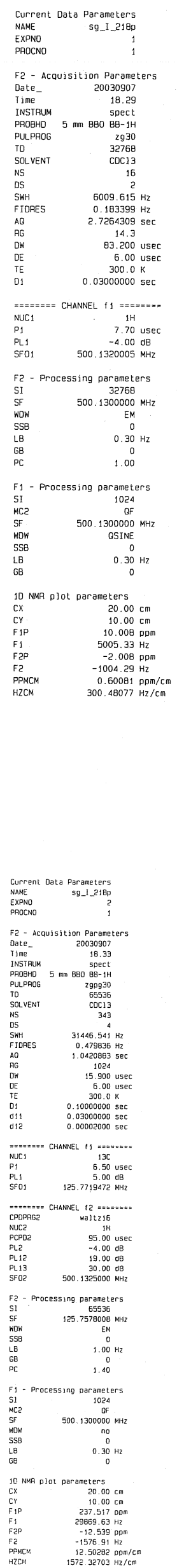


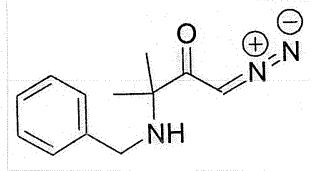

$5 a$

52

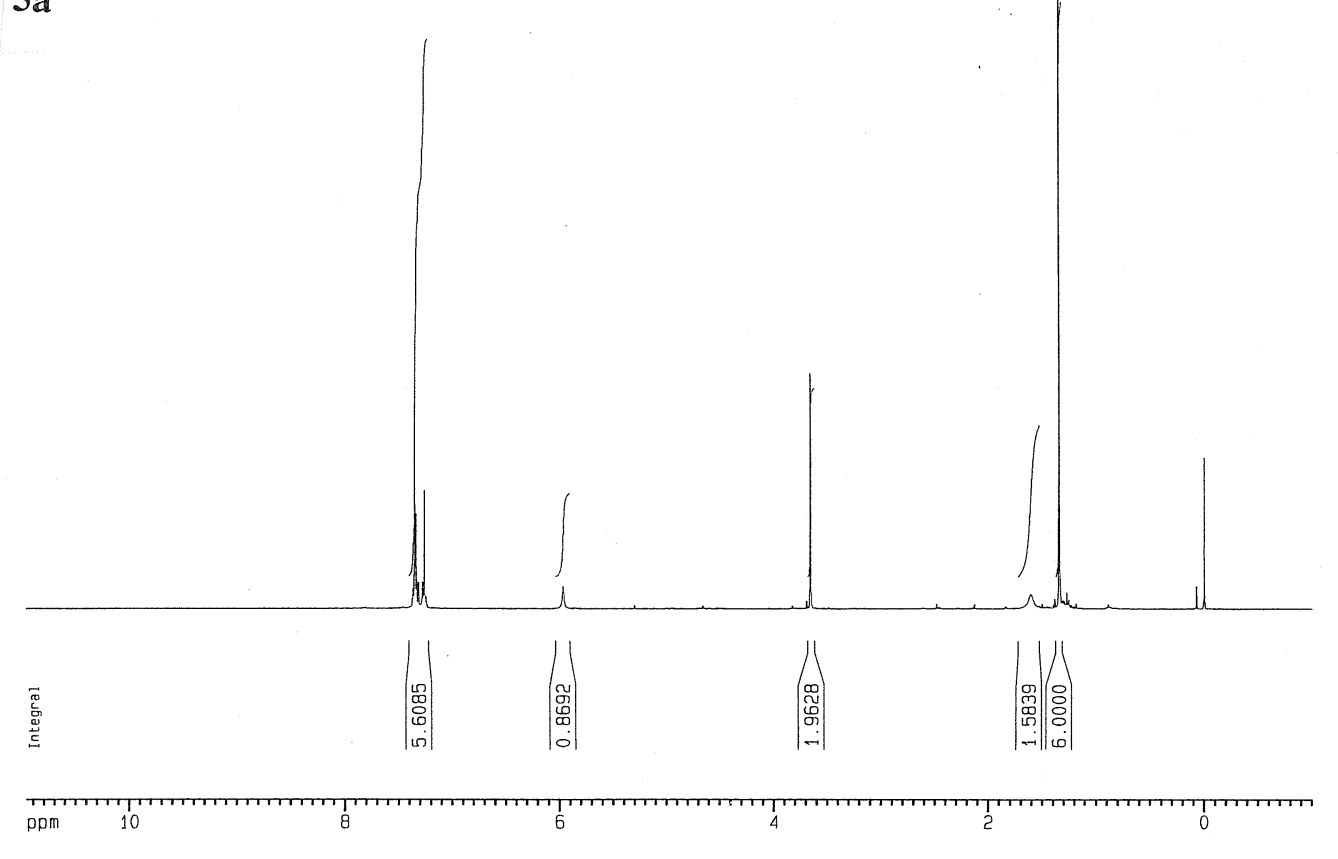

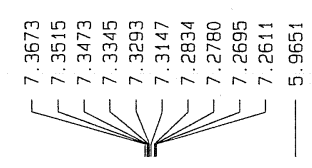

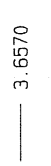

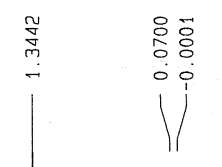

Current Data Parameters

NAME DNd-ii-128a

EXPNO

F2 - Acquisition Parameters

Date $\quad 991110$

$\begin{array}{lr}\text { Time } & 15.03 \\ \text { INSTRUM } & d r \times 400\end{array}$

PAOBHD $5 \mathrm{~mm}$ Multinu

$\begin{array}{lr}\text { PULPAOG } & 2930 \\ \text { TD } & 32768\end{array}$

TD

NS

DS

2
2

$0.146157 \mathrm{~Hz}$
HDRES

3. $4210291 \mathrm{sec}$

512
104.400 usec

4.50 usec
usec

$.00000000 \mathrm{sec}$

7.70 use

7.70 usec
4.50 usec

400. $1320007 \mathrm{MHz}$

$1 \mathrm{H}$
$-6.00 \mathrm{OB}$

PL1

F2 - Processing parameters

SI Processing parameters

$\begin{array}{lc}\text { SF } & 400.1300091 \mathrm{MHz} \\ \text { WOW } & \text { EM }\end{array}$

$\begin{array}{lc}\text { SSB } & 0 \\ . B & 0.30 \mathrm{~Hz}\end{array}$

$\begin{array}{rr}\mathrm{Ge} & 0 \\ \mathrm{PC} & 1.00\end{array}$

10 NMR plot parameters

$\begin{array}{lr}\text { CX } & 20.00 \mathrm{~cm} \\ \text { F1P } & 10.962 \mathrm{pom}\end{array}$

$10.962 \mathrm{ppm}$
$4386.17 \mathrm{~Hz}$

$-1.007 \mathrm{ppm}$

$-1.007 \mathrm{ppm}$
$-403.10 \mathrm{~Hz}$

$\begin{array}{ll}\text { PPMCM } & 0.59846 \mathrm{ppm} / \mathrm{cm} \\ \mathrm{HZCM} & 239.46359 \mathrm{~Hz} / \mathrm{cm}\end{array}$

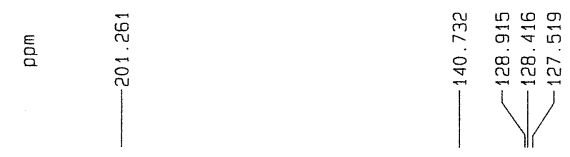

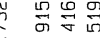

|

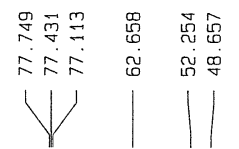

กลก

品

กั

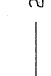

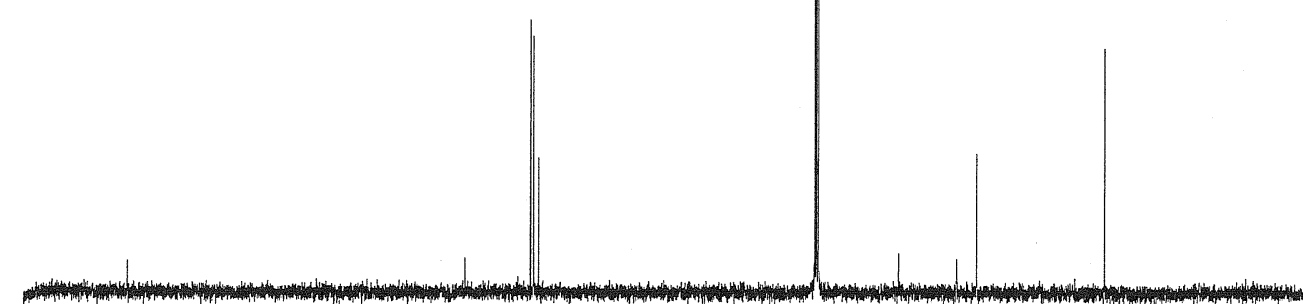

$\frac{1}{50}$

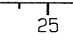

150 


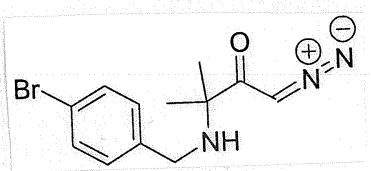

$5 b$
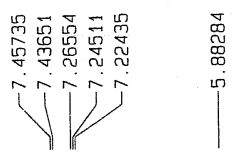

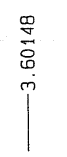

岕
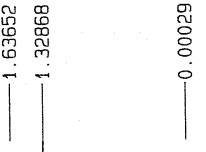

Current Data Parameters

EXPNO $\quad 1$

F2 - Acquisition Parameters

Date_ 991109

Time $\quad 16.34$

INSTAUM $\quad$ dr $\times 400$

5 mm Multinu
PULPRDOG
2930

$\begin{array}{ll}\text { TD } & 32768 \\ \text { SOLVENT } & \mathrm{COC} 13\end{array}$

SWH

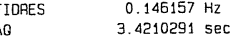

3. $4210291 \mathrm{sec}$

181
104.400 usec

4.50 usec

$1.00000000 \mathrm{sec}$

$7.70 \mathrm{usec}$
$4.50 \mathrm{usec}$

4.50 usec

$400.1320007 \mathrm{MHz}$

$-6.00 \mathrm{~dB}$

PL1

F2 - Processing parameters

SI 16384

$\begin{array}{lr}\text { SF } & 400.1300074 \\ \text { WDW } & \text { EM }\end{array}$

$\begin{array}{lc}\text { SSB } & 0 \\ \text { LB } & 0.30 \mathrm{~Hz} \\ \text { GB } & 0\end{array}$

GB

10 NMF plot parameters

$\begin{array}{lr}\text { CX } & 20.00 \mathrm{~cm} \\ \text { F1P } & 10.966 \mathrm{pp}\end{array}$

F1 $4387.93 \mathrm{~Hz}$

$\begin{array}{ll}\text { F2P } & -1.003 \mathrm{ppm} \\ \text { F2 } & -401.35 \mathrm{~Hz}\end{array}$

$\begin{array}{lr}\text { PPMCM } & 0.59846 \mathrm{ppm} / \mathrm{cm} \\ \text { HZCM } & 239.46359 \mathrm{~Hz} / \mathrm{cm}\end{array}$
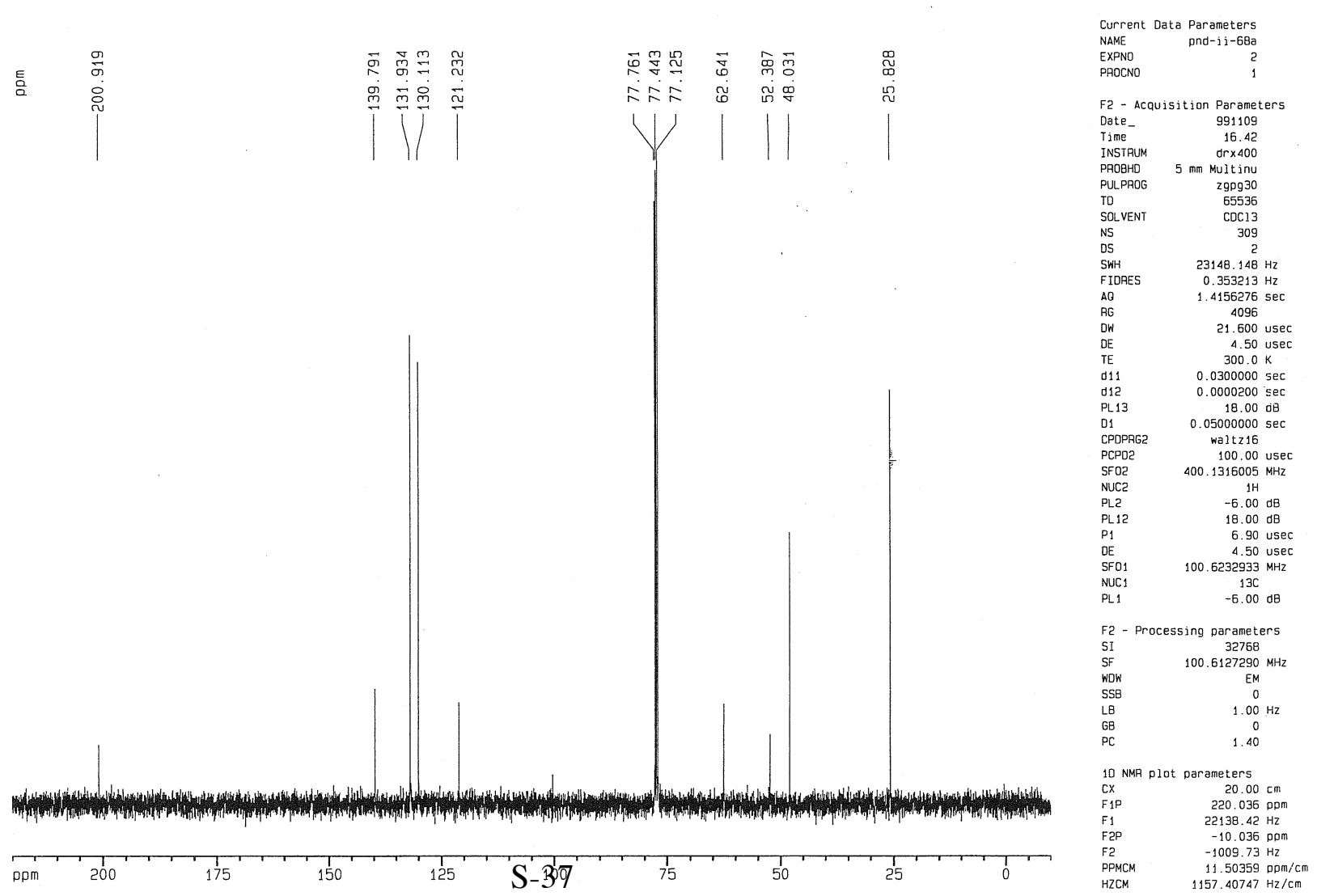
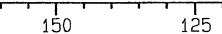

S-30
4

. 
<smiles>COC(=O)c1ccc(CNC(C)(C)C(=O)C=[N+]([O-])[O-])cc1</smiles>

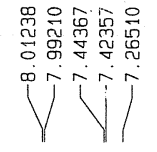

$5 c$

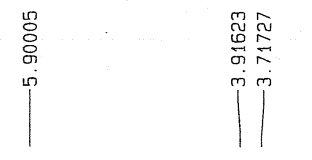

Current Data Parameters

EXPNO

1

F2 - Acciuisition Parameter

Dete- $\quad 991108$

$\begin{array}{lr}11.50 \\ \text { INSTRUM } & \text { or } \times 400\end{array}$

PROBHD $5 \mathrm{~mm}$ Multinu

$\begin{array}{lr}\text { PULPROG } & 2930 \\ \text { TD } & 32768 \\ \text { SOLVENT } & \text { CDC } 13\end{array}$

NS

SWh

FIDRES $\quad 4789.272 \mathrm{~Hz}$

$\begin{array}{ll}A Q & 0.146157 \mathrm{~Hz} \\ A \mathrm{~N} & 3.4210291 \mathrm{sec}\end{array}$

$\begin{array}{ll}\text { RG } & 362 \\ \text { DW } & 104.400 \text { usec } \\ \text { DE } & 4.50 \text { use }\end{array}$

4.50 use

$1,00000000 \mathrm{sec}$

7.70 usec

$4.50 \mathrm{usec}$
$400.1320007 \mathrm{MHz}$

$-6.00 \mathrm{~dB}$

NUL1

F2 - Processing parameters

$\begin{array}{lr}\text { SI } & 16384 \\ \text { SF } & 400.1300077 \mathrm{MHZ}\end{array}$

WOW
SSB
EM

$\begin{array}{lc}\text { LB } & 0 \\ G B & 0.30 \mathrm{~Hz}\end{array}$

10 NMP plot parameters

CX $\quad 20.00 \mathrm{~cm}$

$\begin{array}{ll}\text { F1P } & 10.966 \mathrm{ppm}\end{array}$

F2P $\quad-4387.63 \mathrm{~Hz}$

F2 $-401.64 \mathrm{~Hz}$

$\begin{array}{lr}\text { PPMCM } & 0.59846 \mathrm{ppm} / \mathrm{cm} \\ \mathrm{HZCM} & 239.46359 \mathrm{~Hz} / \mathrm{cm}\end{array}$

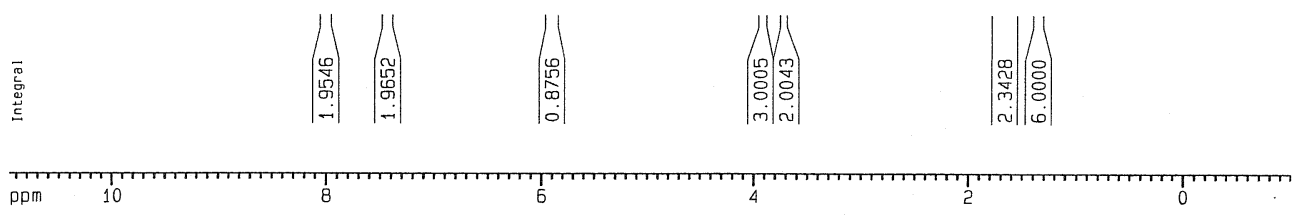

$239.46359 \mathrm{~Hz} / \mathrm{cm}$
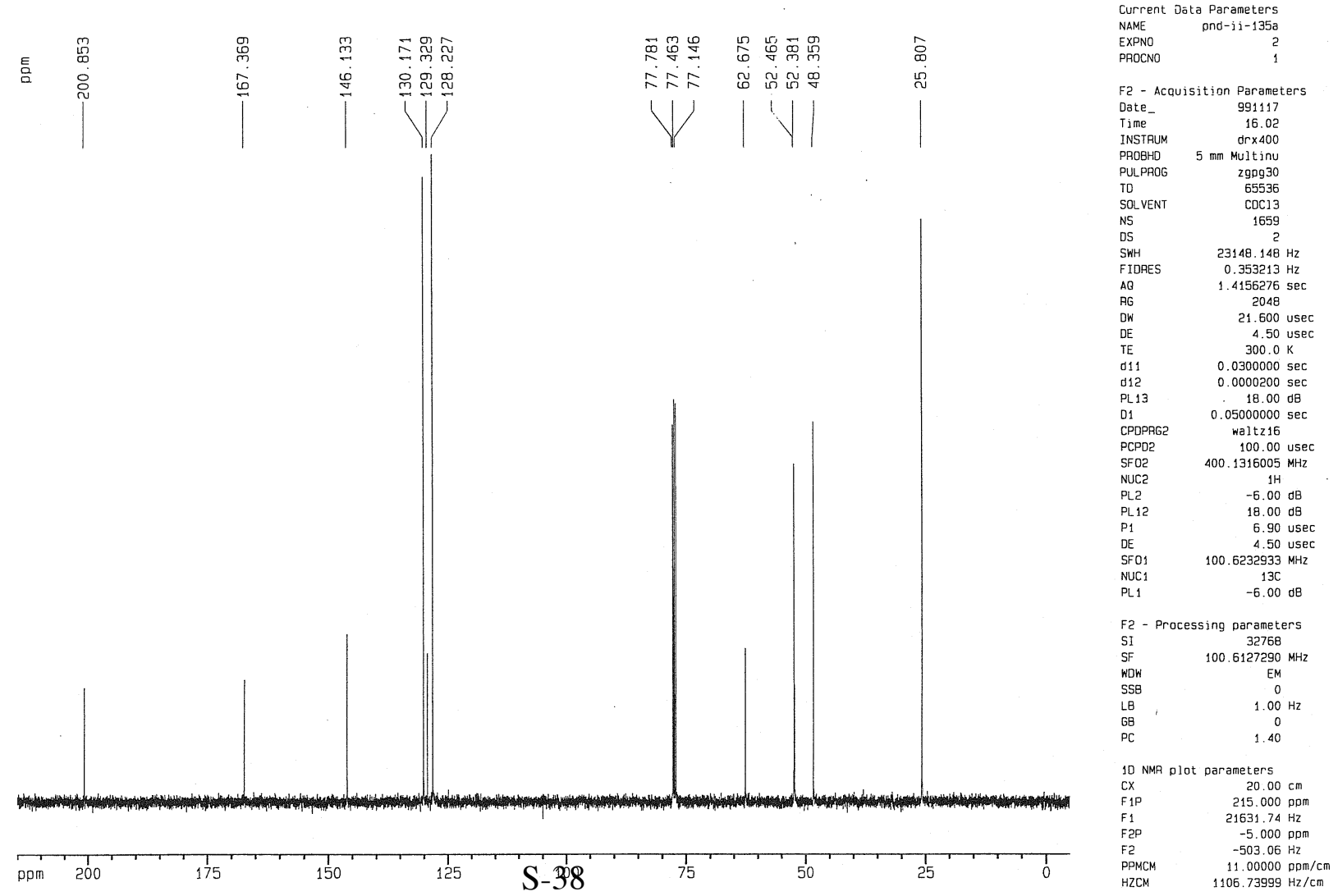

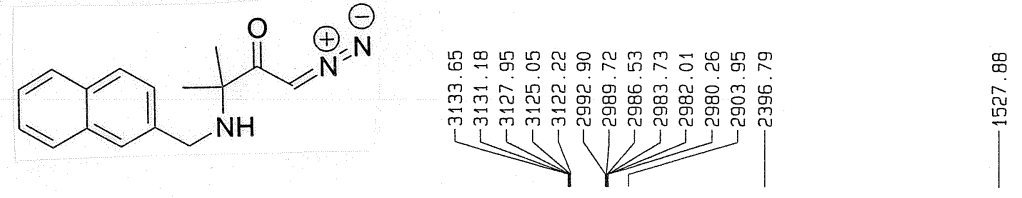

욤

Current Data Parameter

NAME

EXPNO

F2 - Acquisition Parameters

Date_ $\quad 991109$

$\begin{array}{ll}\text { Time } & 16.47\end{array}$

PROBHD $5 \mathrm{~mm}$ Multinu

$5 d$

SOLVEN

SWH

AQ

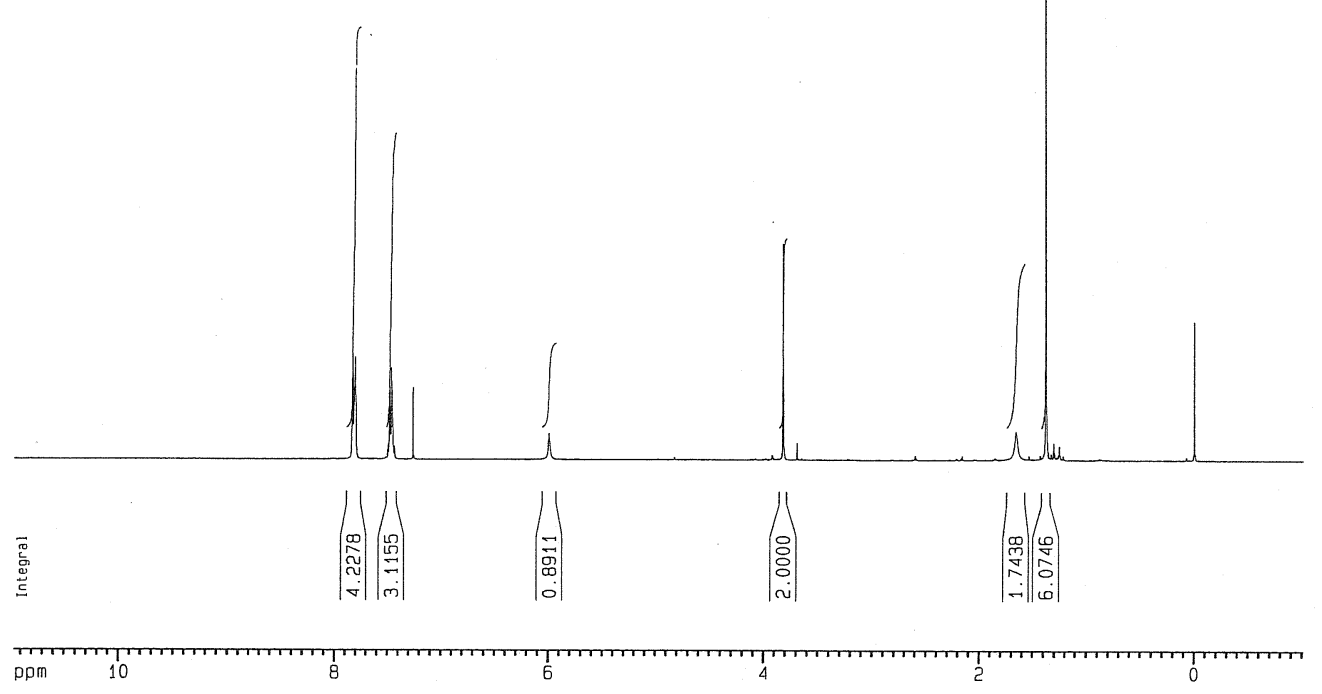

$3.4210291 \mathrm{sec}$
256

104.400 usec
DE 4.50 usec

$1.00000000 \mathrm{sec}$

7.70 usec
4.50 usec

SF01 $\quad 400.1320007 \mathrm{MHz}$

$\begin{array}{ll}\text { NUC1 } & 1 \mathrm{H} \\ \text { PL1 } & -6.00 \mathrm{~dB}\end{array}$

F2 - Processing parameters

$\begin{array}{lc}\text { SI } & 16384 \\ \text { SF } & 400.1300106 \mathrm{MHz}\end{array}$

WDW
SSB

GB

10 NMR plot parameters

$\begin{array}{ll}\text { CX } & 20.00 \mathrm{~cm}\end{array}$

$\begin{array}{lr}\text { F1P } & 10.958 \mathrm{ppm} \\ \text { F1 } & 4384.71 \mathrm{~Hz}\end{array}$

$\begin{array}{ll}F 2 P & -1.011 \mathrm{ppm} \\ \text { F2 } & -404.56 \mathrm{~Hz}\end{array}$

PPMCM $\quad-404.56 \mathrm{~Hz}$

$\mathrm{HZCM}$

$239.46359 \mathrm{~Hz} / \mathrm{cm}$ $0.0300000 \mathrm{sec}$

$\begin{array}{ll}\mathrm{d} 12 & 0.0000200 \mathrm{sec} \\ \text { PL13 } & 18.00 \mathrm{~dB}\end{array}$

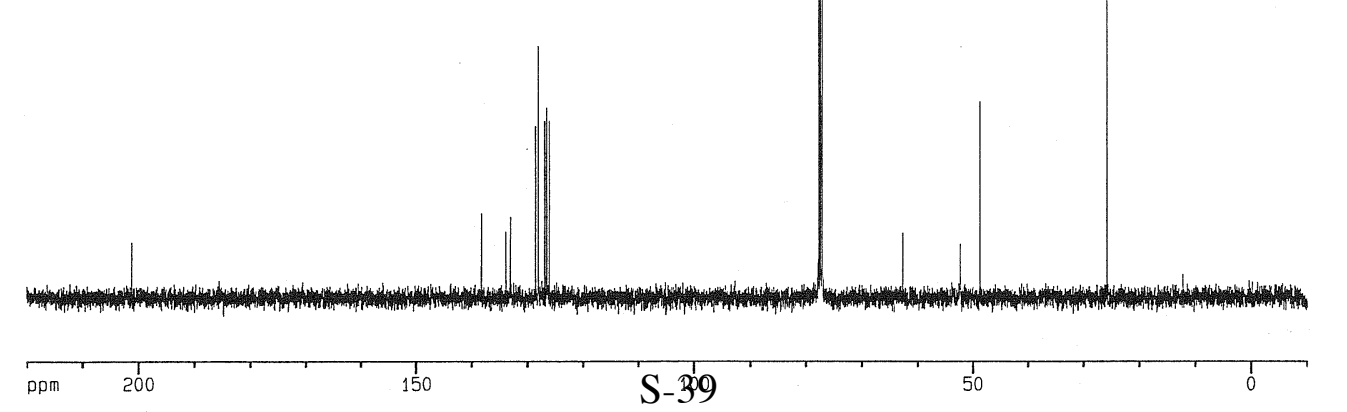

F2 - Processing parameters

SI 32768

$\begin{array}{lr}\text { SF } & 100.6127290 \\ \text { WDW } & \text { EM }\end{array}$ 


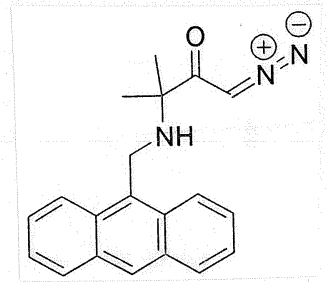

$5 e$

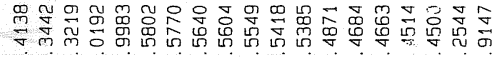

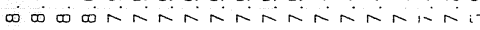

$\underbrace{L L L L S}_{71}+1$

\section{怘 \\ 1}

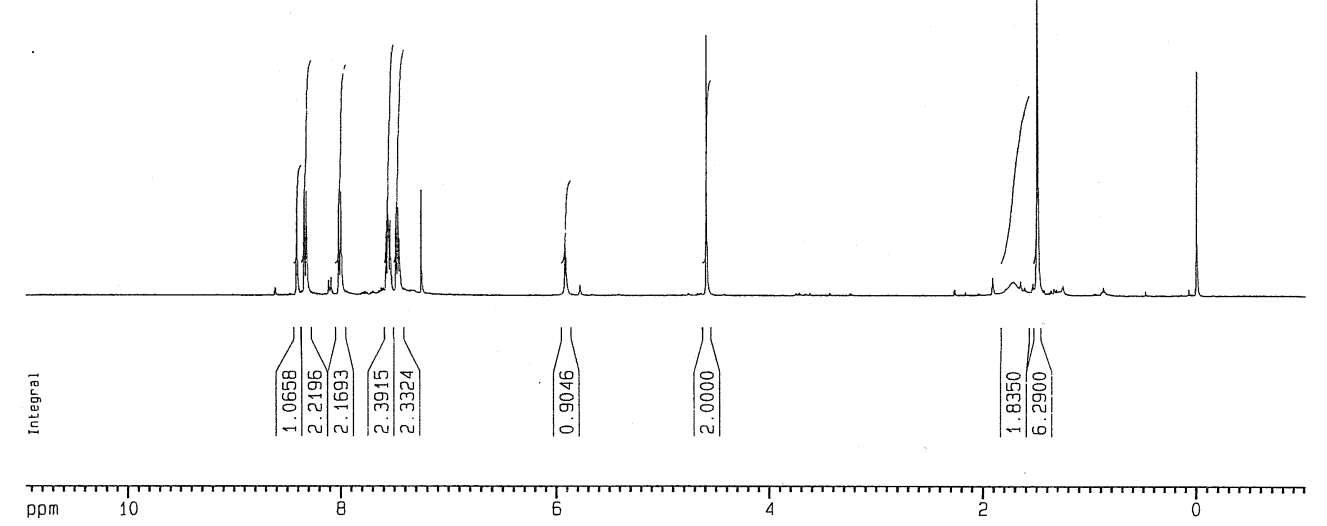

틈

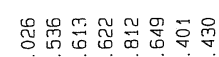

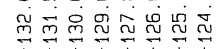

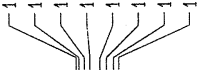

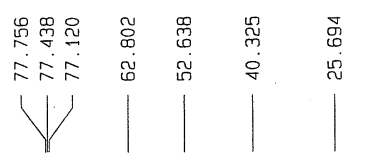

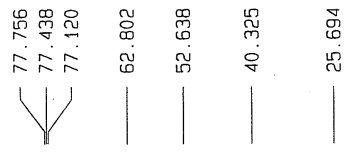
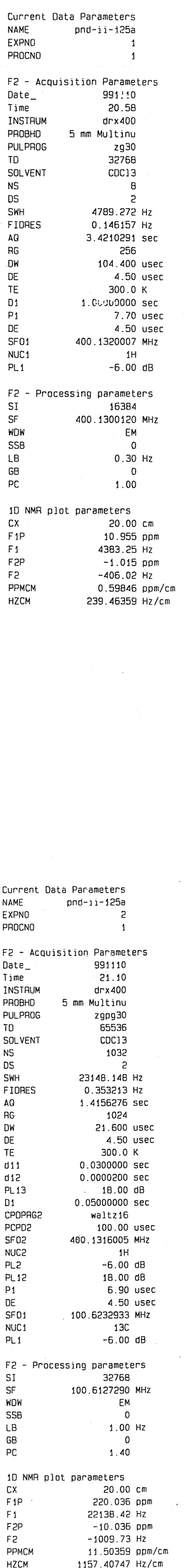


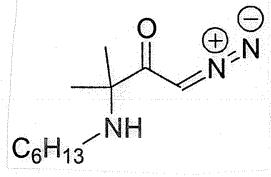

5f

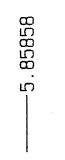

|
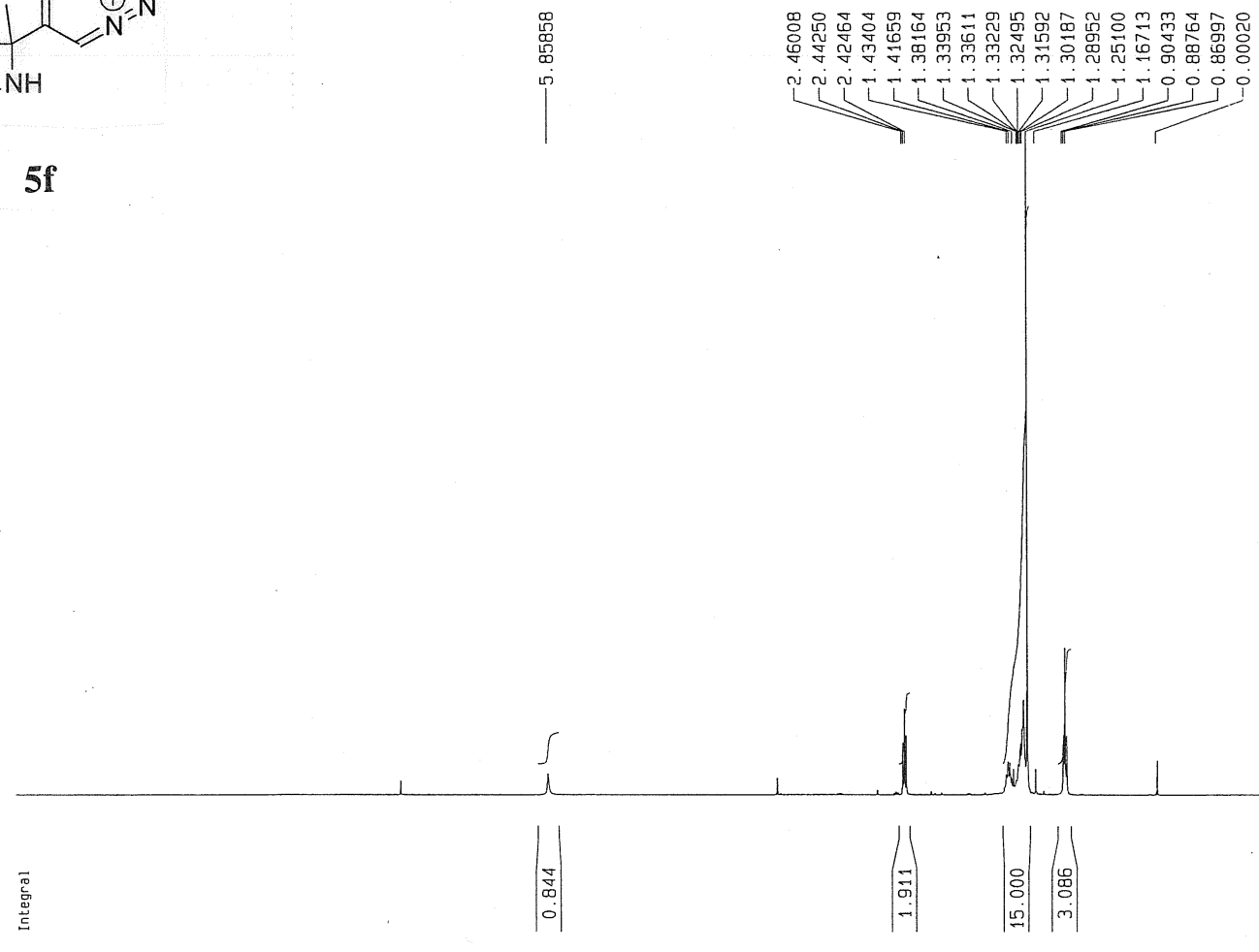

ppm 10

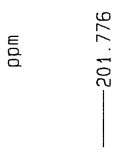

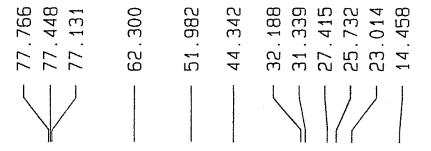

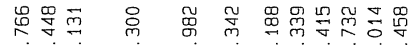

$\left.\sqrt{1}\right|_{1}|1| 1 \mid$

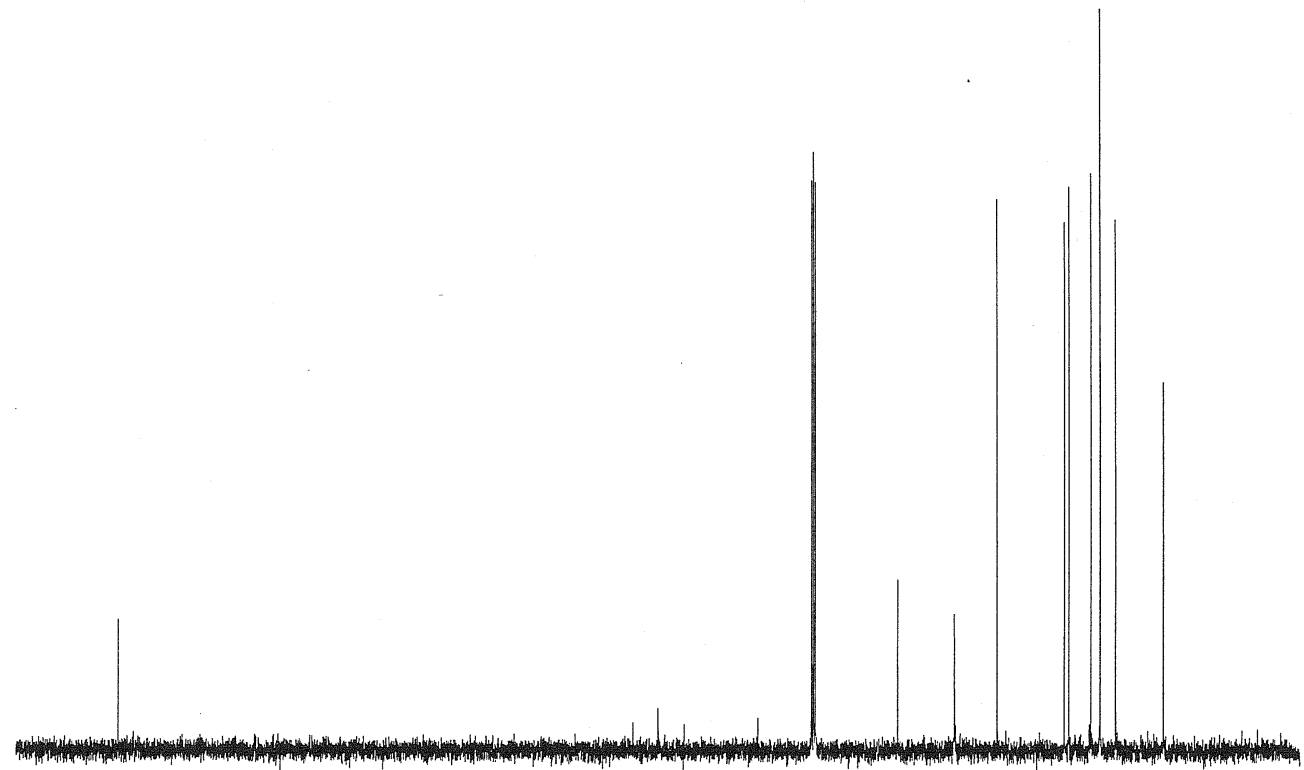

ppm

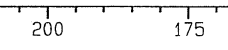

175

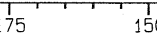

${ }_{150}^{1}$

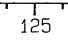

Current Data Parameters

NAME pnd-1i-35a

EXPNO
PROCNO

F2 - Acquisition Parameters

Date $\quad 991110$

Time $\quad 14.51$

INSTRUM dr $\quad$ X400 PROBHD $5 \mathrm{~mm}$ Multinu $\begin{array}{lr}29 p 930 \\ \text { TD } & 65336\end{array}$

$\begin{array}{lr}\text { SOLVENT } & \text { CDC13 } \\ \text { NS } & 504 \\ \text { DS } & 5\end{array}$

DS
SWH
$23148.148 \mathrm{~Hz}$

FIDRES $\quad 0.353213 \mathrm{~Hz}$

$A Q \quad 1.4156276 \mathrm{sec}$

$\begin{array}{ll}\text { AG } & 4096 \\ \text { OW } & 21.600 \text { usec }\end{array}$

DE 4.50 usec

$\begin{array}{ll}\text { TE } & 300.0 \mathrm{~K} \\ \text { O11 } & 0.0300000 \mathrm{sec}\end{array}$

$12 \quad 0.0000200 \mathrm{sec}$

$\begin{array}{ll}\text { PL13 } & 18.00 \mathrm{~dB}\end{array}$

0.05000000
PDPAG

\begin{tabular}{lr} 
PCPD2 & 100.00 usec \\
\hline SPO2 & $400.1315005 \mathrm{MHZ}$
\end{tabular}

PL2

PL12

DE

SFO
NUC
PL1

$-6.00 \mathrm{~dB}$

$18.00 \mathrm{~dB}$

6. 90 usec

100. 62з29з3 MHz

$13 \mathrm{C}$
$-6.00 \mathrm{~dB}$

F2 - Processing parameters

SI Processing parameters

$\begin{array}{lc}\text { SI } & 32768 \\ \text { SF } & 100.6127290 \\ \text { MHz }\end{array}$

$\begin{array}{lr}\text { WDW } & \text { EM } \\ \text { SSB } & 0\end{array}$

$\begin{array}{lc}\text { LB } & 1.00 \mathrm{HZ} \\ \text { GB } & 0\end{array}$

PC 1.40

10 NMA plot parameters

CX NMA plot parameters $220.036 \mathrm{ppm}$
$22138.42 \mathrm{~Hz}$ $-10.036 \mathrm{ppm}$ $-1009.73 \mathrm{~Hz}$

$11.50359 \mathrm{ppm} / \mathrm{m}$ $1157.40747 \mathrm{~Hz} / \mathrm{cm}$ 


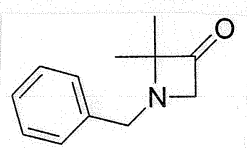

$6 a$
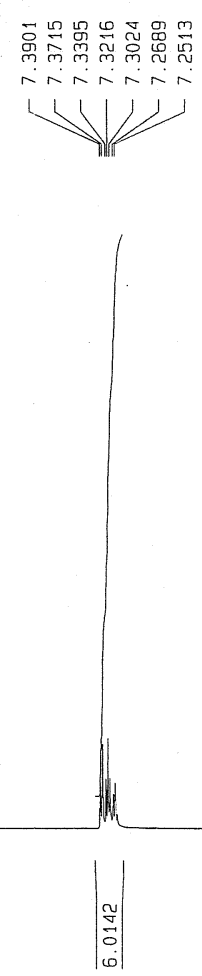

(1)
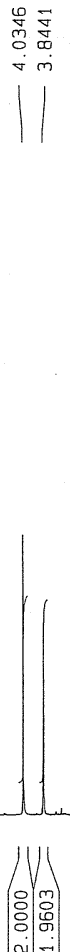

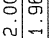

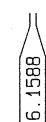

:
总

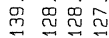

言

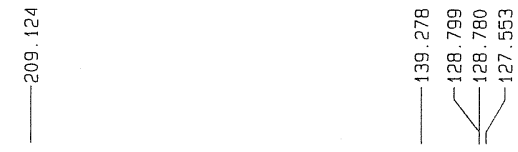

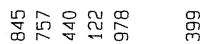

miño

$1 Y 1$

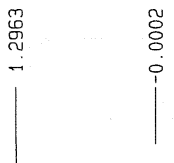

Current Data Parameters

NAME pnd-ij-140

PROCNO

F2 - Acquisition Parameters

$\begin{array}{lr}\text { Date } & 991122 \\ \text { Time } & 10.31\end{array}$

10.31
INSTAUM
Tr $\times 400$

PROBHD $5 \mathrm{~mm}$ Multinu

$\begin{array}{lr}\text { PULPAOG } & \text { Z930 } \\ \text { TD } & 32768\end{array}$

SOLVEN

$\begin{array}{ll}\text { OS } & \text { ? } \\ \text { SWH } & 4789.272 \mathrm{~Hz} \\ \text { FIDRES } & 0.146157 \mathrm{~Hz}\end{array}$

$0.146157 \mathrm{~Hz}$

3. $4210291 \mathrm{sec}$

104. 400 usec

4. 50 use

$1.00000000 \mathrm{sec}$

7.70 usec
4.50 usec

400. $1320007 \mathrm{MHz}$

$1 \mathrm{H}$
$-6.00 \mathrm{~dB}$

PL1

Fa - Processing parameters

SI 16384

$\begin{array}{lr}\text { SF } & 400.1300120 \mathrm{MHZ} \\ \text { WOW } & \text { EM }\end{array}$

SSB 0

$\begin{array}{cc}\text { LB } & 0.30 \mathrm{HZ} \\ \text { GB } & 0\end{array}$

10 NMR plot parameters

CX 20.00

$\begin{array}{lr}\text { CX } & 20.00 \mathrm{~cm} \\ \text { F1P } & 10.955 \mathrm{pom}\end{array}$

F1 $4383.25 \mathrm{~Hz}$

$\begin{array}{ll}F 2 P & -1.015 \mathrm{ppm} \\ \mathrm{F2} & -405.02 \mathrm{~Hz}\end{array}$

PPMCM $\quad-405.02 \mathrm{~Hz}$

HZCM $239.46359 \mathrm{~Hz} / \mathrm{cm}$

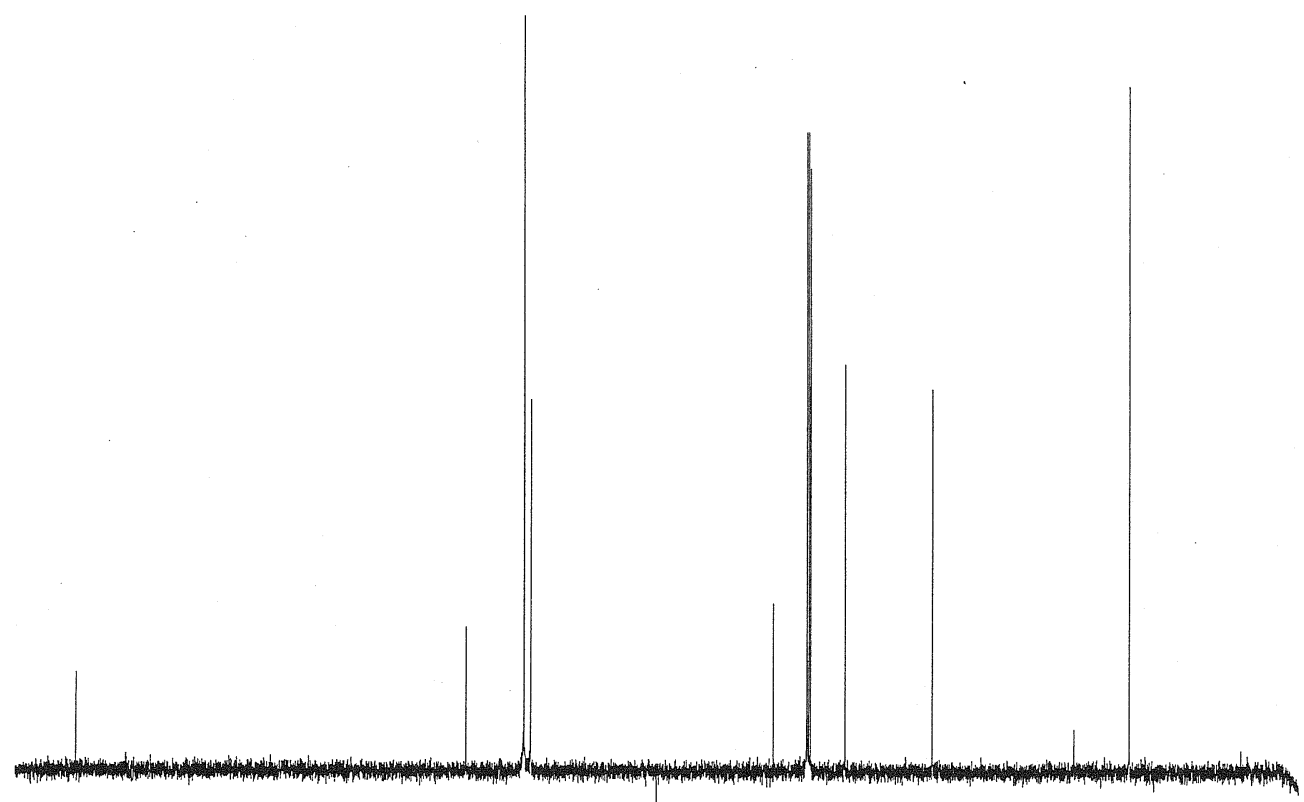

$$
\text { p }
$$

F2 - Acquisition Parameters

$\begin{array}{lr}\text { Date } & 991122 \\ \text { Time } & 10.37\end{array}$

$\begin{array}{lr}\text { Time } & 10.37 \\ \text { INSTRUM } & d r \times 400\end{array}$

PROBHD $5 \mathrm{~mm}$ Multinu

$\begin{array}{lr}\text { PULPPOG } & 290930 \\ \text { TO } & 65536\end{array}$

$\begin{array}{lr}\text { SOLVENT } & \text { COC13 } \\ \text { NS } & 509\end{array}$

$\begin{array}{ll}\text { DS } & 2 \\ \text { SWH } & 23148.148 \mathrm{~Hz}\end{array}$

$\begin{array}{lr}\text { SWH } & 23148.148 \mathrm{~Hz} \\ \text { FIDRES } & 0.353213 \mathrm{~Hz} \\ & 1.4156276 \mathrm{seC}\end{array}$

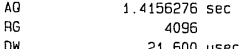

$\begin{array}{rr}\text { OW } & 21.600 \text { usec } \\ \text { DE } & 4.50 \text { usec }\end{array}$

TE $300.0 \mathrm{~K}$

$011 \quad 0.0300000 \mathrm{sec}$

$\begin{array}{lr}\text { d12 } & 0.0000200 \mathrm{sec} \\ \text { PL.13 } & 18.00 \mathrm{~dB}\end{array}$

$\begin{array}{ll}0.13 & 18.00 \mathrm{~dB} \\ 01 & 0.05000000 \mathrm{sec}\end{array}$

CPDPAG2 waltz16

$\begin{array}{ll}\text { PCPD2 } & 100.00 \text { usec }\end{array}$

NuC2

PL2

PL12

$P 1$
$P_{1}$
$D E$
$S F 01$

6.90 usec
4.50 usec

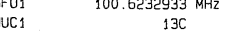

PL1 $-6.00 \mathrm{~dB}$

$\begin{array}{lc}\text { F2 } & \text { - Processing parameters } \\ \text { SI } & 32768 \\ \text { SF. } & 100.6127290 \mathrm{MHz}\end{array}$

SF. $\quad 100.6127290$

$\begin{array}{lc}\text { SSB } & \text { EM } \\ \text { LB } & 0 \\ \text { GB } & 1.00 \mathrm{~Hz} \\ & 0\end{array}$

10 NMP plot paraneters

CX $\quad 20.00 \mathrm{~cm}$

$\begin{array}{lr}220.036 & \mathrm{ppm} \\ & 22138.41 \mathrm{~Hz}\end{array}$

F2P $\quad-10.036 \mathrm{ppm}$

F2 $\quad-1009.74 \mathrm{~Hz}$

$\begin{array}{lr}\text { PPMCM } & 11.50359 \mathrm{ppm} / \mathrm{cm} \\ \text { HZCM } & 1157.40747 \mathrm{~Hz} / \mathrm{Cm}\end{array}$ 


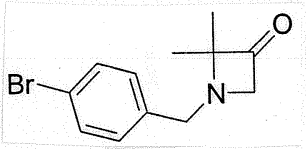

6b

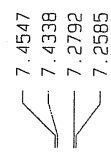

il
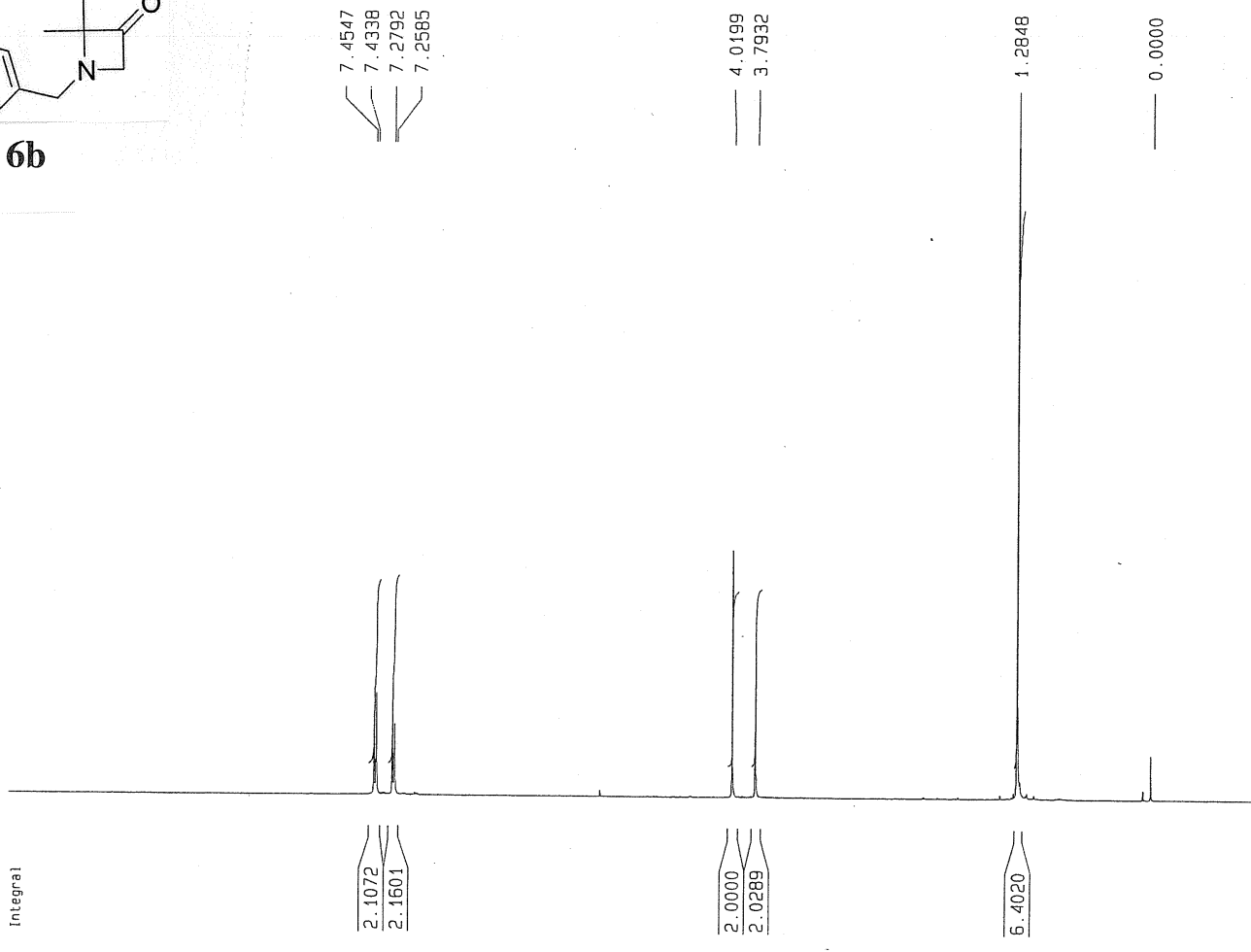

ppm
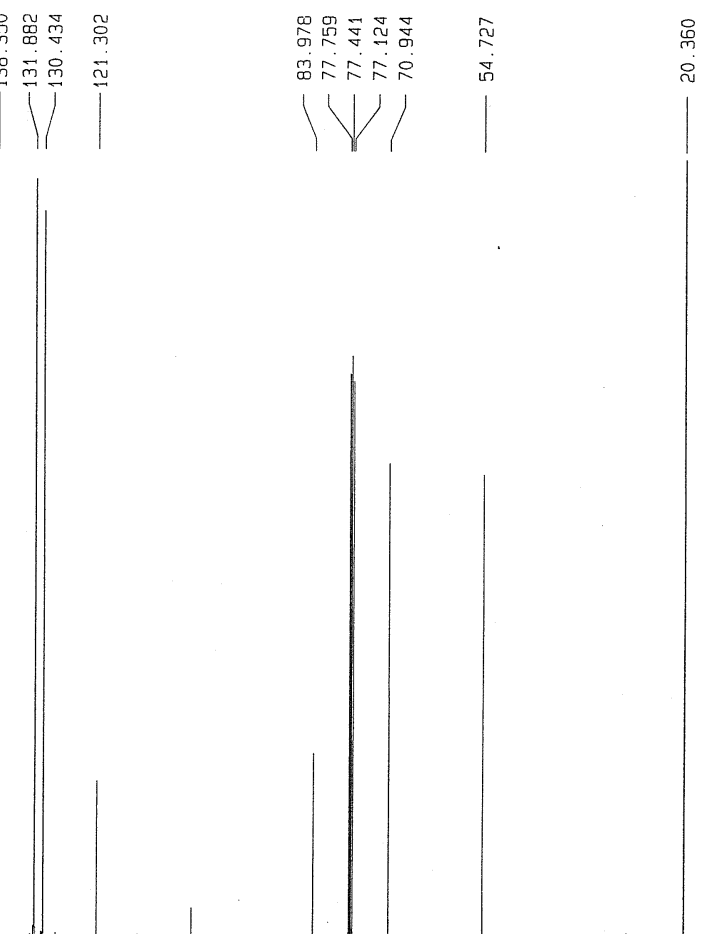

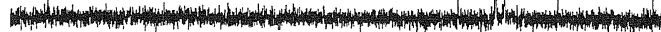

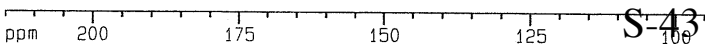

150

12

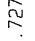

in
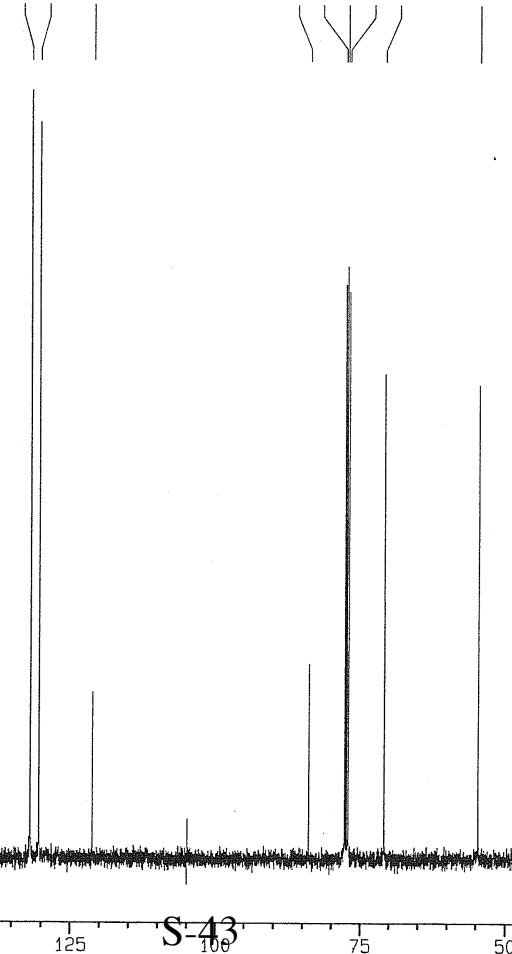


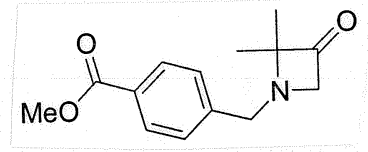

$6 c$

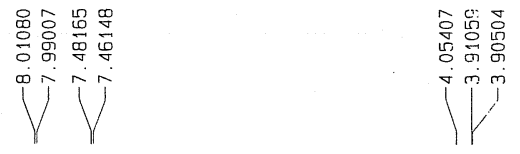

음 땅

|
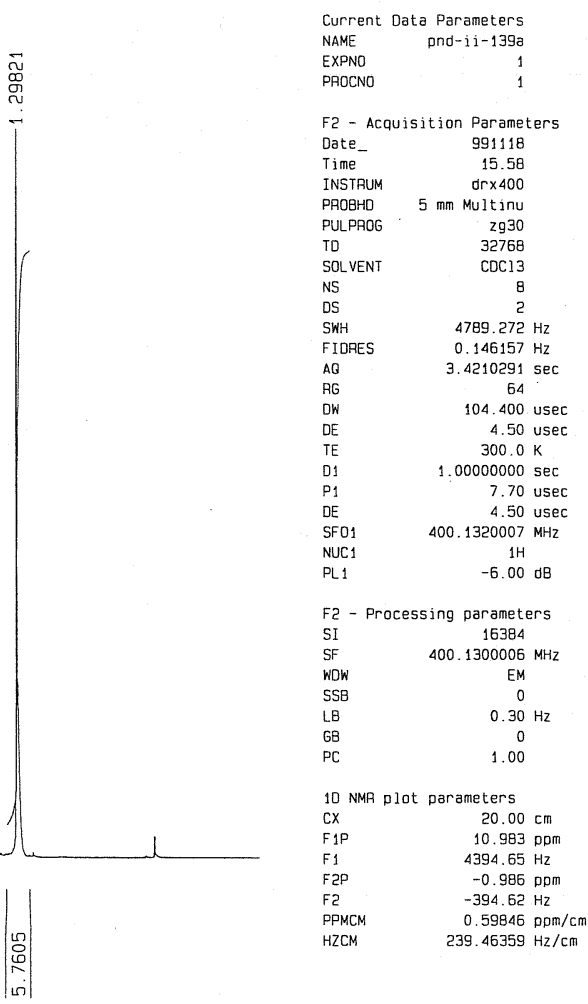

ppm

10
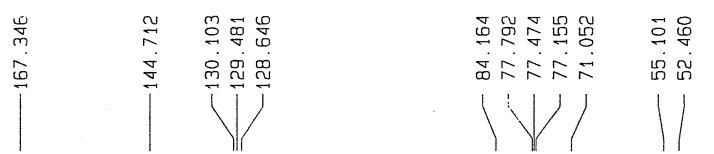

8

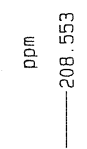

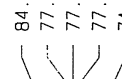

ก

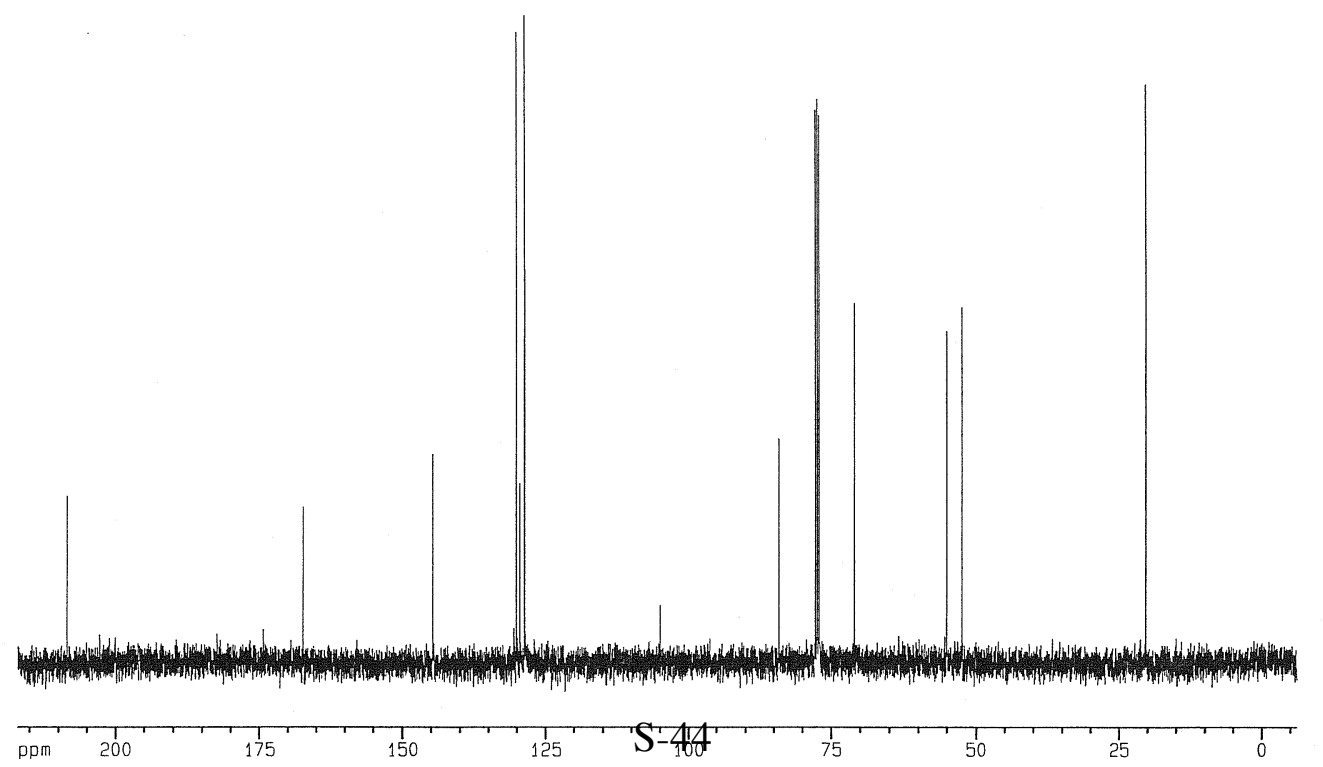




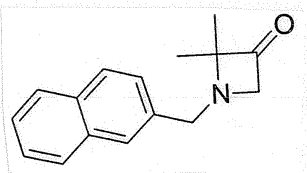

$6 d$

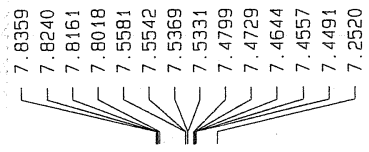

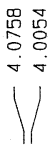
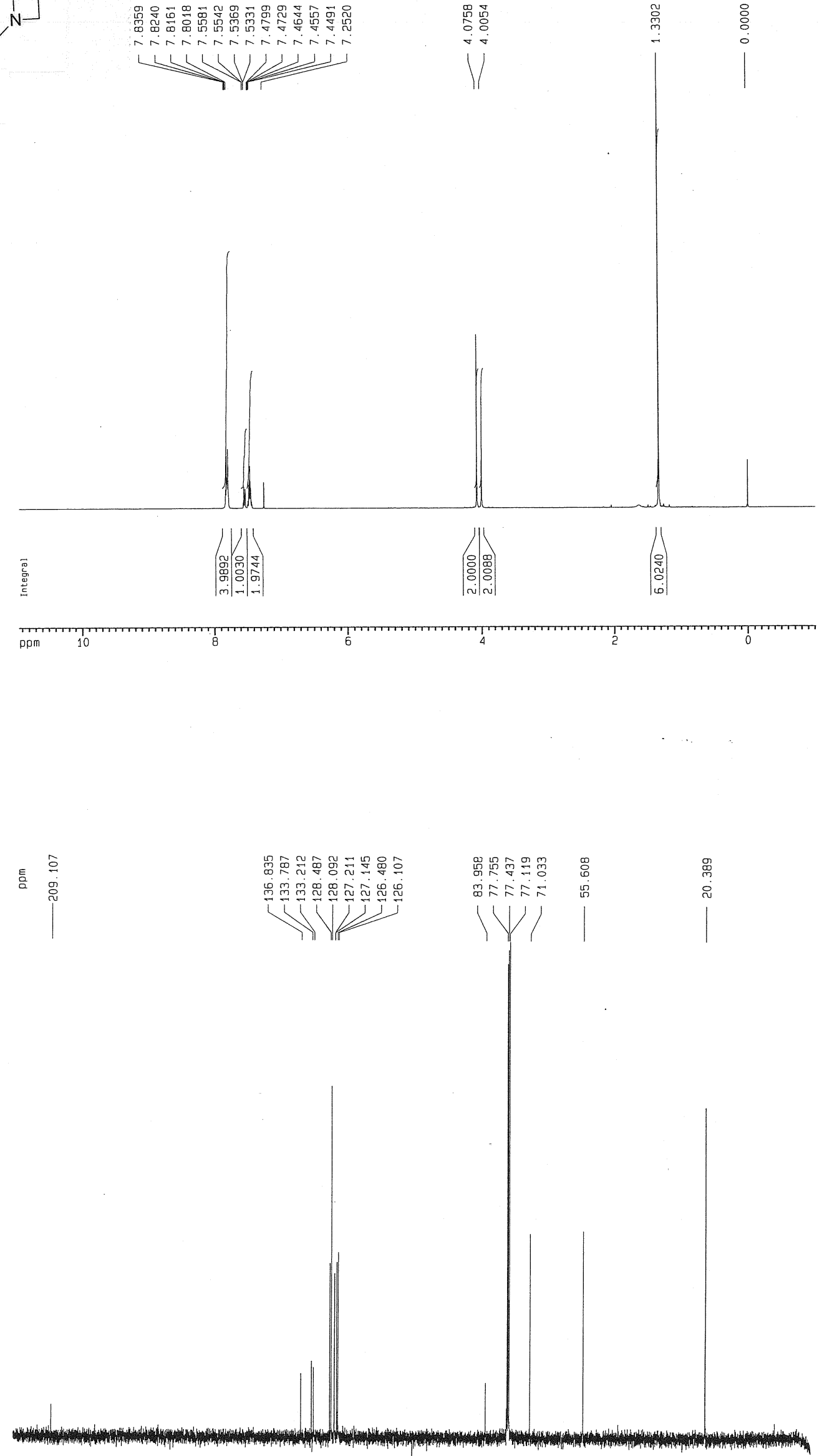

pp

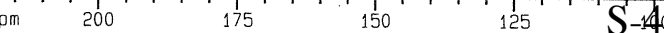

Current Data Parameters

NAME $\quad$ pnd-1i-96a

PROCNO

F2 - Acquisition Parameters

Date_ $\quad 991112$

NSTRUM 10.33

PAOBHD $5 \mathrm{~mm}$ Multinu

PULPAOG 2930

$\begin{array}{ll}\text { TD } & 32768 \\ \text { SOLVENT } & \text { CDC13 }\end{array}$

NS

DS 2

$\begin{array}{ll}\text { SHH } & 4789.272 \mathrm{~Hz} \\ \text { FIDRES } & 0.146157 \mathrm{~Hz}\end{array}$

AQ $\quad 3.4210291 \mathrm{sec}$

228.1
104.400 use

104.400 use

4.50 usec
$300.0 \mathrm{~K}$

1. $00000000 \mathrm{sec}$

7.70 usec
4.50 usec

400. $1320007 \mathrm{MHz}$

$-6.00 \mathrm{~dB}$

PL1

F2 - Processing parameters

SI 16384

$\begin{array}{lc}\text { SF } & 400.1300129 \mathrm{MHz} \\ \text { WDW } & \text { EM }\end{array}$

$\begin{array}{ll}\text { SSB } & 0 \\ \text { LB } & 0.30 \mathrm{~Hz}\end{array}$

$\begin{array}{lr}\mathrm{PB} & 0 \\ \mathrm{PC} & 1.00\end{array}$

10 NMA plot parameters

CX $20.00 \mathrm{~cm}$ $\begin{array}{lr}\text { F1P } & 10.952 \mathrm{ppm} \\ \text { F1 } & 4382.37 \mathrm{~Hz}\end{array}$ $10.9 .37 \mathrm{~Hz}$
$-1.017 \mathrm{pon}$ F2 $\quad-406.90 \mathrm{~Hz}$ $\mathrm{HZCM}$
$239.46359 \mathrm{~Hz} / \mathrm{cm}$

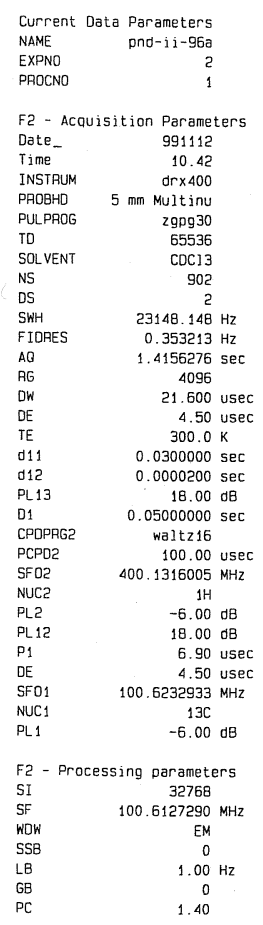

10 NMA plot parameters

10 NMA plot parameters
CX $20.00 \mathrm{~cm}$ $\begin{array}{ll}\text { F1P } & 220.036 \mathrm{ppm} \\ \mathrm{Fi} & 23138.42 \mathrm{~Hz}\end{array}$ $\begin{array}{lr}\text { F1 } & 22138.42 \mathrm{~Hz} \\ \text { F2P } & -10.036 \mathrm{pD}\end{array}$ F2 $\quad-1009.73 \mathrm{~Hz}$ PPMCM $\quad 11.50359 \mathrm{ppm} / \mathrm{Cm}$ 

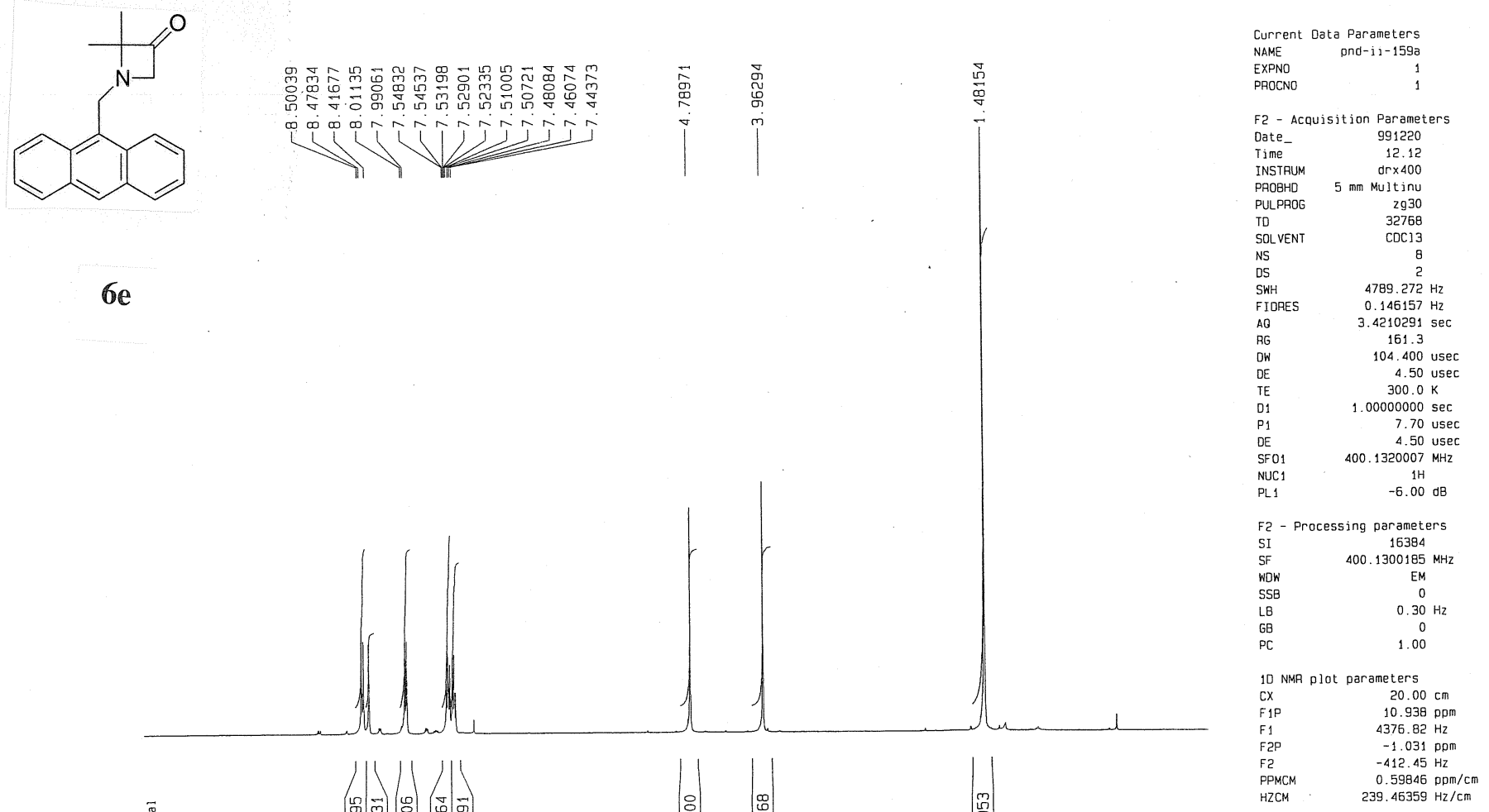

$6 e$
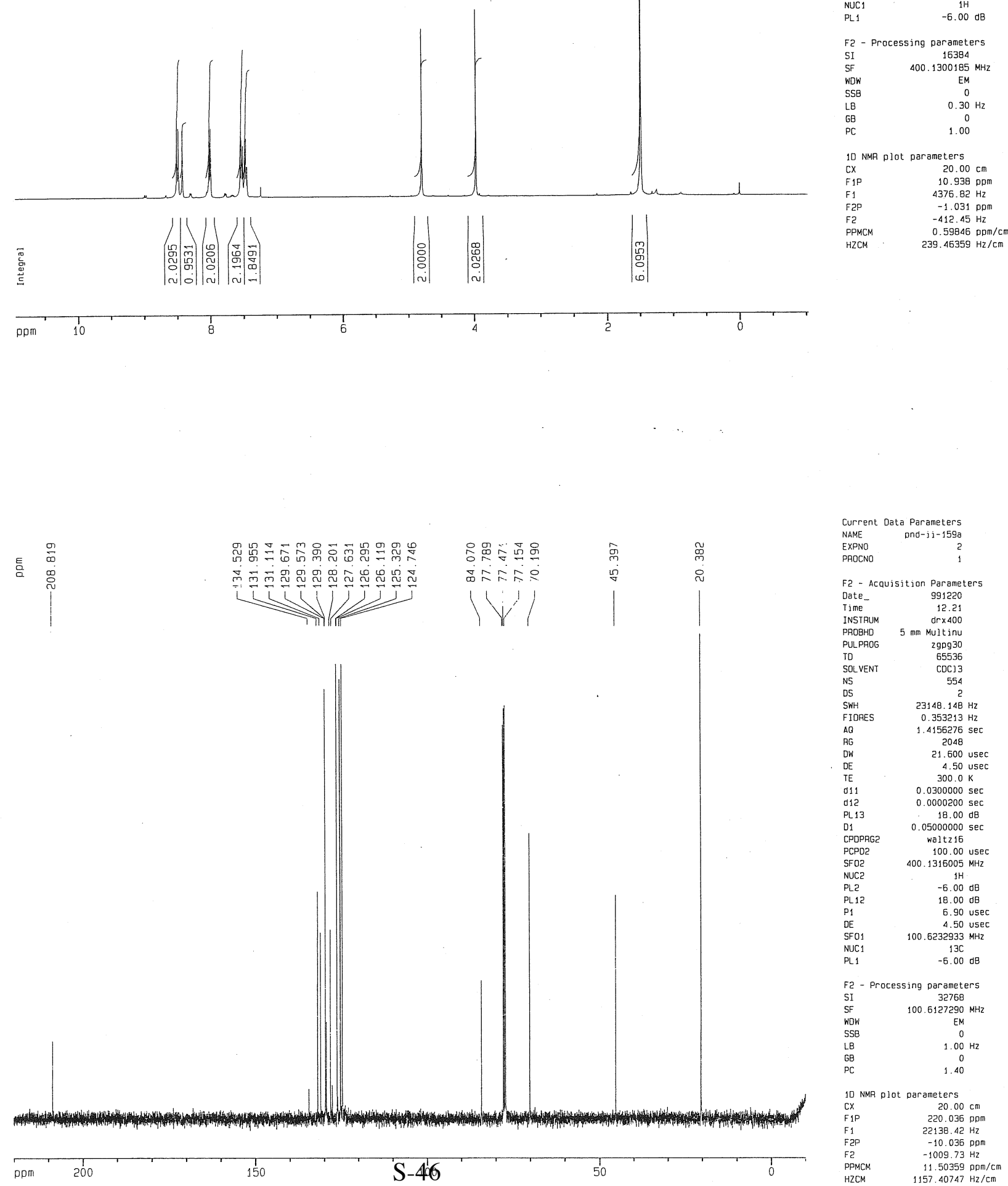


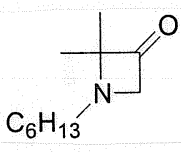

$6 f$
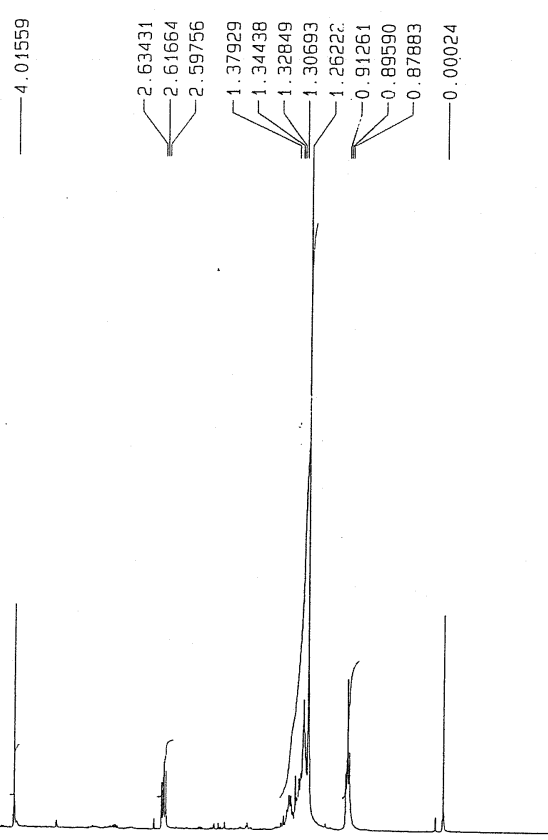

$\left|\begin{array}{|l}\mid \\ 0 \\ 0 \\ ن\end{array}\right|$

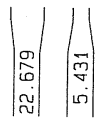

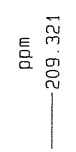

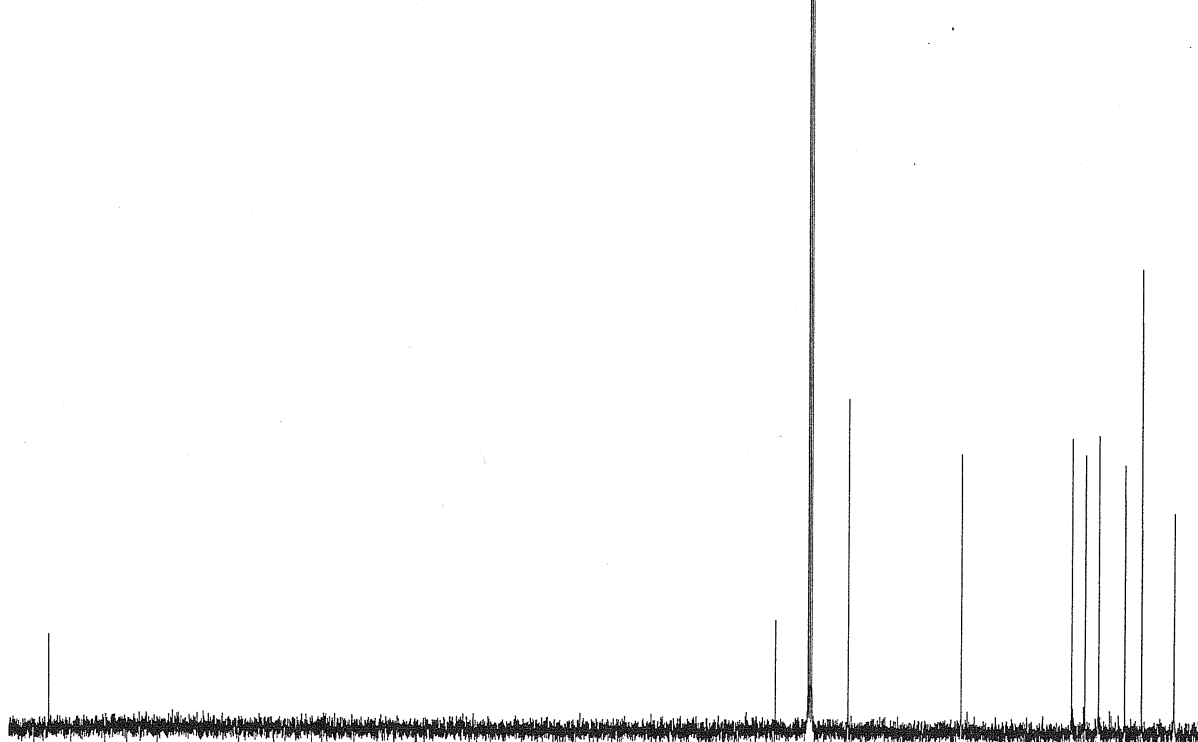

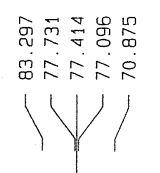

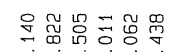

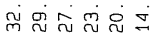

\1111

$\overline{p p m}$ 
01

$9 a$

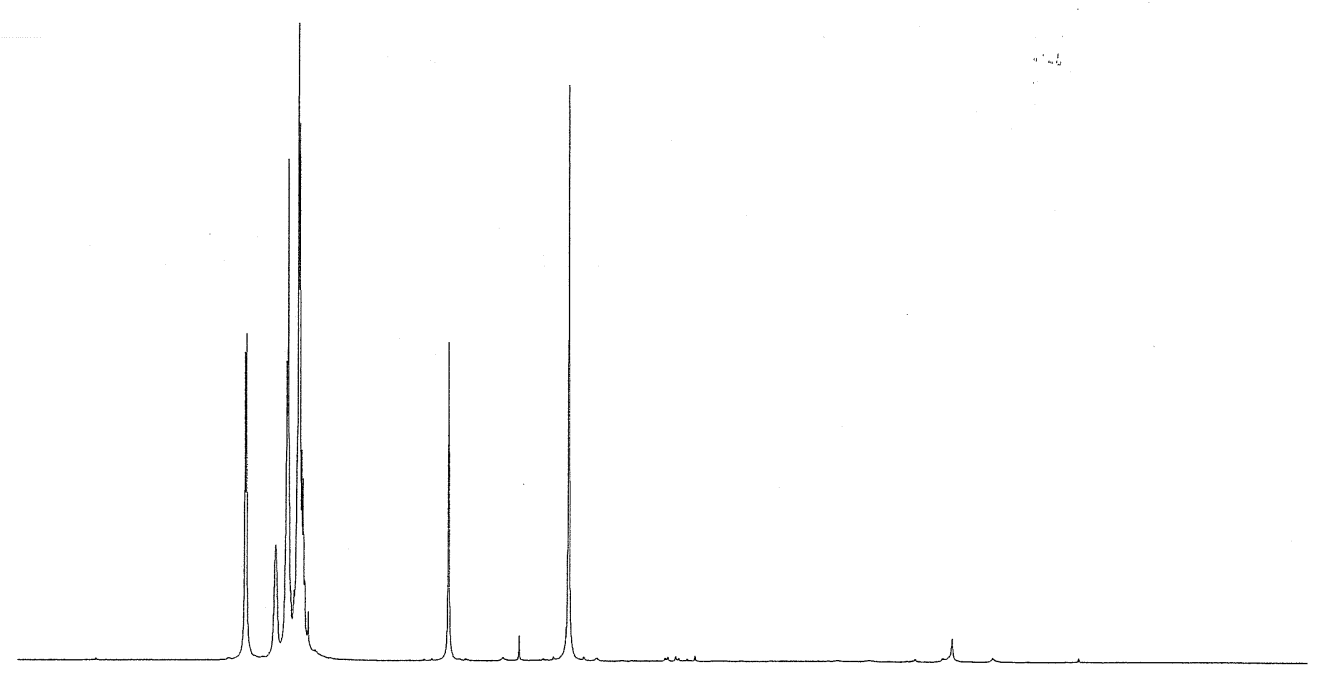

ppm

6

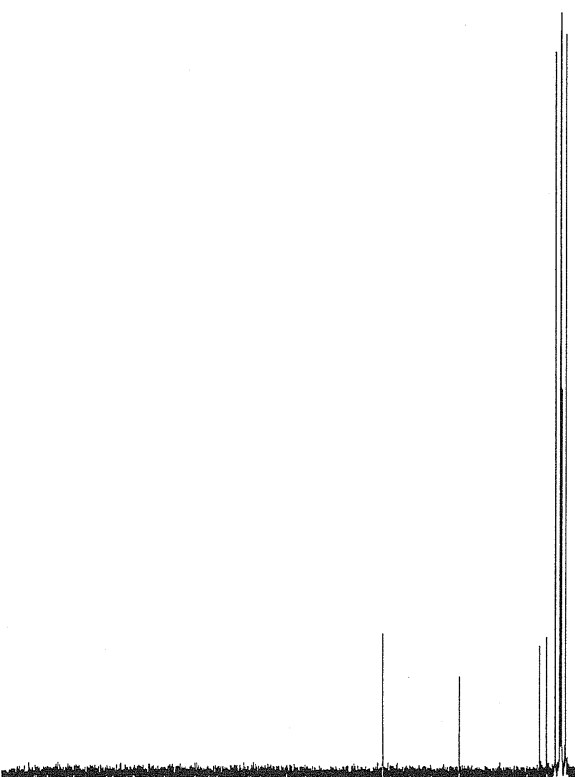

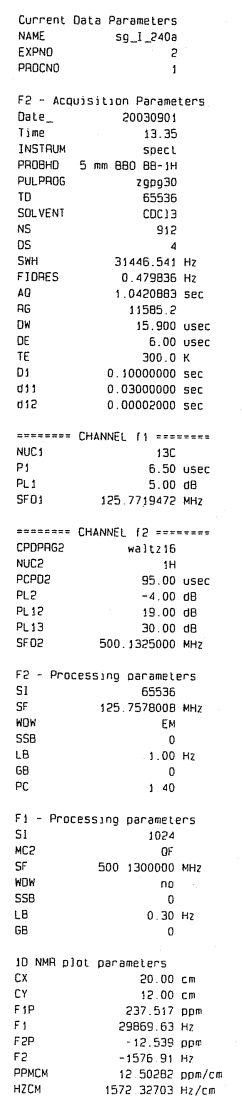


21

$9 b$
Current Data Parameters
naMe

EXPNO

F2 - Acquisition Parameters

Date_ 20030915

$\begin{array}{ll}\text { Time } & 11.33 \\ \text { INSTRUM } & 50 \mathrm{Cl}\end{array}$

PDect
PAOBHD $5 \mathrm{~mm}$ BBO BQ-1H

$\begin{array}{lr}\text { PULPROG } & 2930 \\ \text { TO } & 32768 \\ \text { SOLVNT } & \text { COC13 }\end{array}$

NS

FIORES

AQ

$D W$
$D E$
TE

D1

$z=z=z=2$ CHANNEL $f 1=z=z=n$
NUC1

17.70 usec

$\begin{array}{lr}\text { PL1 } & -4,00 \mathrm{~dB} \\ \text { SF } 01 & 500.1320005 \mathrm{MHZ}\end{array}$

F2 - Processing parameters
SI 32768

$\begin{array}{lc}\text { SF } & 500.1300000 \mathrm{MH} \\ \text { WOW } & \text { EM }\end{array}$

$\begin{array}{lc}\text { WOW } & \text { EM } \\ \text { SSB } & 0 \\ \text { LB } & 0.30 \mathrm{HZ}\end{array}$

$\begin{array}{lr}\mathrm{GB} & 0 \\ \mathrm{PC} & 1.00\end{array}$

F1 - Processing parameters

II Processing parameters

$\begin{array}{lc}\text { MC2 } & 1024 \\ \text { SF } & \text { GF } \\ \text { SF } & 500.1300000 \\ \text { MH-1 }\end{array}$

SSB

QSINE
0
$0.30 \mathrm{HZ}$

0 NMA plot parameters

ex $20.00 \mathrm{~cm}$

$\begin{array}{lc}\text { CY } & 10.00 \mathrm{~cm} \\ \text { F IP } & 10.000 \mathrm{ppm}\end{array}$

$\begin{array}{lr}F_{1} & 5005.33 \mathrm{~Hz} \\ F_{22} & -2.008 \mathrm{ppm}\end{array}$

PPMCM $\quad-1004.29 \mathrm{~Hz}$

$\begin{array}{ll}\text { HZCM } & 0.60081 \mathrm{ppm} / \mathrm{cm} \\ & 300.48077 \mathrm{~Hz} / \mathrm{cm}\end{array}$

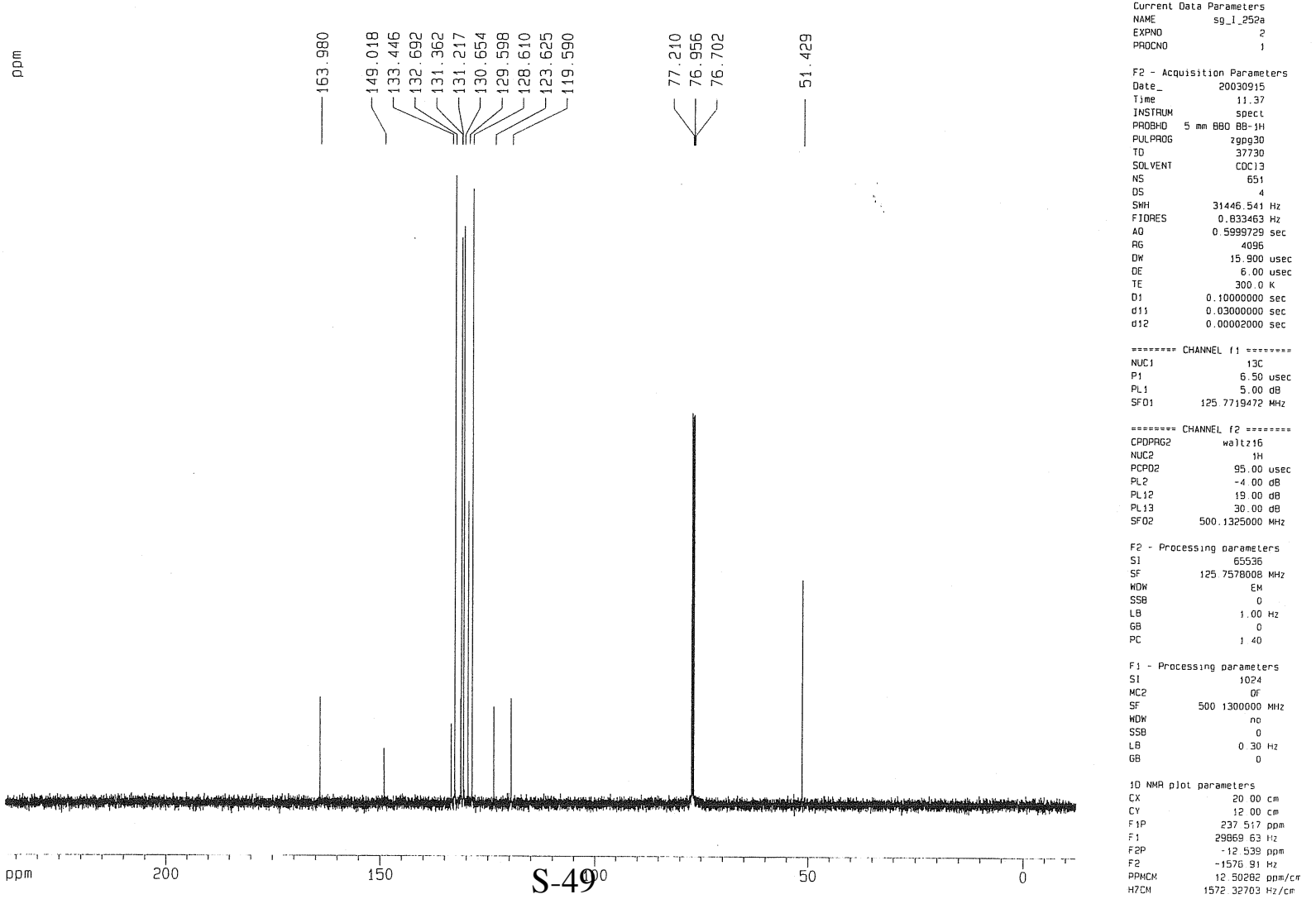




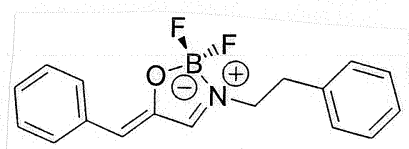

$9 c$

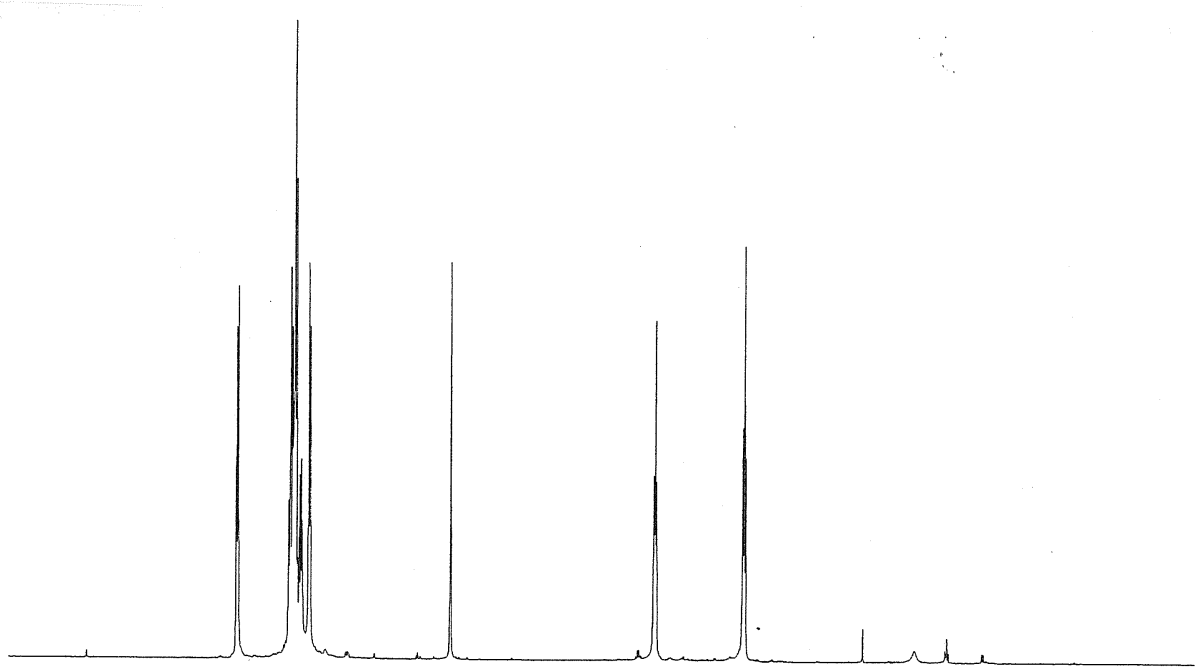

ppm

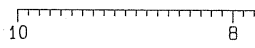

公

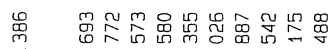

苛

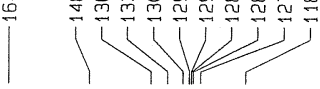

筞䍘品

مம

V

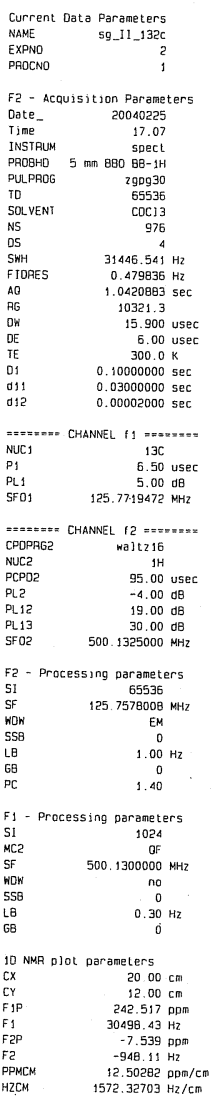




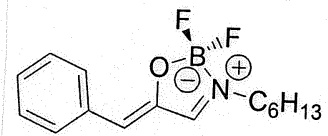

9d

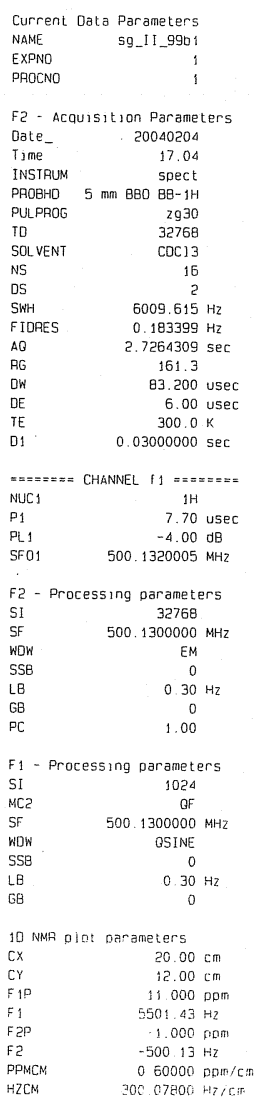

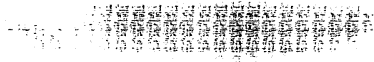

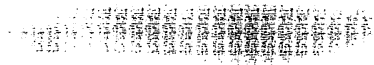

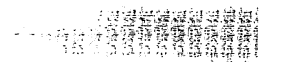

言

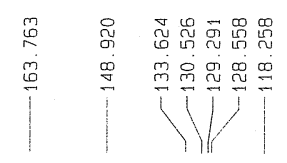

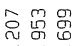

둥용

Y

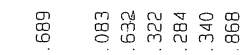

๓ூ丁

Hil

\section{4}

ppin

${ }^{\prime}{ }^{1} 0^{\circ}$

${ }^{1}{ }^{1} 0^{\prime}$

S-51 100
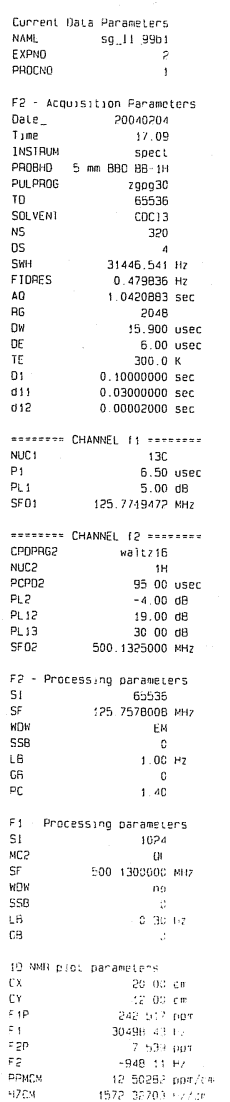


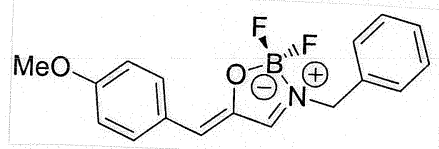

$9 \mathrm{e}$
Current Data Parameters

EXPNO

F2 - Acquisition Parameters

PULPROG

2930
TO

SWH

FIDRES

AQ

RG
DW
DE
TE
$D 1$

$==$

$==$
NUC

$\mathrm{P} 1$
$\mathrm{PL}$
$\mathrm{S}$

F2 - Processing parameters
SI 32768

$\begin{array}{lc}\mathrm{SF} & 500.1300000 \mathrm{MHZ} \\ \text { WOW } & \mathrm{EM}\end{array}$

WOW

LB

1.00

F1 - Processing parameters
SI 1024

$\begin{array}{lc}\text { MC2 } & \text { OF } \\ \text { SF } & 500.1300000 \mathrm{MHz}\end{array}$

WOW
SSB
LB
GB

QSINE
0
$0.30 \mathrm{~Hz}$

10 NMP plot parameters

CX $20.00 \mathrm{~cm}$

$10.00 \mathrm{~cm}$

$10.000 \mathrm{ppm}$

$-2.008 \mathrm{ppm}$
$-204.29 \mathrm{~Hz}$

$-1004.29 \mathrm{~Hz}$

$0.60081 \mathrm{ppm} / \mathrm{cm}$
$000.48077 \mathrm{~Hz} / \mathrm{cm}$ ppm

${ }_{6}^{17} 1$

2

这离品

م

$V$
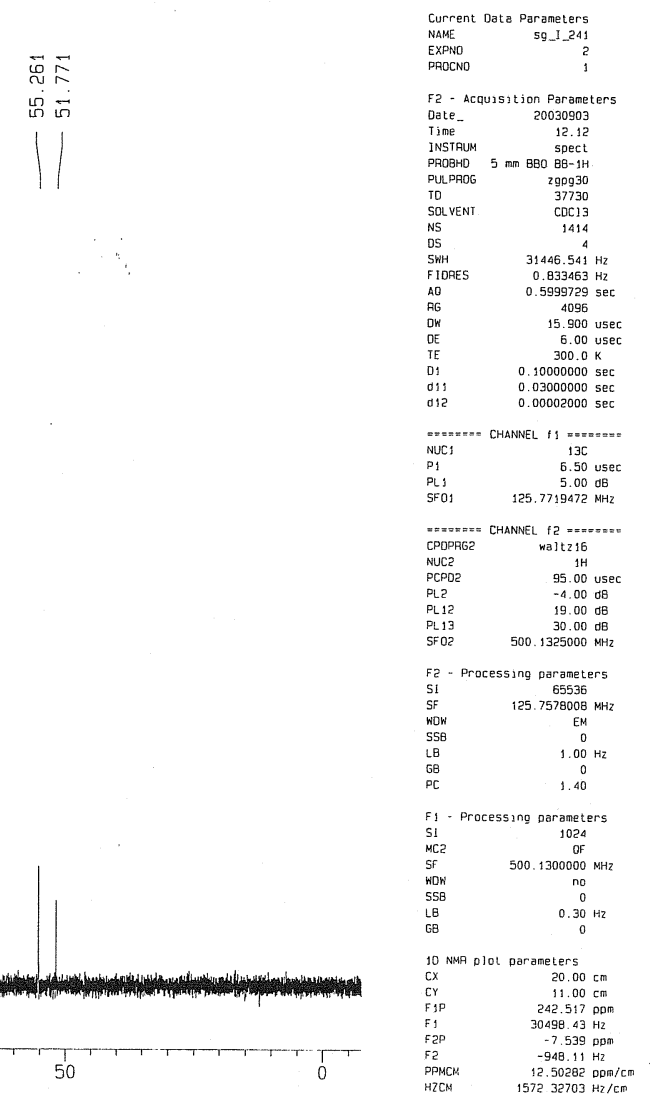

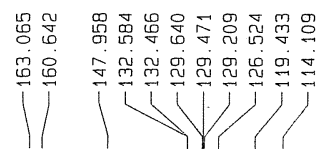

||

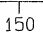

$S-52^{10}$
50

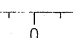

200

150

150 


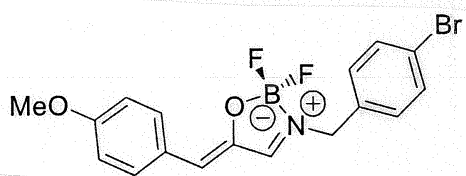

9f

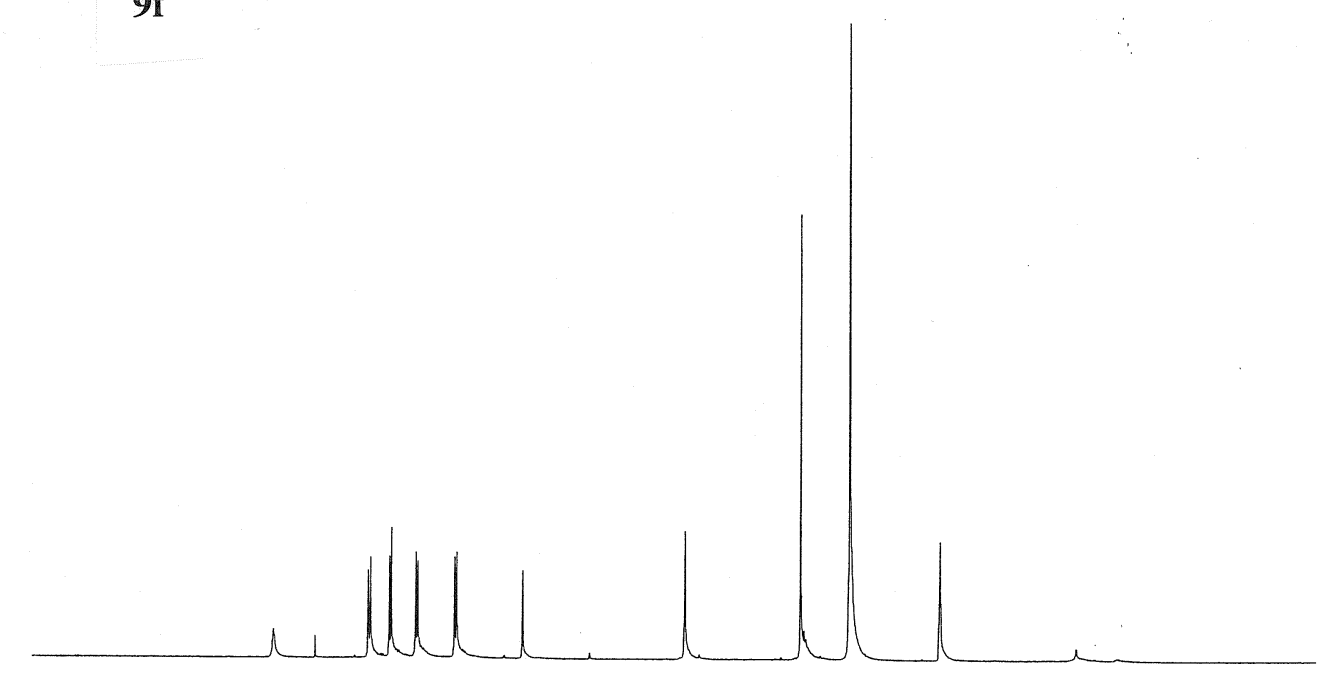

Current Data Parameters
NAME

F2 - Acquisition Parameters

Date_ $\quad 20030908$

Time

5 drx400

PROBHD 5 m

TD

NS

16
2

$\begin{array}{ll}4789.272 \mathrm{~Hz} \\ \text { FIDRES } & 0.146157 \mathrm{~Hz}\end{array}$

AQ $\quad 3.4210291 \mathrm{sec}$

$\begin{array}{lr}\text { RG } & 100 \\ \text { DW } & 104.400 \text { use }\end{array}$

DE $\quad 4.50$ use

$300.0 \mathrm{~K}$

D1 $\quad 1.00000000 \mathrm{sec}$

$=======$ CHANNEL f1 $==z====$
NUC1 $1 \mathrm{H}$

$\begin{array}{lr}\text { NUC1 } & 1 \mathrm{H} \\ \text { P1 } & 7.70 \text { usec } \\ \text { PL1 } & -6.00 \mathrm{~dB} \\ \text { SF01 } & 400.1320007 \mathrm{MHz}\end{array}$

F2 - Processing parameters

SI $\quad 16384$

WF $\quad 400.1300000$

SSB 0.30

$\begin{array}{lc}\text { LB } & 0.30 \mathrm{~Hz} \\ \text { GB } & 0 \\ \text { PC } & 1.00\end{array}$

ID NMR plot parameters

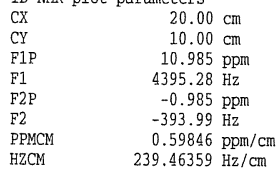

ppm 10

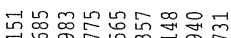

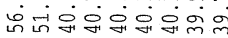

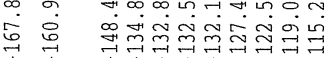

镸

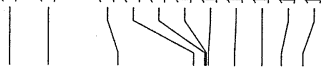

$\begin{array}{ll}\text { Current Data Parameters } \\ \text { NAME } & \text { Sg_I_245d }\end{array}$

EXPN

F2 - Acquisition Parameters

Date 20030908

INSTRUM drx400

PROBHD 5 mm Multinuc1

\begin{tabular}{lr} 
PULPROG & 2gpg30 \\
TD & 32404 \\
\hline
\end{tabular}

SOLVENT

$\begin{array}{lr}\text { NS } & 1362 \\ \text { DS } & 2 \\ \text { SWH } & 23148.148\end{array}$

FIDRES $\quad 0.714361 \mathrm{~Hz}$

$\begin{array}{lc}\text { AQ } & 0.6999764 \mathrm{sec} \\ \text { RG } & 4096\end{array}$

21.600 usec

4.50 usec
$300.0 \mathrm{~K}$

$.05000000 \mathrm{sec}$

0.00002000 sec

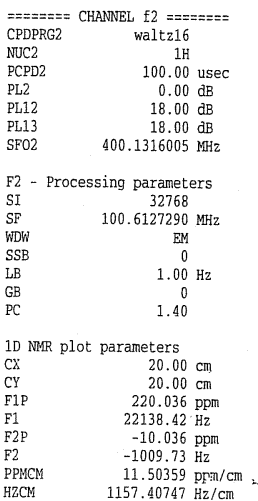



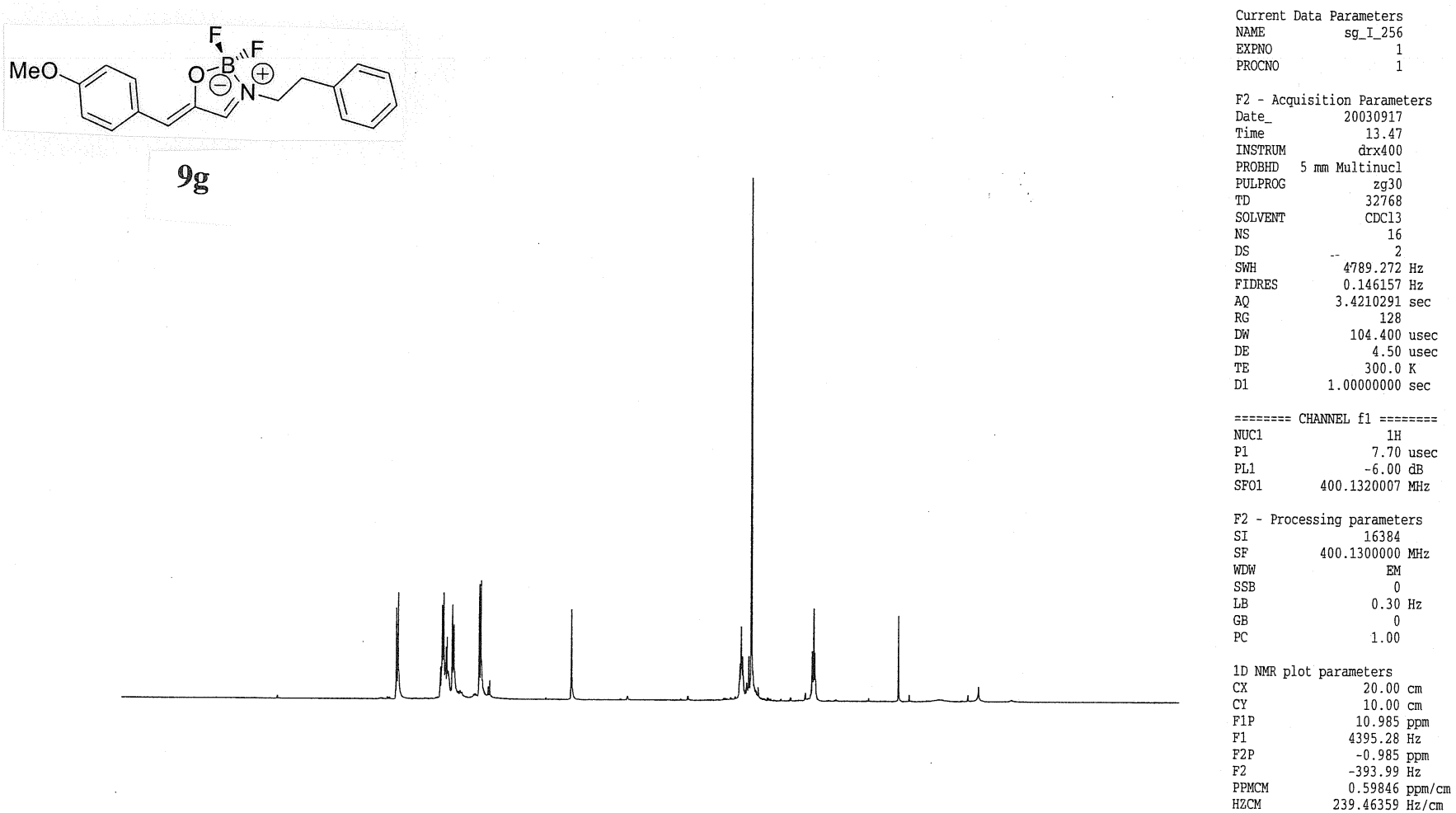

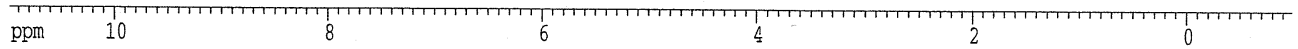

言

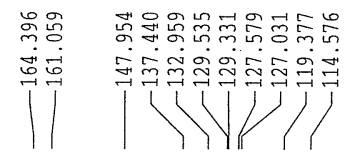

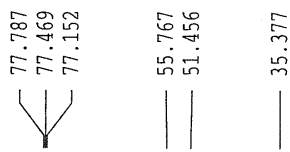
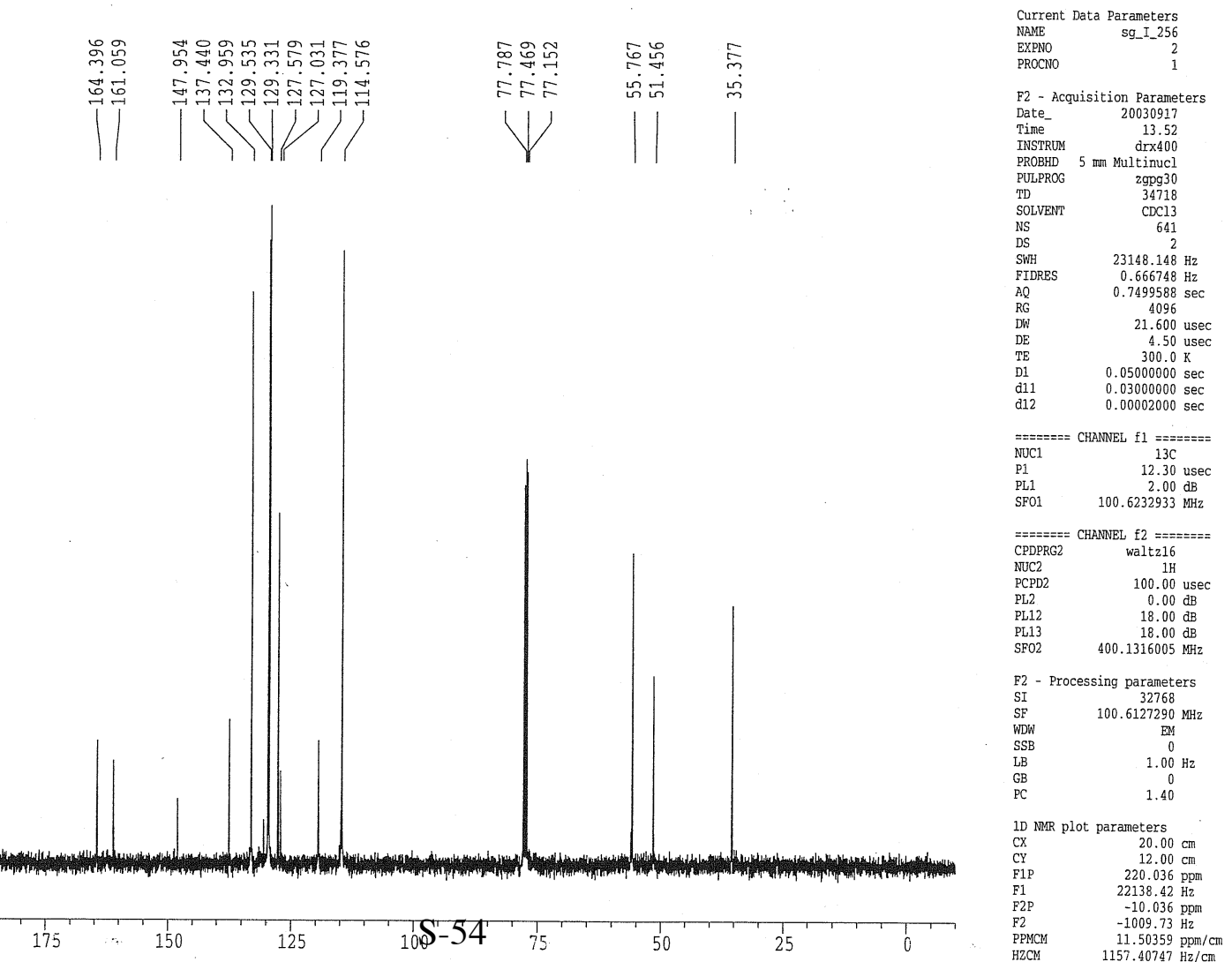

${ }_{100}^{1}-54$ 

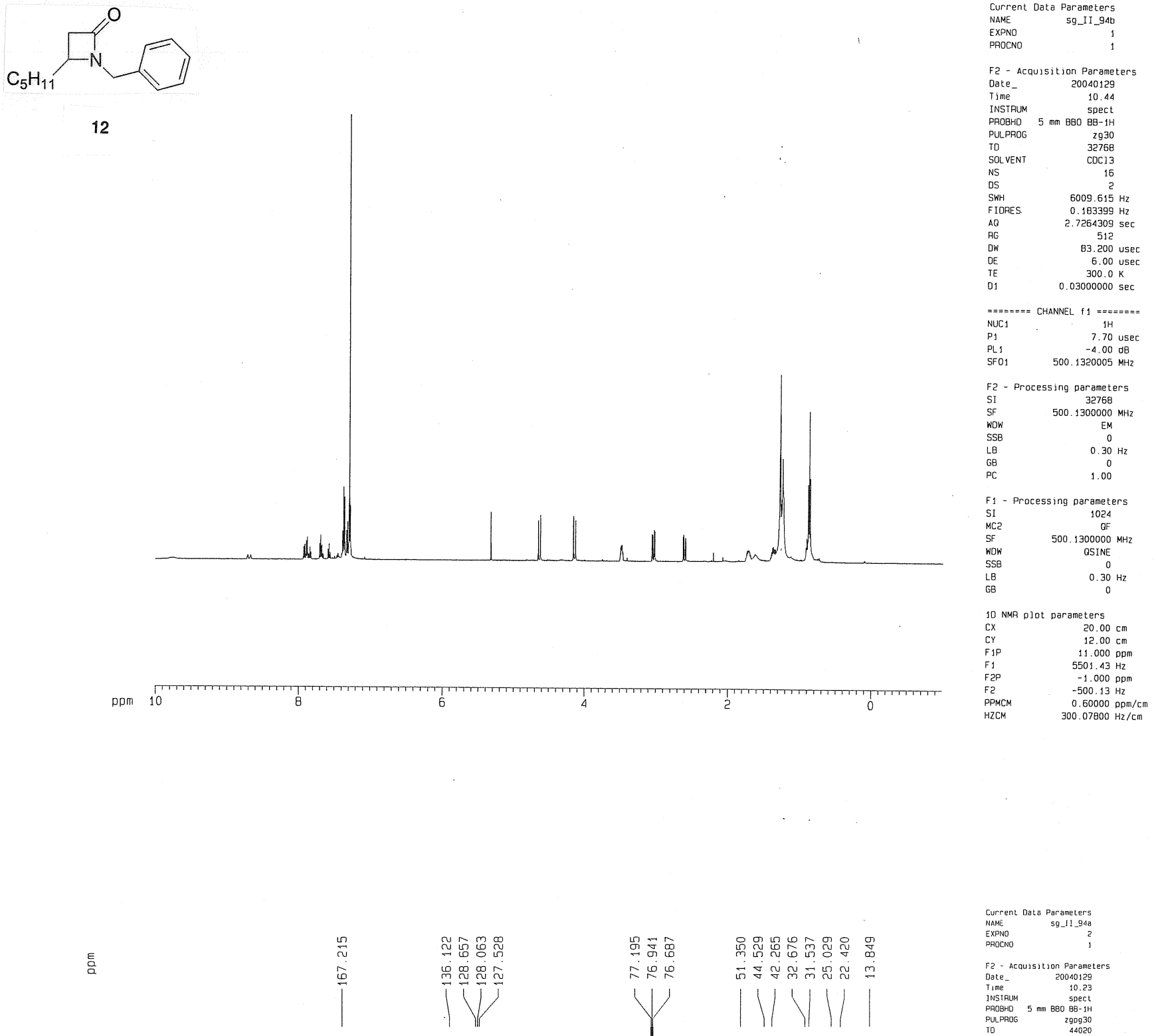

苟学总

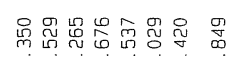

र्ट்

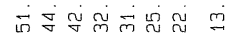

흠

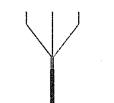

11111
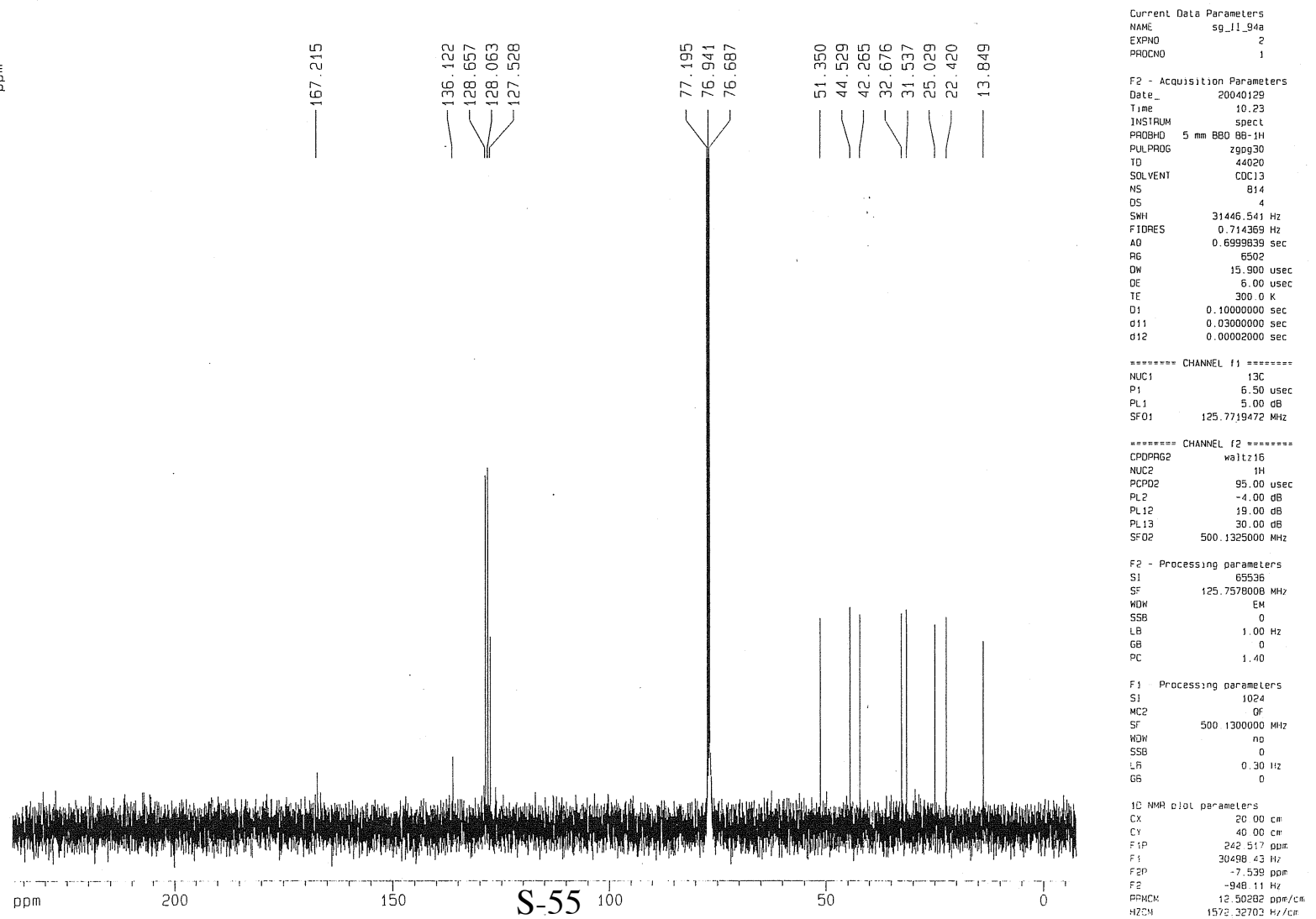
$\mathrm{C}_{5} \mathrm{H}_{11}$

13

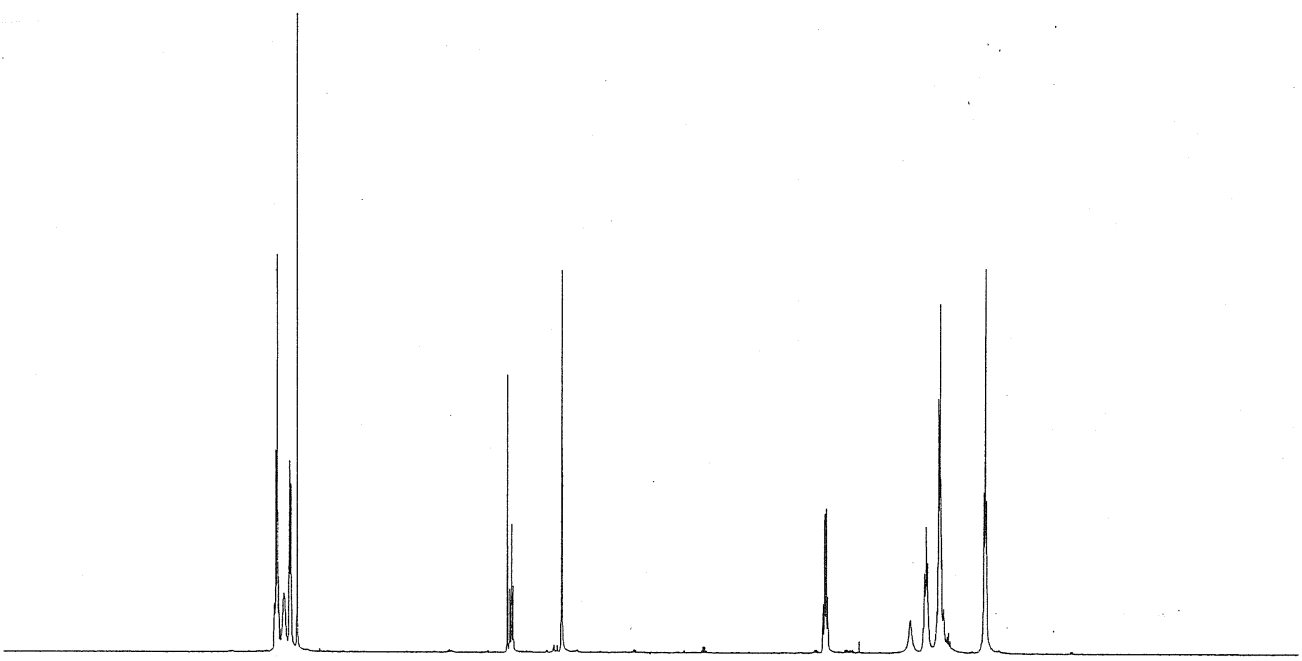

ppm

8

言
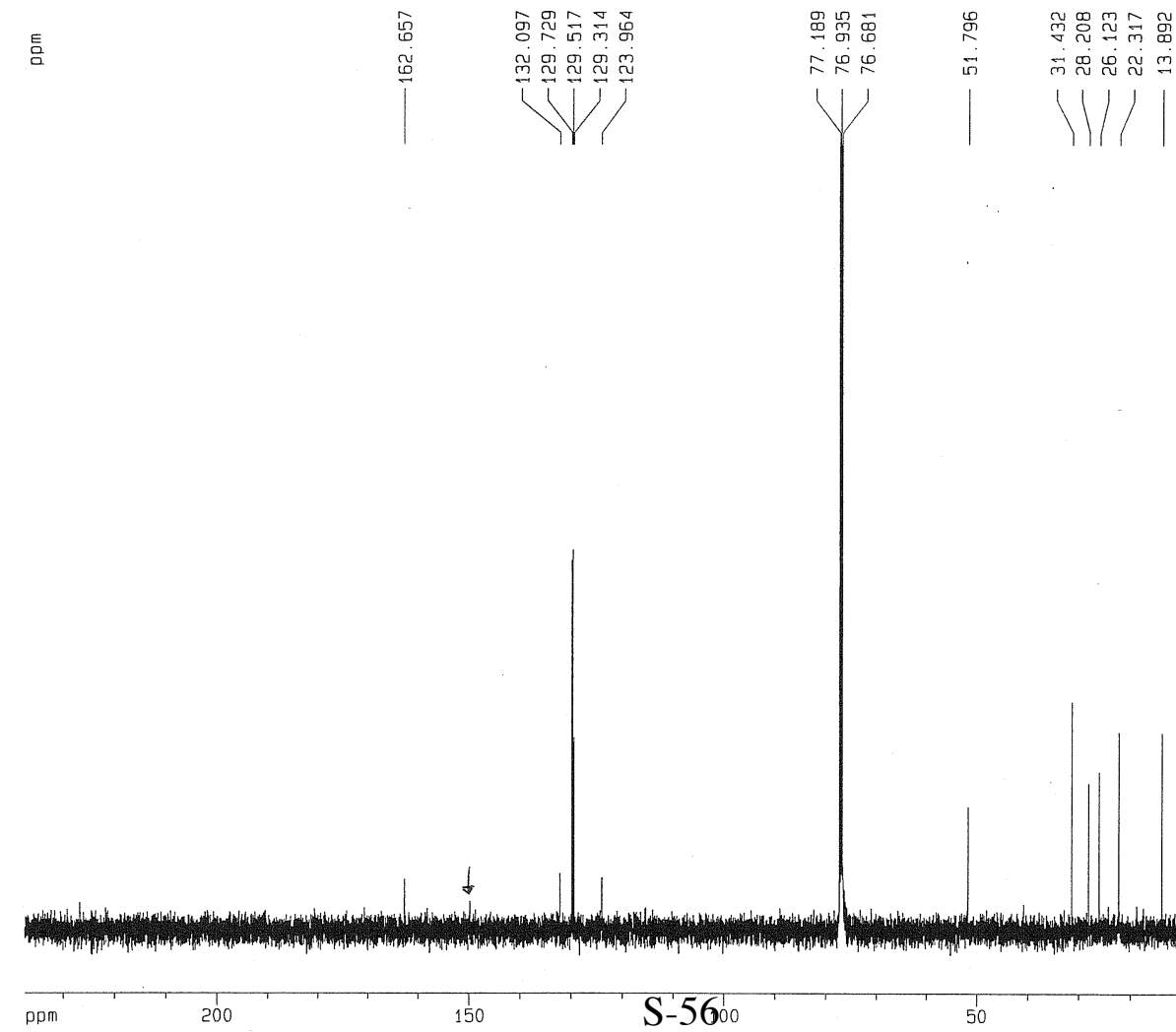

$\mathrm{ppm}$

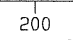

150

S-5600

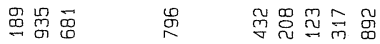

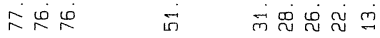
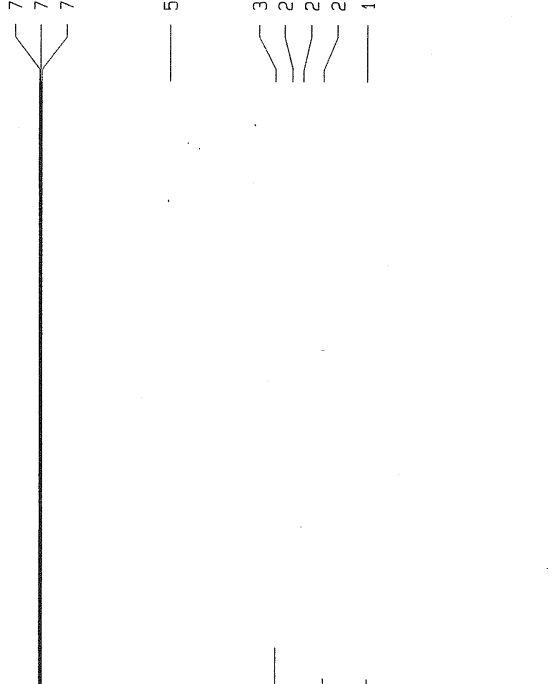

$\begin{array}{lr}\text { Current } & \text { Data Parameters } \\ \text { NAME } & \text { Sg_II_85a } \\ \text { EXPNO } & 1 \\ \text { PROCNO } & 1\end{array}$

F2 - Acquisition Parameters

Date $\quad 20040126$

Time

PAOBHO $5 \mathrm{~mm}$ BBO BPect

TO

NS

DS

FIOP

$A B$
$\mathrm{AG}$

$\mathrm{RG}$
$\mathrm{DW}$
$\mathrm{DE}$
$\mathrm{TE}$

TE

$== \pm= \pm==$ CHANNEL $f 1=== \pm= \pm==$

$\begin{array}{lr}\text { NUC1 } & 1 \mathrm{H} \\ \mathrm{P}_{1} & 7.70 \mathrm{usec}\end{array}$

$\begin{array}{lr}\text { PL1 } & -4.00 \mathrm{~dB} \\ \text { SF01 } & 500.1320005 \mathrm{MHz}\end{array}$

F2 - Processing parameters

SI 32768

$\begin{array}{lc}\text { SF } & 500.1300000 \\ \text { WOW } & \text { EM } \\ \text { SSB } & 0\end{array}$

$\begin{array}{ll}\text { SSB } & 0.30 \mathrm{~Hz} \\ \text { LB } & 0 \\ \text { GB } & 1.00 \\ \text { PC } & \end{array}$

F1 - Processing parameters

$\begin{array}{ll}\text { SI } & 1024 \\ \text { MC2 } & \text { DF }\end{array}$

$\begin{array}{cc}\text { MC2 } & \text { OF } \\ \text { SF } & 500.1300000 \\ \text { MHW } & \text { OSINE }\end{array}$

$\begin{array}{cc}\text { SSB } & 0 \\ \text { LB } & 0.30 \mathrm{~Hz} \\ \text { GB } & 0\end{array}$

I0 NMP plot paraneters

10 NMA plot parameters
$\mathrm{CX}$
$\mathrm{CY}$
$20.00 \mathrm{~cm}$

$\begin{array}{lr}\text { F1P } & 10.00 \mathrm{~cm} \\ F 1 & 10.000 \mathrm{ppm} \\ F_{2 p} & 5005.33 \mathrm{~Hz} \\ F & -2.000 \mathrm{pom}\end{array}$

$\begin{array}{lr}\text { F2P } & -2.000 \mathrm{ppm} \\ \mathrm{F} & -1004.29 \mathrm{~Hz}\end{array}$

PPMCM $\quad 0.60081 \mathrm{ppm} / \mathrm{cm}$

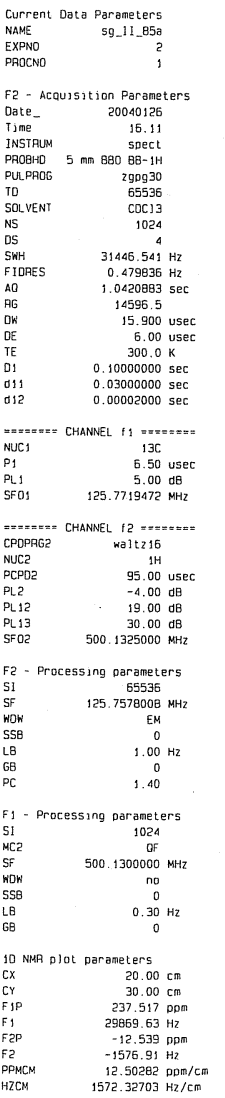



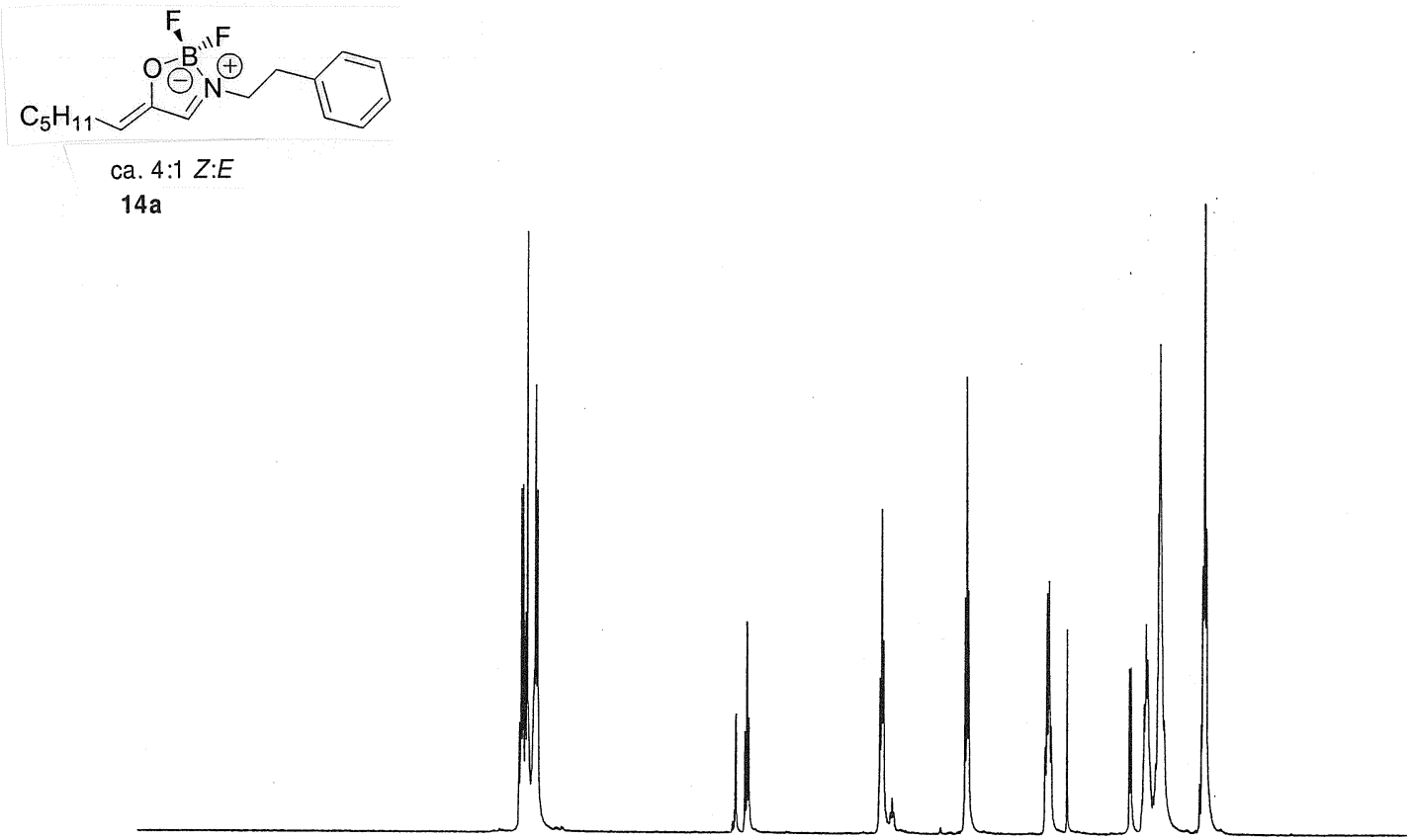

言

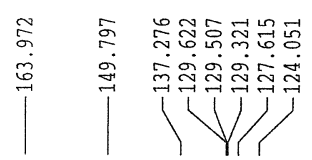

요욕ㅋ

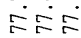

ind

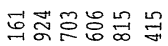

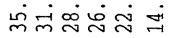

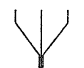

1111

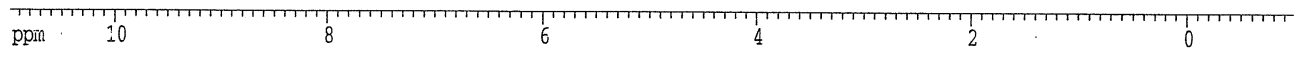

|
Current Data Parameters

NAME

PROCNO

F2 - Acquisition Parameters

Date_ 20040211

Time

5 PROBED 5 me multix400

PUPPOC 5 and Maltinel

SOLVENT

NS

$\quad 16$

$\begin{array}{ll}\text { SWH } & 4789.272 \mathrm{~Hz}\end{array}$

$\mathrm{AQ} \quad 3.4210291 \mathrm{sec}$

RG $\quad 143.7$

$\begin{array}{rr}104.400 \text { usec } \\ \mathrm{DE} & 4.50 \mathrm{usec}\end{array}$

$\begin{array}{lr}\mathrm{TE} & 300.0 \mathrm{~K} \\ \mathrm{D} 1 & 1.00000000 \mathrm{sec}\end{array}$

$==z==z=$ CHANNEL f1 $=z=z=z=$
NUC1 $1 \mathrm{H}$

$\begin{array}{lr}\text { NUC1 } & 1 \mathrm{H} \\ \text { P1 } & 7.70 \mathrm{use} \\ \text { PL1 } & -6.00 \mathrm{~dB}\end{array}$

SFO1 $\quad 400.1320007 \mathrm{MHz}$

F2 - Processing parameters

$\begin{array}{lr}\text { SI } & 16384 \\ \text { SI } & 400.1300000\end{array}$

$\begin{array}{lc}\text { WDW } & \text { EM } \\ \text { SSB } & 0 \\ \text { LB } & 0.30 \mathrm{~Hz} \\ \text { GB } & 0 \\ \text { PC } & 1.00\end{array}$

1D NMR plot parameters
CX $20.00 \mathrm{~cm}$

$\begin{array}{ll}\text { CX } & 20.00 \mathrm{~cm} \\ \text { CY } & 10.00 \mathrm{~cm}\end{array}$

$\begin{array}{ll}\text { F1P } & 10.985 \mathrm{pp}\end{array}$

$\begin{array}{lr}\text { F1 } & 4395.28 \mathrm{~Hz} \\ \text { F2P } & -0.985 \mathrm{ppm} \\ \text { F2 } & -393.99 \mathrm{~Hz}\end{array}$

PPMCM $\quad 0.59846 \mathrm{ppm} / \mathrm{cm}$

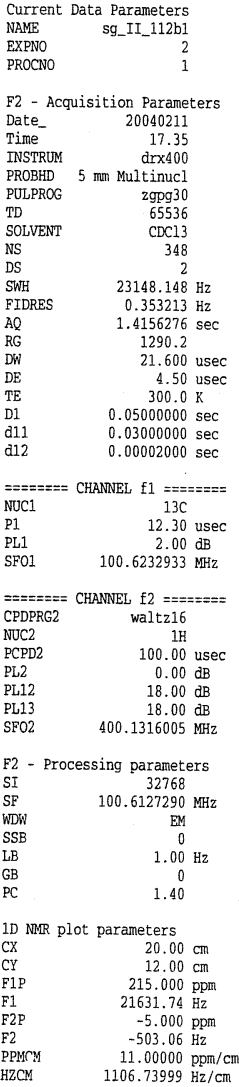

175

ppm $\quad 200$

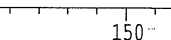

125

${ }_{10}^{10} S-57 \quad \frac{1}{75}$ 


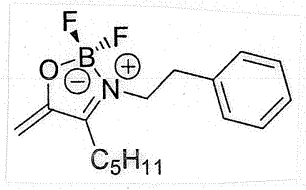

$14 b$

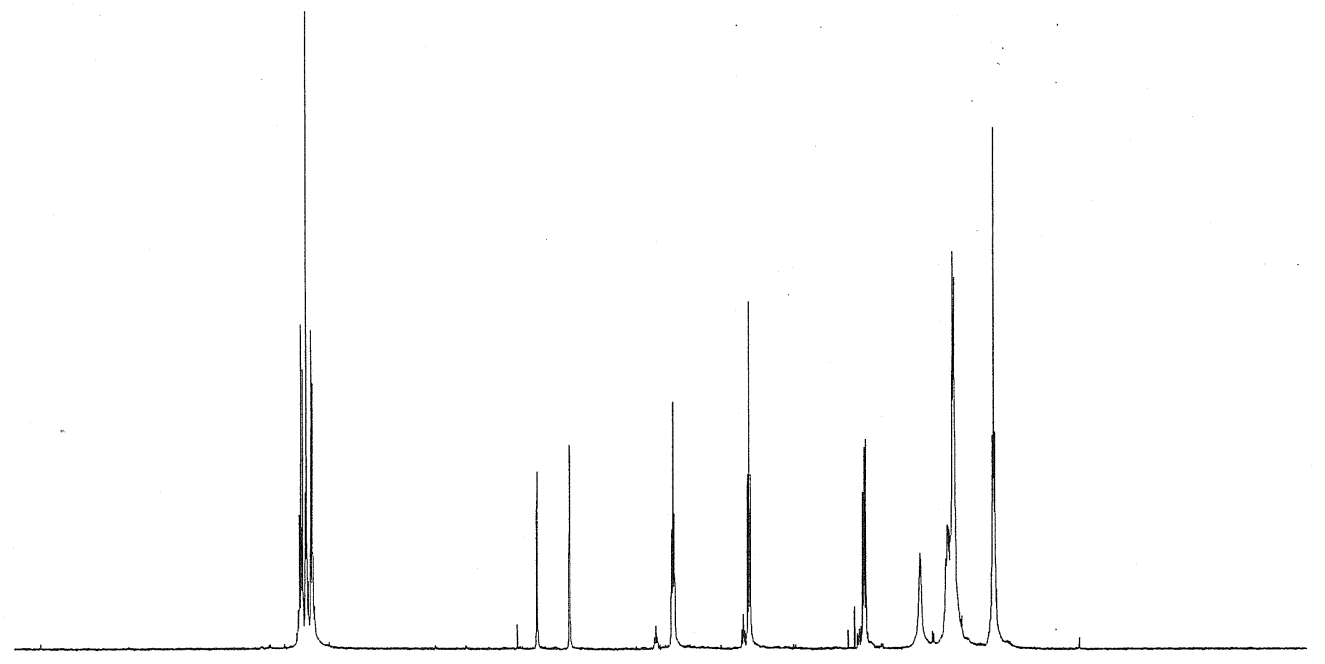

ppm

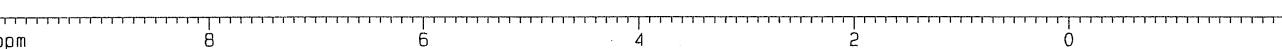

言
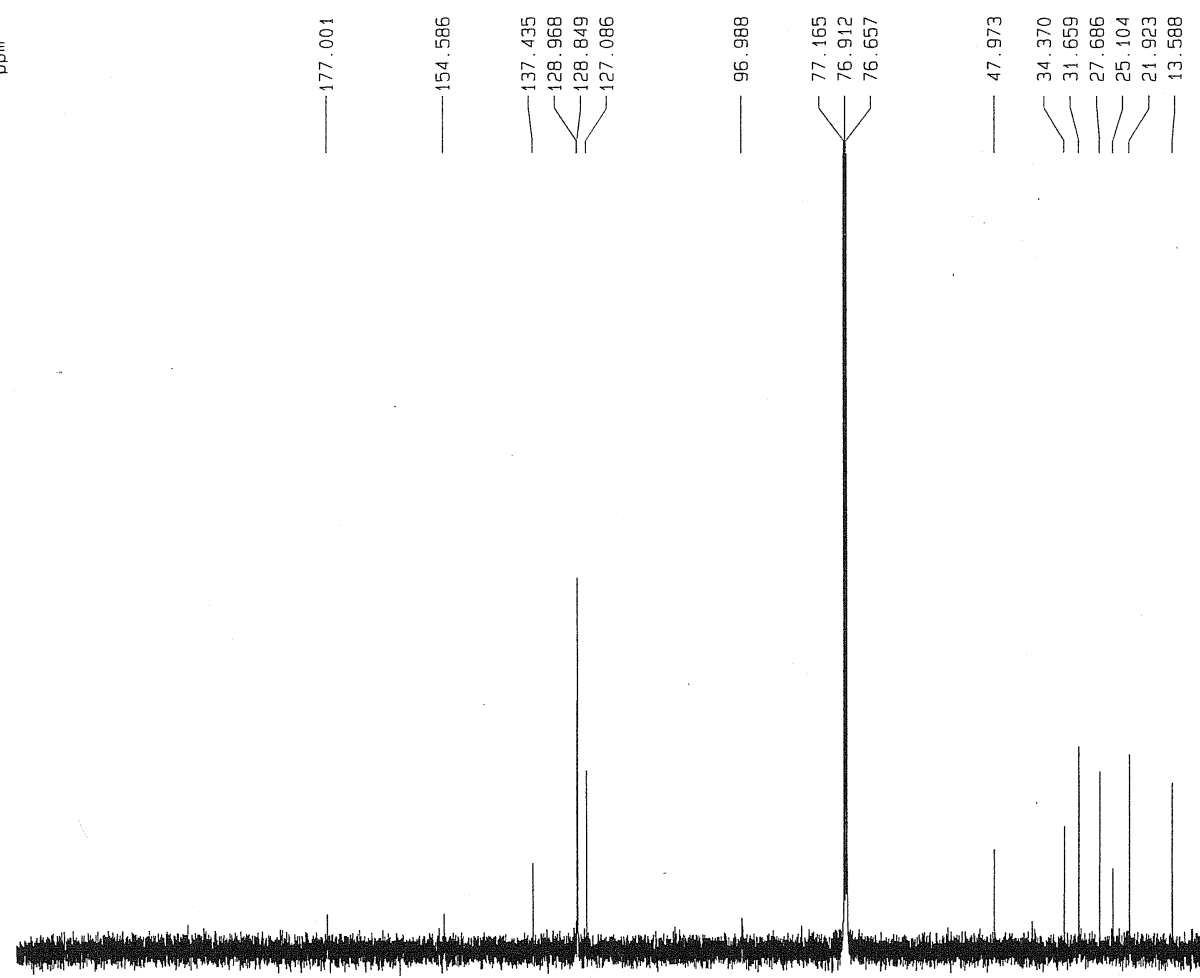

F2 - Acquisition Parameters

$\begin{array}{lr} & \\ \text { Date_ } & 20040226 \\ \text { Time } & 9.44\end{array}$

9.44
INSTRUM

РROBHD $5 \mathrm{~mm}$ вBO B8-1H

$\begin{array}{lr}\text { PULPROG } & 2930 \\ \text { TO } & 32768\end{array}$

SOLVENT

NS
DS
SWH

$\begin{array}{ll}\text { FIDAES } & 0.183399 \mathrm{~Hz} \\ \text { AQ } & 2.7264309 \mathrm{sec}\end{array}$

$\begin{array}{lr}\text { PG } & 645.1 \\ \text { OW } & 83.200 \text { usec }\end{array}$

3.200 usec
6.00 usec
$300.0 \mathrm{~K}$ $0.03000000 \mathrm{sec}$

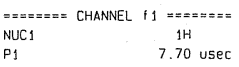

$\begin{array}{lr}\text { P1 } & 7.70 \text { usec } \\ \text { PL1 } & -4.00 \mathrm{~dB}\end{array}$

500.1320005 MHz

F2 - Processing parameters

$\begin{array}{lc}\text { SI } & 32768 \\ \text { SF } & 500.1320000 \mathrm{MHz} \\ \text { WOW } & \text { EM } \\ \text { SSB } & \text {. }\end{array}$

SSB

LB
GB
$P C$

F1 - Processing parameters
SI
1024.

$\begin{array}{lc}\text { SI } & 1024 \\ \text { MCZ } & \text { OF } \\ \text { SF } & 500.1300000 \mathrm{MHZ} \\ \text { WDW } & \text { OSINE }\end{array}$

SSE
LB
GB

$\begin{array}{lr}\text { 1D NMF plot parameters } \\ \text { CX } & 20.00 \mathrm{~cm} \\ \text { CY } & 10.00 \mathrm{~cm} \\ \text { F1P } & 10.008 \mathrm{ppm} \\ \text { F1 } & 5005.33 \mathrm{~Hz} \\ \text { F2P } & -2.008 \mathrm{ppm} \\ \text { F2 } & -1004.29 \mathrm{~Hz} \\ \text { PPMCM } & 0.60081 \mathrm{ppm} / \mathrm{cm} \\ \text { HZCM } & 300.48077 \mathrm{~Hz} / \mathrm{cm}\end{array}$

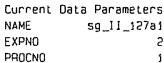

F2 - Acquisition Parameters

$\begin{array}{ll}\text { Date- } & 20040226 \\ \text { Time } & 9.54\end{array}$

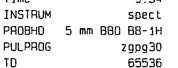

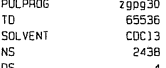

$\begin{array}{lr}\text { SWH } & 31446.541 \mathrm{~Hz} \\ \text { FIORES } & 0.479836 \mathrm{~Hz}\end{array}$

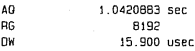

DE
TE
TE
D.

$0.03000000 \mathrm{sec}$

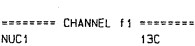

$\begin{array}{lr} & 13 \mathrm{C} \\ P_{1} & 6.50 \mathrm{usec} \\ P_{1} & 5.00 \mathrm{~dB} \\ \text { PL1 } & 125.7719972 \mathrm{MHz}\end{array}$

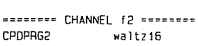

$\begin{array}{lc}\text { CPDPAG2 } & \text { waltz } 216 \\ \text { NUC2 } & \text { 1H } \\ \text { PPPO2 } & 95.00 \text { usec } \\ \text { PL2 } & -4.00 \mathrm{~dB} \\ \text { PL.12 } & 19.00 \mathrm{~dB} \\ \text { P.13 } & 30.00 \mathrm{~dB}\end{array}$

$\begin{array}{rl}30.00 \mathrm{~dB} \\ \mathrm{FF} 02 & 500.1325000 \mathrm{MH}\end{array}$

F2 - Processing parameters

$\begin{array}{lc}\text { SF } & 65536 \\ \text { SF } & 125.7578000 \mathrm{NH} \\ \text { HOW } & \text { EM } \\ \text { SSB } & 0\end{array}$

ᄂ

F1 - Processing parameters

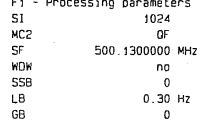

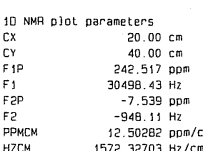

